Der Medizinischen Fakultät der Georg-August-Universität Göttingen eingereicht von Prof. Dr. med. U. Streeck, M.A.

\title{
Zur Wirksamkeit psychoanalytisch-interaktioneller Gruppentherapie in der stationären Allgemeinpsychiatrie
}

\author{
INAUGURAL-DISSERTATION \\ zur Erlangung des Doktorgrades \\ der Medizinischen Fakultät \\ der Georg-August-Universität zu Göttingen
}

vorgelegt von

Anke Valkyser, geb. Wolf

aus

Herne

Göttingen 2013 
Diese Dissertation wurde angefertigt in der Katholischen Krankenhaus GmbH Hagen und dem Asklepios Fachklinikum Göttingen in den Jahren 2007-2013 unter Anleitung von Prof. Dr. med. U. Streeck, M.A.

Dekan: Prof. Dr. rer. nat. H. K. Kroemer

I. Berichterstatter: Prof. Dr. med. U. Streeck, M.A.

II: Berichterstatter/in: Prof. Dr. med. Hermann-Lingen

III. Berichterstatter/in:

Tag der mündlichen Prüfung: 3.12.2013 


\section{Inhaltsverzeichnis}

Seite

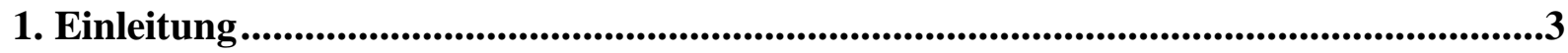

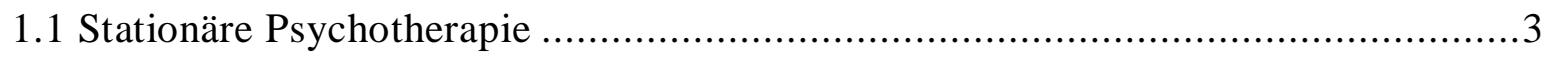

1.1.1 Historische Entwicklung in Deutschland .............................................. 3

1.1.2 Aktuelle Versorgungssituation stationärer Psychotherapie ..........................

1.2 Psychotherapie in der Psychiatrie ......................................................6

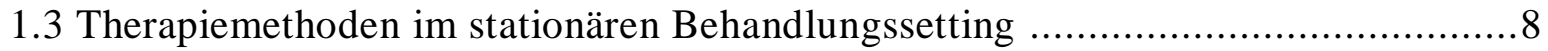

1.4 Psychoanalytisch-interaktionelle Therapie ............................................ 10

1.4.1 Psychoanalytische Behandlung struktureller Störungen ............................ 10

1.4.2 Psychoanalytisch-interaktionelle Gruppentherapie ................................. 15

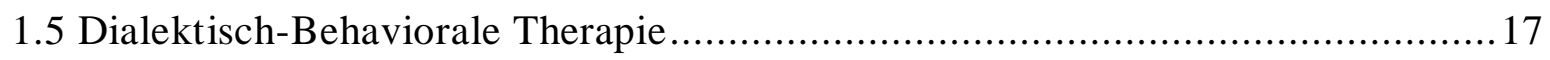

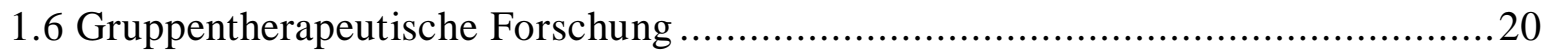

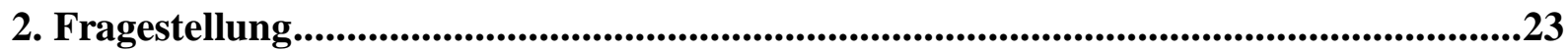

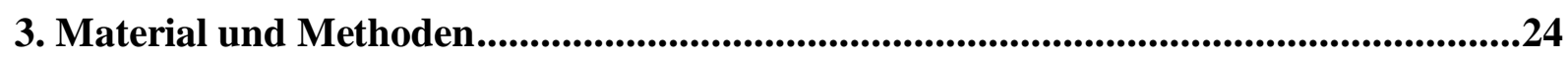

3.1 Die Patientenstichprobe .................................................................. 24

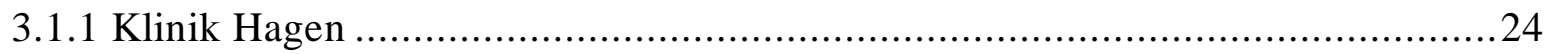

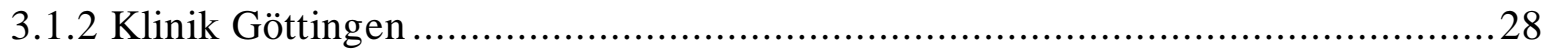

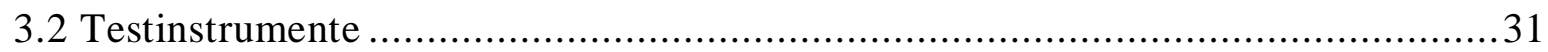

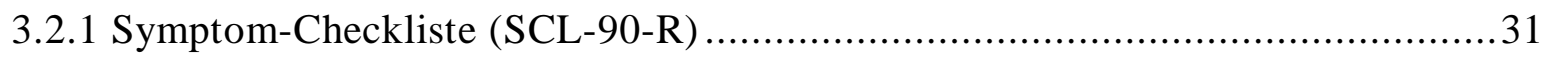

3.2.2 Inventar zur Erfassung interpersonaler Probleme (IIP) ............................... 32

3.2.3 Fragebogen zur Lebenszufriedenheit (FLZ) ............................................ 33

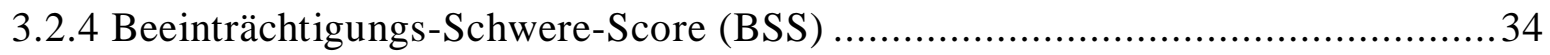

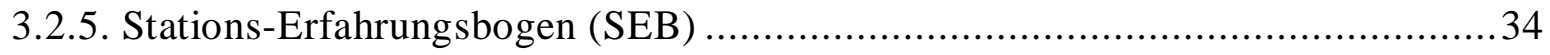

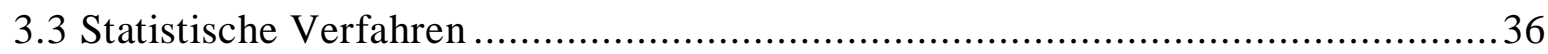

4. Ergebnisse ...........................................................................................................................................37

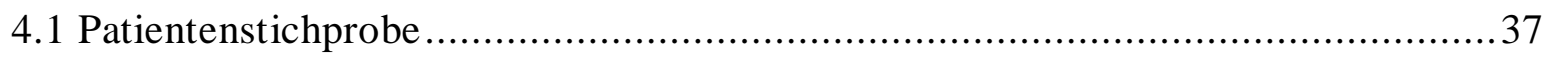

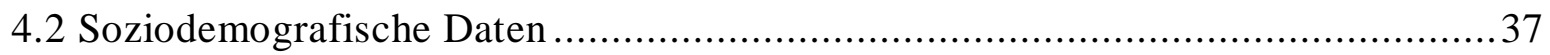

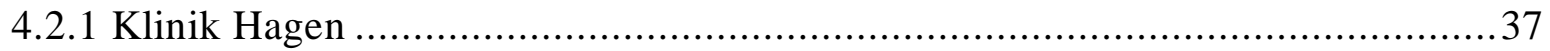

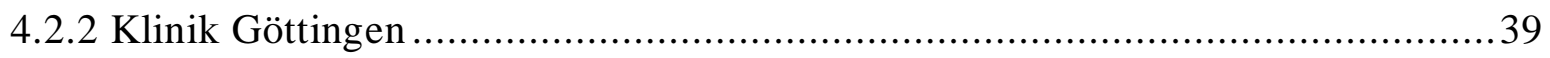

4.2.4 Vergleich der soziodemografischen Daten ............................................ 41 


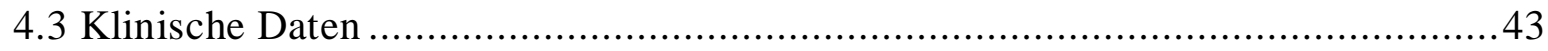

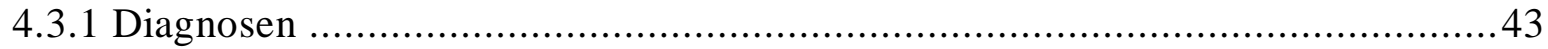

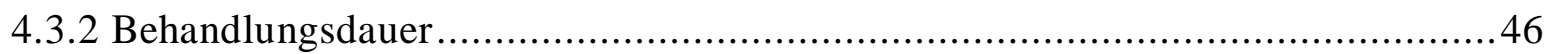

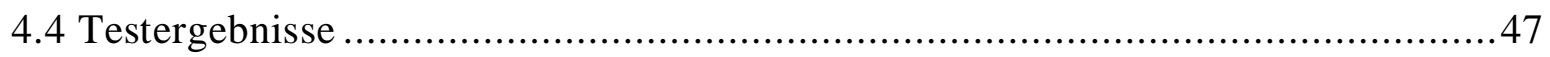

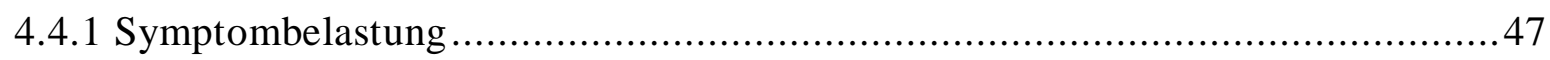

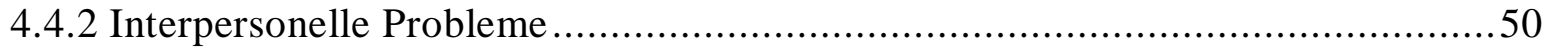

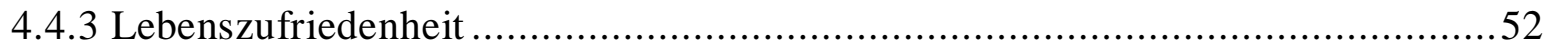

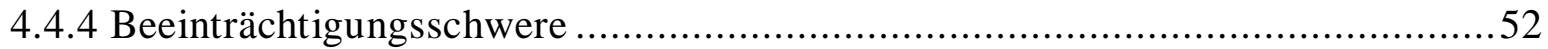

4.4.5 Einflussgrößen auf die Prä-Post-Testergebnisse ........................................54

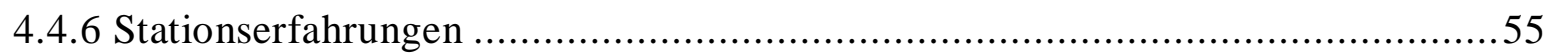

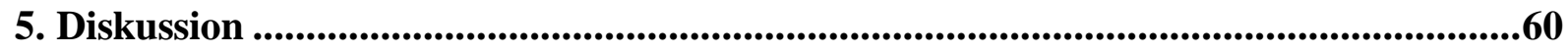

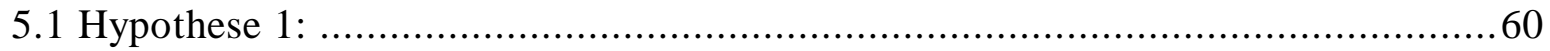

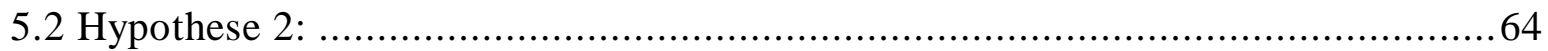

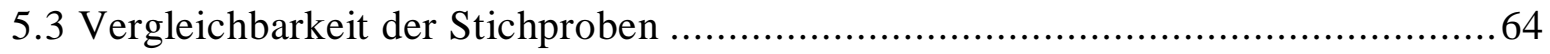

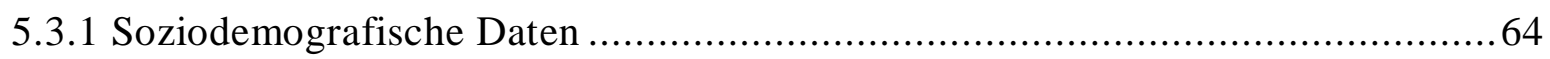

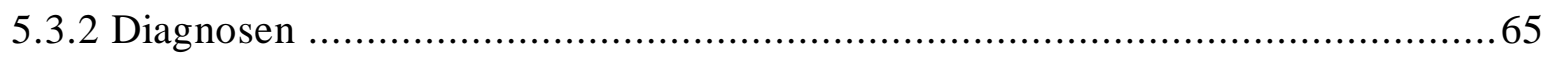

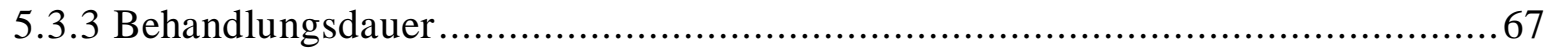

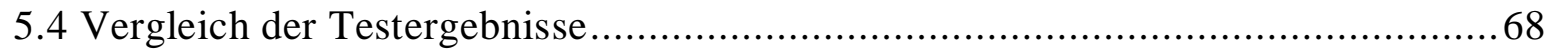

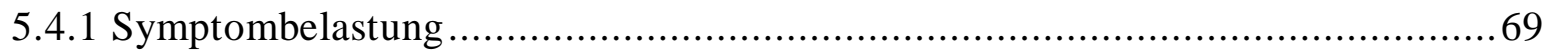

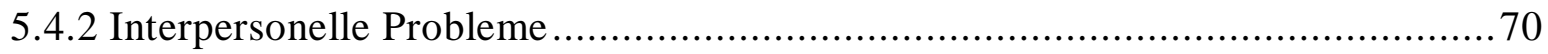

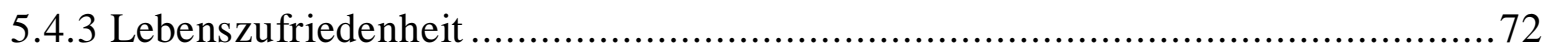

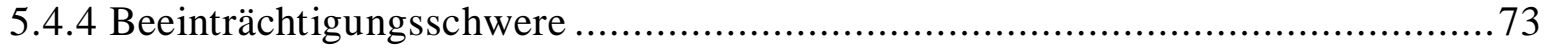

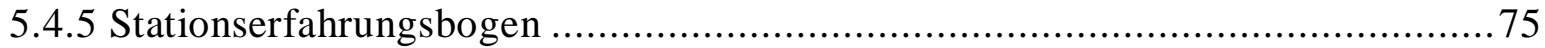

5.5 Einschränkungen und Ausblick .............................................................. 76

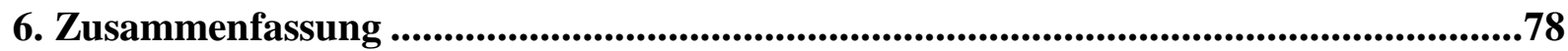

7. Literaturverzeichnis ....................................................................................................80 


\section{Einleitung}

\subsection{Stationäre Psychotherapie}

Psychotherapeutische Behandlungen nicht nur im ambulanten Einzelsetting, sondern auch als vollstationäre Therapie markieren eine Entwicklung, die sich vor allem in Deutschland etabliert hat. Dabei bezeichnet der Begriff der „stationären Psychotherapie“ keine einheitliche Methode und hat auch keinen einheitlichen historischen Ursprung. Dennoch gibt es Faktoren, die eine psychotherapeutische Behandlung im stationären Rahmen, unabhängig von der theoretischen Grundausrichtung der Therapiemethode, auszeichnen. Zum besseren Verständnis sei eine Darstellung der geschichtlichen Entwicklung vorangestellt.

\subsubsection{Historische Entwicklung in Deutschland}

Schepank (1988) gibt einen umfassenden Überblick über die historischen und gesellschaftspolitischen Hintergründe für die Entwicklung der stationären Psychotherapie in Deutschland. Ursachen für diese (im internationalen Vergleich spezielle) Entwicklung sind demnach vor allem finanzieller Wohlstand, verbunden mit einer durch die Sozialgesetzgebung geregelten Verteilung, die freiheitlich-demokratische Grundordnung und die Säkularisierung, ferner die Entwicklung einer psychoanalytisch orientierten Therapie neben der traditionellen psychiatrischen Theorie und Praxis.

Nach Senf (1988) zeigten sich bereits zur Anfangszeit zwei unterschiedliche Ansätze für die Etablierung psychotherapeutischer Verfahren im stationären Bereich, der anthropologische und der psychoanalytische. Der anthropologische Ansatz steht in enger Verbindung mit Viktor von Weizsäcker (1925) und der Integration psychotherapeutischer Aspekte in die somatische Medizin. So eröffnete von Weizsäcker im Jahre 1928 eine besondere „NeurotikerAbteilung“ als Teil der Nervenabteilung der Medizinischen Klinik der Universität Heidelberg. Der psychoanalytische Ansatz führte zur Gründung eigenständiger psychotherapeutischer Abteilungen oder Kliniken. Hier ist vor allem Ernst Simmel (1928) als maßgeblicher Entwickler eines psychoanalytischen Konzeptes zu nennen, der im Jahre 1927 die erste psychotherapeutische Klinik in Berlin-Tegel eröffnete.

Die klassische Psychiatrie nahm in Deutschland eine skeptische Haltung gegenüber der Psychoanalyse ein und verfolgte eine eher naturwissenschaftlich-biologistische Haltung. In den Jahren des Nationalsozialismus wurde diese aus ideologischen Gründen im Sinne der Konstitutionsbiologie weiter verschärft, die Psychoanalyse hingegen isoliert; sie geriet durch die Emigration psychoanalytisch tätiger $\ddot{A ̈ r t e}^{1}$ in dieser Zeit in Deutschland ins Abseits. Auch in den Jahren nach dem zweiten Weltkrieg blieb die Psychiatrie der Psychoanalyse gegenüber zunächst verschlossen (Schepank 1988).

\footnotetext{
${ }^{1}$ In dieser Arbeit wird allein aus Gründen der Übersichtlichkeit bei gemischtgeschlechtlichen Personengruppen die männliche Ausdrucksform verwendet. Dies stellt keine Bewertung dar.
} 
Eine zweite Phase der Entwicklung stationärer Psychotherapie schloss sich im Rahmen der Demokratisierung nach dem Ende des zweiten Weltkrieges an, in der einige psychoanalytische Kliniken gegründet wurden. So übernahm Gottfried Kühnel im Jahre 1949 das Landeskrankenhaus Tiefenbrunn bei Göttingen, das zur ersten psychotherapeutischen Großklinik wurde und bis heute besteht. Victor von Weizsäcker etablierte eine psychosomatische Abteilung an der Universitätsklinik Heidelberg, geleitet von Alexander Mitscherlich, um nur zwei Beispiele zu nennen. In den sechziger Jahren kam es zu einer zunehmenden Kooperation mit den Rentenversicherungen als Kostenträgern für ehemalige Kurbehandlungen, die nun in psychotherapeutische Heilbehandlungen umgewandelt wurden. Eine Vielzahl neu gegründeter psychotherapeutischer Kliniken war die Folge.

In den siebziger Jahren fanden einige tief greifende Änderungen in der psychiatrischen und psychotherapeutischen Versorgungslandschaft statt. So führte eine Reform der Ärztlichen Approbationsordnung mit der Erweiterung des Medizinstudiums um die Bereiche Psychosomatik, Psychotherapie, medizinische Psychologie und Soziologie zu der Gründung von Lehrstühlen für Psychosomatik an den Universitätskliniken. Des Weiteren wurde die Versorgung psychisch Kranker in Deutschland einer ausführlichen Erhebung unterzogen und mündete schließlich in die Psychiatrieenquete des Deutschen Bundestages (1975), die eine durchgreifende und nachhaltige Veränderung der psychiatrischen Versorgungsstruktur nach sich zog. Sie führte nicht nur zu einer Sektorisierung und wohnortnahen Versorgung der Bevölkerung durch psychiatrische Abteilungen und (verkleinerte) psychiatrische Fachkrankenhäuser, sondern auch zu einer stärkeren Integration psychotherapeutischer Methoden und sozialpsychiatrischer Ansätze in die psychiatrische Tätigkeit. Der Ausbau psychotherapeutischer Kliniken wurde gefördert, die Beschäftigung psychologischer Psychotherapeuten sowie anderer Spezialtherapeuten in psychiatrischen und psychotherapeutischen Kliniken ausgeweitet (Schepank 1988).

Bis in die siebziger Jahre war die stationäre Psychotherapie vorwiegend von der Psychoanalyse dominiert. Dies änderte sich, als die klinische Psychologie verhaltenstherapeutische Konzepte auch für den stationären Bereich entwickelte. Als erste verhaltenstherapeutische Klinik wurde Mitte der siebziger Jahre die Psychosomatische Klinik Windach gegründet, gefolgt von der Psychosomatischen Fachklinik Bad Dürkheim 1981 (Schepank 1988). Auch die Verhaltenstherapie nutzte hierbei die erweiterten Möglichkeiten therapeutischen Arbeitens im stationären Milieu, das den Patienten vielfältige korrigierende Erfahrungen ermöglicht. Die verhaltenstherapeutischen Methoden fanden in der folgenden Zeit zunehmend in der Psychiatrie und in der stationären Rehabilitationsbehandlung Anwendung (Kosarz und Schwarz 1988, Köllner und Vauth 2007).

\subsubsection{Aktuelle Versorgungssituation stationärer Psychotherapie}

Mittlerweile existiert ein differenziertes Versorgungsspektrum für stationäre psychotherapeutische Behandlung, ohne allerdings eine eindeutige Struktur aufzuweisen. In einer aktuelleren Untersuchung im Rahmen der psychosozialen Versorgungsforschung fanden 
Schulz et al. (2006) eine Dreiteilung der stationären psychotherapeutischen Versorgung in folgende Bereiche:

1. Kliniken für Psychiatrie und Psychotherapie

2. Kliniken für Psychosomatik und Psychotherapie

3. Psychosomatische Rehabilitation.

Sie stellen die Vorteile der Vielfalt der Versorgung in Frage, da „differenzielle Indikationskriterien nicht erkennbar und die Zuweisungsprozesse $\mathrm{zu}$ den verschiedenen Angeboten weitgehend nicht transparent sind." (zitiert nach Schulz et al. 2006, S.185). Dies ist eine immer noch fortgeführte Kontroverse (Braner et al. 2007).

Obwohl im Rahmen der Psychiatrieenquete (Deutscher Bundestag 1975) die wohnortnahe Versorgung durch psychiatrische Kliniken sichergestellt wurde, ist die psychotherapeutische Behandlung eher auf psychosomatische Abteilungen konzentriert. Insbesondere die Psychotherapieforschung findet überwiegend in psychosomatischen Fachkliniken statt (z. B. Strauß und Burgmeier-Lohse 1994, Hoffmann et al. 1998, Rudolf et al. 2004, Dinger und Schauenburg 2010), oder in psychiatrischen Universitätskliniken (Bohus et al. 2004, Kröger et al. 2006). Über die große Anzahl von Patienten, die in psychiatrischen Kliniken der Pflichtund Regelversorgung psychotherapeutisch behandelt werden, gibt es hingegen fast keine Forschungsergebnisse.

Aufgrund der Rahmenbedingungen weisen psychotherapeutische Behandlungen in psychiatrischen Kliniken besondere Merkmale auf, die sich auf Wirkfaktoren und Behandlungsergebnisse auswirken können, so dass sich Studienergebnisse nicht ohne Vorbehalt hierauf übertragen lassen. Diese Faktoren sind (zitiert nach Freyberger und Spitzer 2012, S.421):

- „ein breites diagnostisches Spektrum“

- „Patienten mit unzureichender eigener Behandlungsmotivation“

- „unterschiedliche Klinik- und Abteilungsstrukturen“, bezogen auf die Größe und die inhaltliche Ausrichtung

- „ein prozessuales Nebeneinander unterschiedlicher interner Settings“

- „kurze mittlere Behandlungszeiten (20-30 Tage)“

- „einen vergleichsweise inhomogenen Aus-, Fort- und Weiterbildungsstandard der beteiligten Mitarbeiter". 
Die vorliegende Untersuchung beschäftigt sich daher mit den Ergebnissen psychotherapeutischer Behandlung in einer psychiatrischen Klinik der Pflicht- und Regelversorgung, als Ergänzung zu den bestehenden Forschungsergebnissen zu stationärer Psychotherapie im psychosomatischen oder universitären Bereich.

\subsection{Psychotherapie in der Psychiatrie}

Die Psychiatrie in Deutschland hat (vorwiegend aus politisch-historischen Gründen, s.o.) eine kritische Haltung gegenüber der Psychotherapie über einen langen Zeitraum beibehalten und den Schwerpunkt eher auf die Behandlung schizophrener und affektiver Psychosen, mit einem biologistischen Ansatz, gelegt. Neurosen und andere psychische Störungen sind weniger beachtet worden. Dies zeigt sich auch daran, dass erst seit den 90er Jahren Psychotherapie ein zwingender Bestandteil des psychiatrischen Facharztes ist (Freyberger und Kächele 2010).

Psychodynamische Verfahren haben sich in der Psychiatrie in Deutschland sehr schwer getan (obwohl beispielsweise von Mentzos (2009) und Gabbard (2010) durchaus aktuelle deutschsprachige Lehrbücher für psychodynamische Therapie psychiatrischer Störungsbilder vorliegen); stattdessen haben sie ihren festen Platz in der Psychosomatik gefunden. In den USA, wo die Psychotherapie in die Psychiatrie integriert ist, sind hingegen psychodynamische Verfahren auch dort verbreitet, wobei eine lebendige Diskussion um deren Stellenwert besteht. Strupp (2001) wendet sich beispielsweise gegen die Vorherrschaft der empirisch belegten psychotherapeutischen Methoden, da er hierin ein $\mathrm{zu}$ sehr medizinisch betontes Verständnis sieht, Psychotherapie aber immer im Kontext einer einzigartigen therapeutischen Beziehung zu verstehen sei. Gabbard et al. (2002) sehen hingegen primär ungenügende Forschungsergebnisse als Grund für den abnehmenden Stellenwert psychoanalytischer Methoden und erachten es als notwendig, vermehrt kontrollierte Studien durchzuführen. Kernberg (2002) kritisiert den vermeintlichen ökonomischen Druck auf die psychodynamische Therapie, vor allem unter dem Gesichtspunkt der langen Behandlungszeit (ein Kritikpunkt, der auch in Deutschland diskutiert wird, in Vorbereitung der Einführung des DRG-Systems ${ }^{2}$ für psychiatrisch-psychotherapeutische Kliniken):

„Regarding economic pressures, the efforts to reduce payment for long-term psychotherapeutic treatment is part of the effort to control costs in psychiatry, which has already evinced its negative effects in the deterioration of psychiatric hospital practices in recent years, the typical chaotic psychopharmacological approaches to inadequately assessed patients resulting in multiple hospitalizations, and, probably, contributing to chronicity of illness in many cases.“ (zitiert nach Kernberg 2002, S. 498).

\footnotetext{
${ }^{2} \mathrm{DRG}=$ „Diagnosis Related Groups“ bezeichnet ein Vergütungssystem, das durch pauschale diagnosebezogene Vergütung in der stationären Behandlung charakterisiert ist und bisher nur in somatischen Fachgebieten angewendet wird.
} 
Weitere Argumente für den Verbleib psychoanalytischer Methoden in der Psychiatrie sind die strukturelle Veränderung als Behandlungsziel (die somit auch in Studien erfasst werden sollte, Wallerstein 2002) und die Wichtigkeit psychoanalytischen Verständnisses für intrapsychische Vorgänge bei psychiatrischen Krankheitsbildern, auch wenn diese nicht unbedingt primär psychoanalytisch behandelt werden (Auchincloss 2002).

Im Rahmen der Evidenzbasierten Medizin und der Entwicklung von Leitlinien ist die Psychotherapie in der Psychiatrie in Deutschland, auch für schwere psychische Störungsbilder, wieder in den Blickpunkt gerückt. So wird aktuell eine diagnoseübergreifende „S3-Leitlinie Psychosoziale Therapien bei schweren psychischen Störungen“ entwickelt (Riedel-Heller und Becker 2012), die sowohl soziotherapeutische und psychoedukative als auch verhaltenstherapeutische und psychodynamische Verfahren beinhaltet, die allerdings vor allem störungsspezifisch eingesetzt werden. Pfammatter und Junghan (2012) geben hierzu einen umfassenden Überblick, betonen aber auch die Bedeutung der therapeutischen Beziehung als allgemeinen Wirkfaktor jeglicher Art von Psychotherapie.

Es zeichnet sich demnach derzeit eine Entwicklung zu einer weniger schulen- als vielmehr störungsspezifischen Psychotherapie in der Psychiatrie ab. Hierbei sollen evidenzbasierte Therapieverfahren für das jeweilige Störungsbild zur Anwendung kommen. Berger und Hohagen (2012) bezeichnen diese Herangehensweise als störungsorientierte Psychotherapie und erläutern das Konzept wie folgt:

„Eine solche störungsspezifische Sichtweise bedeutet nicht, dass man die individuellen Erfordernisse und Bedürfnisse des Patienten außer Acht lässt. Das Konzept geht aber davon aus, dass Störungen zu einem Zustand führen, in dem Individuen mit prämorbid unterschiedlichen Charakterzügen und Eigenschaften durch die Erkrankung große Ähnlichkeiten im Verhalten, emotionalen Erleben und in kognitiven Abläufen aufweisen.“ (zitiert nach Berger und Hohagen 2012, S. 87)

Psychotherapie soll entsprechend den Empfehlungen einer von der Deutschen Gesellschaft für Psychiatrie, Psychotherapie und Nervenheilkunde (DGPPN) eingesetzten Expertenkommission künftig in einem modularen Ordnungssystem angeordnet werden, ähnlich der Vorgehensweise in der Dialektisch-Behavioralen Therapie. Es entsteht dabei ein klar strukturierter und standardisierter Algorithmus, der Therapeuten die Orientierung erleichtern soll und so z. B. die Wahl des Behandlungsfokus mit der geeigneten Intervention leichter verknüpfen lässt (Bohus et al. 2012). Eine völlige Abwendung von einer schulenbezogenen Ausbildung und eine Hinwendung zur Vermittlung schulenübergreifender psychotherapeutischer Basiskompetenzen, die stufenweise ausgebaut werden, sieht ein Modell zur künftigen psychotherapeutischen Weiterbildung in der Psychiatrie vor, wie es von Falkai und Herpertz (2012) vorgeschlagen wird.

In der vorliegenden Arbeit wird, in Abweichung von einem vorwiegend störungsspezifischen Ansatz untersucht, welche Wirkung ein psychodynamisches Therapieverfahren, die 
psychoanalytisch-interaktionelle Gruppentherapie, in einer eher störungsheterogenen Patientenpopulation (wie sie in psychiatrischen Akutversorgungskliniken in der Regel anzutreffen ist) haben kann.

\subsection{Therapiemethoden im stationären Behandlungssetting}

Methodisch war die stationäre Psychotherapie zu Beginn psychoanalytisch ausgerichtet, wurde aber schon bald durch die Arbeit von Bion (Bion 1961, Bion und Rickman 1943), der im Rahmen des sogenannten „Northfield-Experiments“ mit Kriegsneurotikern im Gruppenstatt im Einzelsetting arbeitete, und Main (1946) um das Konzept der therapeutischen Gemeinschaft erweitert. Die Situation des sozialen Miteinanders wird dabei genutzt, um den therapeutischen Prozess zu unterstützen.

Die Anwendung psychoanalytischer Arbeit in dem therapeutischen Raum der Station führte in der Folge zur Entwicklung unterschiedlicher Modelle. Im integrativen Modell (Janssen 1987), das sich in der psychodynamischen Therapie im Laufe der Zeit durchgesetzt hat, wird die gesamte Station zum Therapieraum; dabei werden im Sinne einer dynamischen Einheit (Hoffmann et al. 1981) Erlebens- und Verhaltensweisen des Patienten stets auf den therapeutischen Prozess auf der Station bezogen. Die stationäre Einheit mit ihren spezifischen Rahmenbedingungen wird hierbei gezielt genutzt, um die Übertragungsaufspaltung schwer gestörter Patienten, die im Kontakt mit den verschiedenen Stationsmitgliedern hervorgerufen werden, therapeutisch aufzugreifen und zusammenzuführen (Arfsten und Hoffmann 1978, Möhlen und Heising 1980, Janssen 1987). In diesem Modell werden somit psychoanalytische Therapiegrundsätze und soziale Klinikrealität integriert, was eine offene Kommunikation und Kooperation aller Teammitglieder voraussetzt.

Streeck (1998) betont den sozialen Kontext als bedeutenden Faktor für Erlebens- und Verhaltensweisen von Patienten, indem er darauf hinweist, dass Persönlichkeitsstörungen immer auch als Störungen des Sozialen $\mathrm{zu}$ verstehen sind und sich die frühen Beziehungserfahrungen vor allem im interpersonellen Handeln manifestieren. Er unterscheidet dabei eine vertikale Perspektive, die auf die intrapsychischen Vorgänge des Patienten abzielt, und eine horizontale Perspektive, die die interpersonellen Verhältnisse aller Beteiligten, Patienten und Mitarbeiter auf der Station, betrachtet. Beide Perspektiven sind nach seiner Ansicht eng miteinander verbunden und ermöglichen es in besonderer Weise, die innerseelischen Aspekte der Patienten und ihre Verbindung mit der sozialen Welt zu verstehen und therapeutisch zu bearbeiten. Er regt dabei an, den stationären Rahmen als „Spielraum“ im Sinne Winnicotts (1987) zu nutzen, in dem Erfahrungen in einem Übergangsraum zwischen innerer und äußerer Realität gemacht werden können. Dabei sollte die Behandlung an einem Fokus orientiert sein, auf den alle therapeutischen Maßnahmen hinarbeiten und hinter dem die Methodenzentrierung zurücksteht (Streeck 1991).

Basierend auf dem Konzept der therapeutischen Gemeinschaft und den klinischen Entwicklungen der stationären Psychotherapie postuliert Senf (2007) allgemeingültige Leitlinien, die sich im Rahmen des integrativen Modells stationärer Psychotherapie etabliert haben (zitiert nach Senf 2007, S.4): 
1. „Die Klinik ist das therapeutische Feld.“”

Stationäre Psychotherapie bedeutet Teilnahme an einer Gruppensituation und mindert die Gewichtung der therapeutischen Zweiersituation.

2. „Therapie findet durch eine Gruppe therapeutisch Tätiger statt.“:

Alle an der Behandlung beteiligten Therapeuten koordinieren die Behandlung miteinander. Wichtige Voraussetzung ist hierbei möglichst offene Kommunikation, Toleranz und die Kontinuität der Therapeutengruppe.

3. „Patienten inszenieren im therapeutischen Feld einer psychotherapeutischen Klinik ihre Konfliktpathologie":

Patienten setzen sich im sozialen Miteinander entsprechend ihrem innerseelischen Erleben in Beziehung und inszenieren ihre inneren Konflikte im interpersonellen Raum.

4. „Das therapeutische Potenzial und die Ressourcen der Patienten werden optimal gefördert.":

Die Patienten bringen sich selbstverantwortlich in die therapeutische Behandlung ein, setzen sich zu anderen in Beziehung und machen neue soziale Erfahrungen. Diese Erfahrungen werden in der therapeutischen Gemeinschaft reflektiert als Grundlage für soziales Lernen. Des Weiteren wird die äußere Lebenswelt der Patienten in die Therapie miteinbezogen.

Auch verhaltenstherapeutische Kliniken nutzen die Integration verschiedener Therapeuten und Therapiemethoden, legen den Schwerpunkt aber mehr auf störungsspezifische Behandlung. Zwei wesentliche Behandlungsbereiche sind einerseits die Beseitigung der spezifischen Beschwerden und dysfunktionaler Verhaltensweisen, andererseits die Aktivierung von Ressourcen. Die therapeutische Beziehung als eigenständiger therapierelevanter Aspekt spielt dabei nur eine untergeordnete Rolle bzw. gewann erst im Laufe der Zeit an Bedeutung (Kosarz und Schwarz 1988, Linehan 1996a). Der Einzeltherapeut hat hierbei die zentrale Funktion in der Planung und Durchführung der Therapie in Zusammenarbeit mit dem Patienten und einer co-therapeutischen pflegerischen Einzelbetreuung, Therapiebausteine werden nach indikativen, themenzentrierten Aspekten in die Behandlung einbezogen. Ein wichtiger Schwerpunkt liegt auf der Stärkung der Eigenverantwortlichkeit des Patienten im Umgang mit seinem Störungsbild (Borgart und Meermann 2004).

Die Kombination psychodynamischer und verhaltenstherapeutischer Methoden ist im stationären Bereich mittlerweile verbreitet und die Auswirkungen auf die Behandlungs- 
ergebnisse sind Gegenstand der Forschung (Hoffmann et al. 1998, Janssen et al. 1998, Geiser et al. 2004). Hoffmann et al. (1998) fanden z. B. deutlich bessere Ergebnisse bei agoraphoben Patienten, die an einer kombinierten psychodynamisch-verhaltenstherapeutischen Behandlung teilnahmen. Sie kamen zu sechs unterschiedlichen Hypothesen, die die Wechselwirkungen erklären könnten. Geiser et al. (2004) fanden nach Einführung eines verhaltenstherapeutischen Moduls in ein psychodynamisches Behandlungssetting eine Verbesserung der Symptomatik bei Angst- und Panikstörung, weisen aber auch darauf hin, dass sich der Widerstand gegen Bearbeitung zugrundeliegender psychodynamischer Konflikte erhöhte; somit kann auch eine Behinderung des Therapiefortschritts eintreten. Diese Studie ergänzt den Befund von Kordy et al. (1990), der besagt, dass die einzelnen Therapiekomponenten in ihrer subjektiven Bedeutung für den Patienten davon abhängen, in welchem Setting sie angeboten werden.

Neben psychodynamischen und verhaltenstherapeutischen Methoden werden auch andere Verfahren, die nicht der „Richtlinien-Psychotherapie“ im ambulanten Rahmen entsprechen, wie z. B. Hypno-, Gestalt- und Familientherapie, angewendet. Ein weiterer Schwerpunkt ist die Gruppentherapie. Stationäre Psychotherapie ermöglicht darüber hinaus die Integration non-verbaler ausdruckszentrierter und körpertherapeutischer Verfahren, insbesondere für die Behandlung schwerer gestörter Patienten. Was dabei für den Patienten nützlich ist, z. B. die Wahlmöglichkeiten für ihn hilfreicher Verfahren (Kordy et al. 1990) oder dass die Probleme im Beziehungsaufbau durch „Nebenbeziehungen“ abgefangen werden können (Bassler und Hoffmann 1993), erschwert andererseits die systematische Erforschung spezifischer Wirkfaktoren einzelner Therapieelemente, so auch der Gruppentherapie (vergl. auch Haubl 2012).

Auch in der vorliegenden Untersuchung wurden psychodynamische Verfahren, insbesondere in Form der psychoanalytisch-interaktionellen Gruppentherapie, und verhaltenstherapeutische Methoden, speziell Elemente der Dialektisch-Behavioralen Therapie, auf einer Station kombiniert. Diese Verfahren werden im Folgenden näher erörtert. Des Weiteren werden spezifisch gruppentherapeutische Wirkfaktoren und Forschungsergebnisse vorgestellt.

\subsection{Psychoanalytisch-interaktionelle Therapie}

\subsubsection{Psychoanalytische Behandlung struktureller Störungen}

Mit dem Ziel, psychoanalytische Methoden auch für die Behandlung schwerer gestörter Patienten, insbesondere Patienten, die an sogenannten strukturellen Störungen leiden, anwenden zu können, ist in der Klinik Tiefenbrunn bei Göttingen die psychoanalytischinteraktionelle Methode entwickelt worden (Heigl-Evers und Heigl 1983, Heigl-Evers und Streeck 1985, König K und Lindner 1992, Heigl-Evers und Ott 2002). Es handelt sich hierbei um Störungsbilder, die diagnostisch den schweren Persönlichkeitsstörungen zuzuordnen sind, d. h. in erster Linie Cluster-A- und -B-Persönlichkeitsstörungen nach DSM IV, Perversionen, Suchterkrankungen oder auch schwere psychosomatische Störungen. Da diese Patienten auf die Behandlung mit klassischer Psychoanalyse nicht oder mit Verschlechterung ihrer 
Symptome reagieren, stand die angewandte Psychoanalyse vor der Aufgabe, ihre Behandlungsvorstellungen $\mathrm{zu}$ modifizieren, „wenn die Psychoanalyse weiterhin eine therapeutische Methode von Relevanz bleiben soll“ (zitiert nach Fürstenau 1977, S.198, Hervorhebung entsprechend dem Original).

Worin bestehen die behandlungstechnischen Probleme? Die psychoanalytische Methode setzt das Vorhandensein eines „fiktiven Normal-Ich“ (Freud 1937/1961, Streeck 1983) voraus, dessen Funktionen durch einen neurotischen Konflikt außer Kraft gesetzt wurden (Konfliktpathologie). In der Behandlung soll durch Übertragungsprozesse auf den Psychoanalytiker gezielt eine partielle Regression herbeigeführt werden, um an unbewusstes Material zu gelangen und hierdurch unbewältigte (frühere) Entwicklungsprozesse zu bearbeiten. Dadurch soll der neurotische Konflikt behoben werden, damit die Ich-Funktionen wieder zur Verfügung stehen. Die zentrale Interventionsform ist dabei die Deutung, durch die der Therapeut dem Patienten mitteilt, welche unbewussten Motive sein Denken und Handeln beeinflussen.

Bei einer strukturellen Störung ist die Symptomatik hingegen nicht Ausdruck einer vorübergehenden „Lähmung“ der Ich-Funktionen, sondern ein Bewältigungsmechanismus ichstruktureller Defizite (Strukturpathologie): Werden durch regressionsfördernde Methoden, wie z. B. durch deutende Interventionen, die Ich-Funktionen noch weiter beeinträchtigt, kommt es zu einer bedrohlichen Labilisierung der psychischen Integrität mit fortschreitender Regression, impulshaftem Agieren, narzisstischem Rückzugsverhalten oder Wahnsymptomen (Fürstenau 1977, Heigl-Evers und Heigl 1983).

Defizite in den Ich-Funktionen betreffen die Fähigkeiten zur Selbstwahrnehmung und Selbststeuerung, der Objektwahrnehmung, Kommunikation, Realitätsprüfung und der Fähigkeit, Bindungen und Beziehungen flexibel zu gestalten. Es überwiegen interpersonale Abwehrfunktionen (Rudolf 2004), die sich in Teilobjektbeziehungen äußern. Andere Personen werden dabei nicht als eigenständige Individuen wahrgenommen, sondern in ihrer Funktion, die sie für die psychische Integrität des Patienten haben. So ist es den Patienten nicht möglich, im Rahmen einer psychoanalytischen Behandlung eine selbstreflexive Haltung einzunehmen beziehungsweise eine therapeutische Ich-Spaltung vorzunehmen. Vielmehr ist der Therapeut für die Aufrechterhaltung des seelischen Gleichgewichtes erforderlich und muss entsprechende Aufgaben in der Therapie übernehmen (Streeck 2007).

Die psychoanalytisch-interaktionelle Therapie wurde daher unter Einbeziehung ichpsychologischer und objektbeziehungs- und bindungstheoretischer Aspekte für die speziellen Bedürfnisse strukturell beeinträchtigter Patienten weiterentwickelt. Hierbei ist der Fokus nicht auf Unbewusstes und Regression, sondern auf (Vor-) Bewusstes und Progression ausgerichtet. Die Patienten sollen im Rahmen der Therapie ihre Defizite und deren Kompensation erkennen, mit den dazugehörigen interpersonellen Konsequenzen und Gefühlen, sowie Motivation und Geduld entwickeln, neue Fähigkeiten kennenzulernen und einzuüben (Heigl-Evers und Heigl 1983, Heigl-Evers und Streeck 1985).

Hierzu ist es erforderlich, dass der Therapeut eine aktive und personale Haltung einnimmt, sich als Gegenüber zur Verfügung stellt und zeitweise Hilfs-Ich-Funktionen übernimmt. Die 
zentrale Interventionsform ist das „Prinzip Antwort“. Die Art dieser Intervention und die Zielsetzung in der Therapie beschreiben Heigl-Evers und Henneberg-Mönch (1985) wie folgt:

„In der interaktionellen Methode stellt sich der Therapeut darauf ein, dem Patienten eine neue Art der Objektbeziehung anzubieten, indem er, orientiert an seinen Fremd- und Eigenwahrnehmungen, die er theoriegeleitet auswertet, dem Patienten auf dessen Äußerungen, seien sie verbaler oder averbaler Art oder auch in Handlung ausgedrückt, eine Antwort gibt, auf die der Patient seinerseits wiederum antworten kann. Die Antworten des Therapeuten sollen authentisch, hinsichtlich ihrer Äußerung jedoch selektiv sein, selektiv insofern, als der Therapeut sie dem jeweiligen Therapieziel entsprechend formuliert, authentisch insofern, als der Therapeut vor allem auch seine gefühlshaften inneren Antworten - immer unter dem Aspekt des Nutzens für den Patienten - in Worte faßt." (zitiert nach HeiglEvers und Henneberg-Mönch 1985, S.232)

Die Antwort des Therapeuten soll somit eine emotional authentische Reaktion zum Ausdruck bringen, gleichzeitig soll der Patient auf seine unter Umständen pathogene Objektwahrnehmung und hieraus entstehende interpersonelle Probleme hingewiesen werden. Heigl-Evers und Heigl (1983) empfehlen daher ein gut ausgewogenes Vorgehen:

„Eine solche interaktionelle Intervention oder ,Antwort' des Therapeuten sollte hinsichtlich ihres emotionalen Gehaltes immer eine Legierung von Libidinösem und Aggressivem sein, eine Mischung von Akzeptieren und Kritisieren. Gleichzeitig sollte das Angebot einer neuen Objektbeziehung gemacht werden, die zur Nachentwicklung defizitärer Ich-Funktionen anregt und die geeignet ist, im Patienten ein Gefühl der Hoffnung zu wecken und ihm eine Zukunftsperspektive zu eröffnen.“ (zitiert nach Heigl-Evers und Heigl 1983, S. 7)

Diese Interventionsform rückt unweigerlich stärker den interpersonellen Aspekt in den Vordergrund. Der Patient wird nicht vom Therapeuten „behandelt“, vielmehr vollzieht sich die Behandlung in der Interaktion. Dabei gibt es zwei Ebenen: die psychoanalytische, die unbewusste übertragungsrelevante Prozesse im Hintergrund wahrnimmt, und eine interaktionelle Ebene, die sich auf die interpersonellen Prozesse zwischen Patient und Therapeut bezieht (Berghaus 2005, Streeck 2007). Streeck (2002a) betont dabei, dass erst eine reale Objektbeziehung zum Therapeuten hergestellt werden muss, die tragfähig und verlässlich ist und um die sich der Therapeut gerade bei diesen Patienten aktiv bemühen muss. Eine gewisse Idealisierung des Therapeuten ist dabei erwünscht und sollte erst dann verändert werden, wenn andere beziehungsgestaltende Fähigkeiten entwickelt werden konnten. Durch den antwortenden Interventionsmodus bringt der Therapeut gleichwohl zum Ausdruck, dass er nicht nur verbunden, sondern auch getrennt vom Patienten ist, denn die Gefühle des Therapeuten ,sind allenfalls in Grenzen mit der Übertragungsrealität vereinbar, in deren 
Gewand der Patient ihn wahrnimmt“" (zitiert nach Streeck 2002a, S.127). Dabei ist unbedingt auf die Toleranzgrenzen des Patienten zu achten, damit die Beziehung erhalten bleibt.

Die therapeutische Haltung ist geprägt von einem hohen Maß an Authentizität und wacher Präsenz, einer unbedingten Akzeptanz. Durch die emotionale Antwort, haltende Funktion und die Übernahme von Hilfs-Ich-Funktionen soll es dem Patienten schrittweise ermöglicht werden, eigene emotionale Zustände zu erkennen und zu regulieren, aber auch die Motive und Emotionen seines Gegenübers einschätzen zu lernen und in Verbindung mit dessen Verhalten zu bringen. Diese Prozesse kommen im Konzept der „Mentalisierung“ (Berghaus 2005, Fonagy und Bateman 2006, Allen und Fonagy 2009) zum Ausdruck und haben in den letzten Jahren zunehmend Bedeutung erlangt.

Der interpersonelle Aspekt, die Art und Weise zu kommunizieren (und zwar sowohl sprachlich als auch nicht-sprachlich), wird von Streeck (2002b, 2007) als wesentlich hervorgehoben. Er weist darauf hin, dass sich die Kommunikation zwischen Patient und Therapeut immer im Kontext der Situation vollzieht und sprachliche und nicht-sprachliche Kommunikation dabei untrennbar miteinander verbunden sind. Sowohl Patient als auch Therapeut verhalten sich in bestimmter Weise zueinander in der Therapie, so dass auch im therapeutischen Dialog nicht in erster Linie zählt, was gesagt wird, sondern wie es gesagt wird. Gerade Mimik und Gestik spielen hierbei eine wichtige Rolle für das Zustandekommen und die Gestaltung der therapeutischen Beziehung.

Hier werden die beiden Dimensionen der psychoanalytisch-interaktionellen Therapie nochmals besonders deutlich. Gerade Patienten mit schweren Persönlichkeitsstörungen weisen in ihrer Biografie in der Regel schwere Beziehungstraumatisierungen auf. Da diese Traumatisierungen häufig bereits früh aufgetreten sind, sind sie dem sprachlichen, deklarativen Gedächtnis nicht zugänglich, sondern im prozeduralen, körperlichen Gedächtnis gespeichert. Diese Erfahrungen werden folglich durch das Verhalten mitgeteilt, das somit eine biografische, vertikale Achse und, da sich dieses Verhalten im Kontext der aktuellen Situation abspielt, eine gegenwärtige, horizontale Dimension enthält (Streeck 2007, siehe hierzu auch Abbildung 1). 


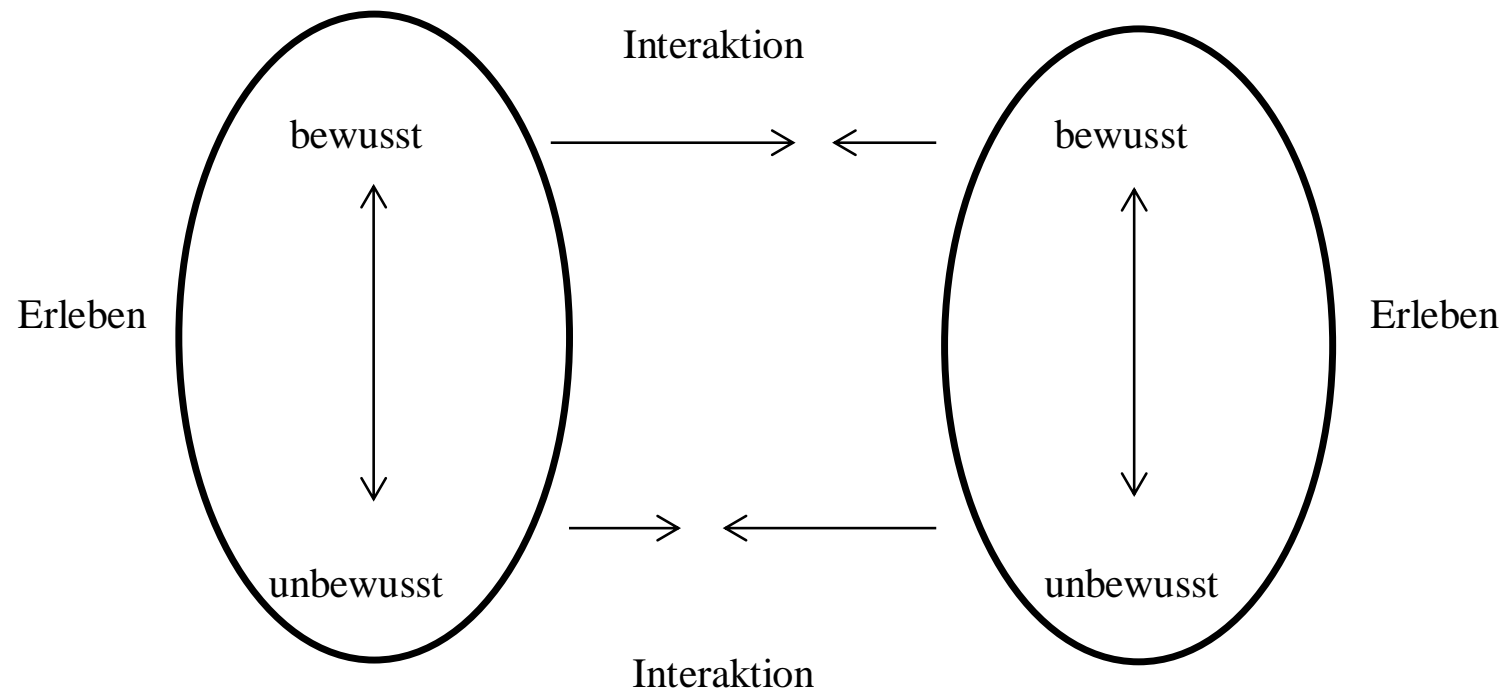

Patient

Therapeut

Abb. 1: Vertikale und horizontale Ebene des Erlebens und Verhaltens in der PatientTherapeut-Interaktion, modifiziert nach Streeck (2007, S. 88) 


\subsubsection{Psychoanalytisch-interaktionelle Gruppentherapie}

Da es sich bei Persönlichkeitsstörungen bzw. ichstrukturellen Defiziten in erster Linie um Beziehungsstörungen handelt, liegt es nahe, diese gruppentherapeutisch zu behandeln. Der Vorteil liegt dabei nicht nur darin, dass sich die Beziehungsmuster in ihren verschiedenen Facetten im Mehrpersonenkontext deutlicher zeigen und untersucht werden können. Wie oben bereits erläutert wurde, sind Patienten mit solchen Störungsbildern in der Regel nicht in der Lage, gleichzeitig die dyadische Beziehung im Einzelkontakt mit dem Therapeuten zu gestalten und darüber zu reflektieren. In der Gruppe unter mehreren Personen kann der Therapeut hingegen diese reflexive Funktion übernehmen und mit den Patienten gemeinsam die interpersonellen Beziehungen und deren Beeinträchtigungen zum Gegenstand der Untersuchung machen (Ott 2001, Streeck 2002c).

Auch hier gilt, dass die Beziehungen in der Gruppe einerseits durch biografische Erfahrungen der einzelnen Gruppenmitglieder und deren Objektrepräsentanzen geprägt sind, andererseits im aktuellen Kontext der Gruppensituation, die ,geregelt“ und bewältigt werden muss, zu verstehen sind. Die Beziehungsgestaltung in der Gruppe entsteht also ebenfalls an der Schnittstelle zwischen vertikalen früheren Beziehungserfahrungen und horizontalen, aktuellen Beziehungen. Dabei wird „Interpersonelles Verhalten (...) nicht als individuelles Merkmal behandelt; vielmehr wird das Verhalten des einzelnen Patienten in der Gruppe immer im Kontext des Verhaltens Anderer verstanden“ (zitiert nach Streeck 2002c, S. 114, Hervorhebung entsprechend Original).

Die diagnostische Aufmerksamkeit des Therapeuten richtet sich dabei auf die Gestaltung der Gruppensituation, die sich meist an vergleichbaren realen Situationen orientiert (z. B. „Schulstunde“ oder „Kaffeeklatsch“), und die bereits implizite Normen für den Umgang miteinander und die Rollenverteilung innerhalb der Gruppe enthält. Vor allem nichtsprachliche Signale wie Schweigen oder Kopfnicken steuern dabei erwünschte und nichterwünschte Verhaltensweisen. Es ergeben sich hieraus wichtige Hinweise für die aktuell aktivierten Objektbeziehungsmuster. Die geltenden Normen regulieren dabei Nähe und Distanz, Ausdruck von Affekten und die Art und Weise, wie mit unterschiedlichen Bedürfnissen in der Gruppe verfahren wird. Neben der Diagnostik der Beziehungen in der Gruppe gilt die Aufmerksamkeit des Therapeuten auch den einzelnen Gruppenmitgliedern, indem er auf deren Schwierigkeiten und Fertigkeiten, entstehende oder abgewehrte Affekte, Selbst- Objektdifferenzierung und überwiegende Objektbeziehungen achtet, Zusammenhänge zwischen intrapsychischen und interpersonellen Faktoren herstellt und die Patienten hierauf aufmerksam macht.

Aus dem oben Gesagten ergibt sich, dass die psychoanalytisch-interaktionelle Gruppentherapie eine Therapie des Einzelnen im Kontakt und durch den Kontakt mit anderen in der Gruppe ist. Dies funktioniert nur dann, wenn sich die Patienten in der Gruppe möglichst „natürlich“ und alltagsnah zueinander verhalten. Daher ist eine gewisse Störungsheterogenität auch eher als günstig anzusehen. Die Alltagsnähe der Gruppen soll durch die Vorgabe der freien Interaktion unter Beachtung der Belastbarkeitsgrenzen der einzelnen Gruppenmitglieder gefördert werden, was zuvor in einem Vorgespräch mit dem Therapeuten vereinbart wird. 
Die zentrale Interventionstechnik ist auch hier die selektiv-authentische Antwort des Therapeuten, indem er äußert, welche Gefühle oder Handlungsimpulse sich bei ihm als Reaktion auf die Geschehnisse in der Gruppe oder gegenüber einzelnen Gruppenmitgliedern einstellen. Hiermit nimmt er implizit auf die geltenden Normen Einfluss und gibt sich als Person zu erkennen, die sich durch andere berühren lässt, aber gleichzeitig eigene Grenzen hat und diese zum Ausdruck bringt. Falls erforderlich übernimmt der Therapeut Hilfs-IchFunktionen, indem er beispielsweise darauf hinweist, wie er sich in bestimmten Situationen verhalten würde oder aktiv progressivere Normen in der Gruppe etabliert. Darüber hinaus kann der Therapeut Erklärungen $\mathrm{zu}$ beobachteten Verhaltensweisen und deren Zusammenhang mit interpersonellen Beziehungen abgeben (Streeck 2002c).

Nach den Untersuchungen von Davies-Osterkamp et al. (1992) werden von den Patienten besonders die Erfahrungen, anderen ähnlich $\mathrm{zu}$ sein oder sich $\mathrm{zu}$ unterscheiden, sowie entsprechende Rückmeldungen sowohl als besonders hilfreich, aber auch als schädlich eingestuft. Es scheint daher eine besonders wichtige Aufgabe des Therapeuten zu sein, bei Rückmeldungen regulierend im Hinblick auf die Einhaltung der Toleranzgrenzen einzugreifen. Vom Therapeuten wiederum wird vor allem eine spezifische „Antwort“ als hilfreich erlebt, nämlich diejenige, in der er mitteilt, wie er sich in bestimmten Situationen fühlen oder verhalten würde, also indem er eine Hilfs-Ich-Funktion übernimmt, die nicht zu offen normierend eingreift.

Die Haltung des Therapeuten wird, wie in der Einzeltherapie, durch vier wesentliche Aspekte geprägt: Wache Präsenz, Respekt gegenüber dem Schicksal anderer, unbedingte emotionale Akzeptanz und ein hohes Maß an Authentizität eigenen Gefühlen gegenüber, auch wenn diese schwer auszuhalten sind, verbunden mit der Bereitschaft, diese dosiert mitzuteilen (Ott 2001, Streeck 2002c).

Als allgemeines Ziel der psychoanalytisch-interaktionellen Gruppentherapie kann formuliert werden, „dass an die Stelle wiederkehrender dysfunktionaler interpersoneller Verhaltensmuster größere innere und interpersonelle Autonomie tritt“. Die Patienten erhalten so die Gelegenheit ,sich des eigenen Erlebens und Verhaltens im Kontext des Erlebens und Verhaltens Anderer sicherer zu werden und sich unter Wahrung der eigenen Individualität im Kontakt mit Anderen zu bewegen und damit bessere Verhältnisse zu schaffen“ (beide Zitate Streeck 2002c, S. 125).

Die psychoanalytisch-interaktionelle Gruppentherapie hat als eine Form des sogenannten „Göttinger Modells" für die Behandlung in Gruppen mit psychoanalytischen Methoden vor allem in Deutschland weite Verbreitung gefunden. Zum Einsatz kommt die Methode natürlich bei der Behandlung von Persönlichkeitsstörungen (Davies-Osterkamp et al. 1992, Konzag und Fikentscher 1998, Rabung et al. 2005, Leichsenring et al. 2007), wobei hier gute Wirksamkeitsnachweise erbracht werden konnten. Weitere Einsatzgebiete der psychoanalytisch-interaktionellen Gruppentherapie ergeben sich in der Suchtbehandlung (Gantner 1999), als geschlossene Kurzgruppen in der Rehabilitationsbehandlung (Günther und Lindner 1999), in der Behandlung somatoformer Schmerzstörungen (Nickel und Egle 1999) und auch bei der Behandlung traumatisierter Patienten (Schellenberg et al. 2004). In etwas modifizierter Form, unter stärkerer Betonung der Hilfs-Ich-Funktionen mit stärkerer 
Binnenstruktur, sieht Bolm (2005) auch ein Einsatzgebiet in der Akutpsychiatrie; dies wird unterstützt auch durch die positiven Ergebnisse von Leichsenring et al. (2005) in der Behandlung von Patienten mit Erkrankungen aus dem schizophrenen Formenkreis.

\subsection{Dialektisch-Behaviorale Therapie}

Über einen langen Zeitraum war die Behandlung von strukturellen Persönlichkeitsstörungen Schwerpunkt psychodynamischer Psychotherapie. Dies hat sich im Verlauf der letzten 20 Jahre geändert, als sich die Dialektisch-Behaviorale Therapie (DBT; Linehan 1996a), eine verhaltenstherapeutische Methode zur Behandlung der Borderline-Persönlichkeitsstörung, recht rasch etabliert hat.

In der Arbeit mit chronisch parasuizidalen Borderline-Patientinnen hat sich Linehan in den 80er Jahren intensiv mit wirksamen Techniken für diese schwierig $\mathrm{zu}$ behandelnde Patientengruppe beschäftigt. So ließ sie ihre Therapiesitzungen von ihrem Therapeutenteam regelmäßig beobachten, um herauszufinden, welche Interventionen als besonders hilfreich anzusehen sind. Es zeigte sich, dass neben klassisch kognitiv-verhaltenstherapeutischen Techniken andere Faktoren wie Ermutigung, Akzeptanz (im Gegensatz zu andauerndem Streben nach Veränderung) und ein hohes Maß an Flexibilität des Therapeuten hilfreich sind. Basierend auf diesen Erfahrungen, dem biosozialen Modell zur Entstehung der BorderlinePersönlichkeitsstörung und einer dialektischen Weltsicht entwickelte sie die DialektischBehaviorale Therapiemethode.

In der dialektischen Sichtweise stehen individuelle Verhaltensweisen und Umwelt in reziproker Beziehung zueinander und beeinflussen sich wechselseitig. Biologische und Umgebungseinflüsse werden als gleichberechtigt angesehen. Die Betonung liegt auf einer ganzheitlichen Sicht der Realität. Gleichzeitig bewegt sich die Realität zwischen zwei entgegengesetzten Kräften, die als „These“ und „Antithese“ bezeichnet werden, deren Integration zur „Synthese“ zu einer neuen Entwicklungsstufe führt, woraufhin dieser Vorgang sich wiederholt.

Das biosoziale Modell geht bei der Borderline-Störung von einer biologisch begründeten Fehlfunktion der Emotionsregulation aus, die durch invalidierende Umweltfaktoren zusätzlich verstärkt wird. Das Kind lernt somit nicht, seinen Gefühlen zu vertrauen und diese zu regulieren, vielmehr wird durch invalidierende Umweltbedingungen das Fühlen und Bewerten von Situationen als „falsch“ erlernt. In der weiteren Entwicklung als Erwachsene neigen Borderline-Patienten somit dazu, eigene Gefühle $\mathrm{zu}$ entwerten und erleben sich als grundsätzlich insuffizient. Bereits kleine Situationen des Scheiterns führen zu Selbsthass und massiven negativen Gefühlsreaktionen, die häufig wiederum an starke Schamgefühle geknüpft sind, da sie gelernt haben, dass emotionale Berührtheit und Verletzbarkeit etwas „nicht Richtiges“ sind. Gleichzeitig reagieren diese Patienten aufgrund ihrer biologisch begründeten Emotionsregulationsstörung extrem, was einen „circulus vitiosus“ initiiert. Daher werden negative Emotionen in der Folge möglichst gemieden, da sie für unaushaltbar gehalten werden, was wiederum neue Lernerfahrungen behindert. 
Linehan (1996a) interpretiert daher die Problematik von Borderline-Patienten als Scheitern an der Dialektik, da sie in ihrer Wahrnehmung auf dichotome Denkmuster eingeengt sind, ähnlich dem Prinzip der „Spaltung“ aus psychoanalytischer Sicht. In ihrer Welt gibt es lediglich ein Verharren in „These“ oder „Antithese“, die „Synthese“" scheint ihnen unmöglich zu sein. Ein anhaltender innerer Konflikt ist die Folge.

Die therapeutische Arbeit orientiert sich daher mehr an der dialektischen Haltung und unterscheidet sich in folgenden Punkten von der veränderungsorientierten „klassischen“ Verhaltenstherapie (zitiert nach Linehan 1996a):

„(1) Die Betonung von Akzeptanz und Validierung des Verhaltens, so wie es momentan auftritt; (2) die schwerpunktmäßige Behandlung von Verhaltensweisen, die die Therapie gefährden; (3) die Betonung der grundlegenden Wichtigkeit der therapeutischen Beziehung für die Behandlung; und (4) die Betonung von dialektischen Prozessen“(S. 16).

Die DBT ist modular angeordnet und primär für eine ambulante Behandlung von einem bis zwei Jahren Dauer konzipiert (Linehan et al. 1991, Bohus 2002). Die vier Module bestehen aus

- Einzeltherapie

- Telefonberatung

- Fertigkeitentraining (Skills) in der Gruppe

- Supervision für den Therapeuten.

Die Behandlung folgt einer hierarchisierten Struktur der Behandlungsziele. In der ersten Phase erfolgen zunächst Informationen über das Störungsbild und die Methode, Ziele werden formuliert und ein Behandlungsvertrag geschlossen. Die nächste Ebene befasst sich vorrangig mit Problemen auf der Verhaltensebene, die das Leben oder die Therapie bedrohen. Nachfolgend sind die Behandlung von Traumafolgen und Verhaltensweisen, die die Lebensqualität beeinträchtigen. Der Inhalt der Therapiesitzungen richtet sich danach, auf welcher Ebene sich Therapeut und Patient befinden, Probleme auf einer höherrangigen Ebene werden immer zuerst bearbeitet (Bohus 2002).

Eine dialektische Haltung zwischen Veränderung (insbesondere in Bezug auf extreme emotionsgebundene Verhaltensweisen) und Akzeptanz (eigener Gefühle, Wahrnehmungen und Bedürfnisse) durchdringt das gesamte Behandlungsmanagement. Ein wesentlicher Aspekt ist dabei die Vermittlung spezieller Fertigkeiten (Skills) in der Gruppe. Diese Gruppen sind manualgeleitet und bestehen wiederum aus vier Modulen (Linehan 1996b, Bohus 2002): 
- Stresstoleranz:

Selbstmanagement starker Emotionen und Anspannungszustände

- Umgang mit Gefühlen:

Identifikation, Regulierung und Distanzierung von (unangenehmen) Gefühlen

- zwischenmenschliche Fertigkeiten:

Förderung sozialer Kompetenz unter Beachtung emotionaler und kognitiver Einflüsse

- Achtsamkeit:

Bewertungsfreies Beobachten innerer und äußerer Prozesse in Anlehnung an ZenMeditation.

Nachdem sich gute Behandlungserfolge in der ambulanten Behandlung gezeigt haben, lag es nahe, die DBT auch für stationäre Behandlungen $\mathrm{zu}$ modifizieren. Barley et al. (1993) beschreiben die Einführung dieser Methode in ein vormals psychodynamisches Behandlungskonzept mit gutem Erfolg und Reduktion insbesondere parasuizidaler Handlungen. Sie betonen, dass dieser Ansatz gut mit dem psychodynamischen Verständnis von Persönlichkeitsstörungen übereinstimmt und wegen seines gut verständlichen Konzeptes von Patienten und Personal gut angenommen wurde. In Deutschland haben Bohus und Haaf (2001) ein stationäres Behandlungskonzept entworfen, das sich auf eine dreimonatige Behandlungszeit bezieht. Die Therapieziele sind hierbei vor allem die Bewältigung suizidaler Verhaltensweisen, Verstärkung von Therapiecompliance gegenüber therapiebehindernden Verhaltensweisen und die Entwicklung von Fertigkeiten, die eine ambulante Weiterbehandlung ermöglichen. Einzeltherapeut, Pflegekräfte und andere Therapeuten arbeiten möglichst transparent in enger Kooperation mit den Patienten zusammen. Dabei werden die Patienten angehalten, sich auch im dialektischen Sinn gegenseitig zu unterstützen. Eine Adaptation der Skillsgruppen für den stationären Bereich beschreiben Stiglmayr et al. (2002).

Als zentraler Aspekt genießt das Skillstraining in der DBT besondere Aufmerksamkeit. Dieses Training wird in der Gruppe durchgeführt. Im Gegensatz zu psychodynamischen Gruppentherapien handelt es sich hierbei allerdings um eine psychoedukative Gruppe mit reinem „Teaching“-Charakter, um das Gruppenklima zu entspannen. Gruppendynamische Faktoren werden nur insofern genutzt, als dass sich die Patienten gegenseitig unterstützen, gemeinsam Lösungen erarbeiten und so stützende Funktionen ausüben. Diese Form der Gruppentherapie im Sinne einer stationären Kurzgruppentherapie ist von Springer und Silk (1996) kritisch diskutiert worden. Die Förderung der Kohäsion und positiver Übertragung auf den Therapeuten, das Wecken von Hoffnung werden als durchaus förderlich hierbei gesehen. Kritikpunkte betreffen die bewusste Vermeidung aggressiver Gefühle, deren Bearbeitung möglicherweise einen wichtigen therapeutischen Stellenwert hat, und die Verhinderung tieferer Einsichtsprozesse in das Selbst.

Ob das Training der Fertigkeiten auch ohne die Einbettung in ein DBT-Behandlungskonzept gut wirksam ist, lässt sich bisher nicht empirisch belegen (Stiglmayr et al. 2002). Linehan 
(1996a) selbst sieht dies kritisch, da der Einzeltherapeut in der DBT wichtige Verstärkerfunktionen zur Integration der Fertigkeiten übernimmt. Arbeitet dieser hingegen mit einer anderen Methode, könnte dies zur Verwirrung der Patienten führen. Einen positiven Effekt ambulanter Skills-Gruppentherapie für Borderline-Patienten (ohne begleitende DBTBehandlung) im Vergleich mit psychodynamisch orientierter „Standardgruppentherapie“ berichten demgegenüber Soler et al. (2009). In einer Untersuchung mit chronisch depressiven Patienten zeigte sich ebenfalls ein signifikant größerer Behandlungserfolg durch Skillsgruppenbehandlung im Vergleich zu einer Kontrollgruppe unter Wartelistenbedingung (Harley et al. 2008), allerdings war die Stichprobe mit 19 Teilnehmern nur recht klein.

Für die DBT gibt es mittlerweile zahlreiche überzeugende Wirkungsnachweise für die Behandlung von Borderline-Patienten, insbesondere was die Reduktion suizidaler und parasuizidaler Verhaltensweisen, Selbstschädigungen und die Notwendigkeit stationärer Behandlung betrifft, dabei zeigt sich durchgehend eine niedrigere Abbrecherquote als in Kontrollgruppen (Linehan et al. 1991, Linehan et al. 1999, Turner 2000, Koons et al. 2001, Linehan et al. 2006; s. auch in der Übersicht von Lynch et al. 2007) Ein positiver Einfluss auf interpersonale Schwierigkeiten ist ebenfalls nachweisbar (Linehan et al. 1994). Die DBT gilt somit derzeit als die am besten evaluierte Behandlungsmethode für BorderlinePersönlichkeitsstörungen (Bohus und Kröger 2011).

Auch für andere Störungsbilder gibt es Wirksamkeitshinweise, insbesondere für komorbiden Suchtmittelgebrauch (Linehan et al. 1999, Harned et al. 2008). Eine Wirksamkeit für Depressionen, insbesondere durch Fertigkeitentraining (Harley et al. 2008), und bei chronisch depressiven älteren Erwachsenen mit komorbider Persönlichkeitsstörung sowie in der Behandlung von Essstörungen zeichnet sich ab (Lynch et al. 2007), bedarf aber weiterer Forschung. Harned et al. (2008) fanden hingegen keinen Unterschied in der Wirksamkeit auf komorbide Angststörungen, Essstörungen oder schwere depressive Störungen gegenüber einer Kontrollgruppe.

In der stationären Behandlung von Borderline-Patienten in Deutschland durch DBT zeigen sich überzeugende Behandlungserfolge, auch was die Verbesserung komorbider Achse-IStörungen angeht (Bohus et al. 2000, Bohus et al. 2004, Kröger et al. 2006).

\subsection{Gruppentherapeutische Forschung}

Gruppentherapie wird in der stationären Behandlung sowohl psychodynamisch als auch verhaltenstherapeutisch orientiert durchgeführt. Psychodynamische Verfahren fokussieren dabei mehr auf gruppendynamische und interpersonelle Aspekte, während verhaltenstherapeutische Gruppen eher höher strukturiert und leiterzentriert ablaufen, ohne die therapeutische Funktion der Gruppe an sich zu betonen (König O 2012). So ist das interpersonelle Geschehen ein wesentlicher Faktor in der psychoanalytisch-interaktionellen Gruppentherapie; in den Skills-Gruppen der Dialektisch-Behavioralen Therapie werden hingegen interpersonelle Aspekte bewusst zugunsten strukturierter Informationsvermittlung und gemeinsamer Übungen im Hintergrund gehalten (s.o.). Weitere verhaltenstherapeutische Gruppen, die häufig gerade in der Psychiatrie eingesetzt werden, sind z. B. die 
psychoedukativen Gruppen, die störungsspezifische Informationsvermittlung zum Inhalt haben.

Im Vergleich zur Einzeltherapie weist insbesondere psychodynamische Gruppentherapie spezifische Wirkfaktoren auf, die sich nur in der Mehrpersonenbeziehung entwickeln können, wie Tschuschke (2004) betont: „Es ist kein Wirkfaktor einzelpsychotherapeutischer Settings bekannt, der nicht auch in therapeutischen Gruppensettings zum Tragen kommen könnte. Hingegen gibt es mehrere gruppenspezifische Wirkfaktoren, die im Einzelsetting aufgrund der dyadischen Situation nicht auftreten können“ (zitiert nach Tschuschke 2004, S. 104). Yalom (1996) hat sich mit diesen Wirkfaktoren ausführlich beschäftigt und identifizierte elf unterschiedliche Primärfaktoren (zitiert nach Yalom 1996, S. 21):

1. „Hoffnung-Einflößen“

2. „Universalität des Leidens“

3. „Mitteilung von Informationen“

4. „Altruismus“

5. „Korrigierende Rekapitulation der primären Familiengruppe“

6. „Entwicklung von Techniken des mitmenschlichen Umgangs“

7. „Nachahmendes Verhalten“

8. „Interpersonales Lernen“

9. „Gruppenkohäsion“

10. „Katharsis“

11. „Existenzielle Faktoren“.

Insbesondere die Bedeutung der Gruppenkohäsion wird in gruppentherapeutischer Forschung immer wieder hervorgehoben. Eine gute Gruppenkohäsion wird dabei als günstige Voraussetzung für einen Behandlungserfolg gewertet (Tschuschke et al. 1992, Marziali et al. 1997, Kirchmann et al. 2009, Dinger und Schauenburg 2010), teilweise aber auch schon als therapeutischer Erfolg im Sinne einer Wendung interpersoneller zu intrapersonaler Abwehr (Grabhorn et al. 2002). Eine Abnahme der Kohäsion kann im Verlauf ebenfalls günstig sein im Sinne eines zunehmenden Individuationsprozesses (Tschuschke et al. 1992) oder bei Patienten, deren Interaktionsstil als ,zu freundlich“ gekennzeichnet ist (Dinger und Schauenburg 2010).

Gruppentherapeutische Forschung im stationären Rahmen findet nach einer Metaanalyse von Kösters und Strauß (2007) überwiegend in Deutschland statt. Dabei gibt es auch hier einen deutlichen Trend zu störungsspezifischen Gruppentherapieverfahren und eine Abwendung von störungsheterogenen Gruppen. Psychodynamische Gruppentherapien werden vor allem in der Prozessforschung und weniger in der Wirksamkeitsforschung berücksichtigt (Burlingame et al. 2002). Auf die Probleme zur Qualitätssicherung stationärer Gruppentherapie weisen Strauß et al. (1998) hin und regen an, z. B. durch den gezielten Einsatz von Gruppentherapiefragebögen spezifische gruppentherapeutische Aspekte abbilden zu können. 
Die vorliegende Studie soll einen Beitrag dazu leisten, den Einfluss psychoanalytischinteraktioneller Gruppentherapie (als einem psychodynamischen Gruppenverfahren) auf die Behandlung heterogener Störungsbilder in der stationären Psychotherapie in der Psychiatrie zu evaluieren. 


\section{Fragestellung}

Die psychotherapeutische Behandlung von psychiatrischen Patienten findet häufig wohnortnah in allgemeinpsychiatrischen Kliniken statt, ohne dass die Wirksamkeit Gegenstand der Forschung ist. Im Laufe der vergangenen Jahre haben sich im allgemeinpsychiatrischen Bereich eher störungsspezifische, auf verhaltenstherapeutischer Grundlage basierende Behandlungsverfahren etabliert. Über die Wirkung psychodynamischer psychotherapeutischer Behandlungen in allgemeinpsychiatrischen Kliniken (ohne universitären Hintergrund) ist dagegen wenig bekannt. Die psychoanalytisch-interaktionelle Gruppentherapie bietet dabei eine Behandlungsmethode auf psychodynamischer Grundlage, die seit langem auch für psychiatrische Patienten mit schweren Persönlichkeitsstörungen und gelegentlich auch schizophrenen Psychosen erfolgreich eingesetzt wird; allerdings liegen bisher keine Studien zur spezifischen Wirksamkeit dieser Gruppentherapiemethode in einer allgemeinpsychiatrischen Akutklinik vor.

Die Studie soll daher die Wirksamkeit der psychoanalytisch-interaktionellen Gruppentherapie in einer psychiatrischen Abteilung einer Klinik der Pflicht- und Regelversorgung untersuchen. Insbesondere soll geprüft werden, ob sich die Behandlungsergebnisse unterscheiden von den Ergebnissen der Behandlung einer Kontrollgruppe, die mit Dialektisch-Behavioraler Therapie (DBT) behandelt wurde.

Hierzu lassen sich zwei Hypothesen formulieren:

Hypothese 1: „Es wird angenommen, dass Patienten, die auf einer allgemeinpsychiatrischen Station auf psychodynamischer Grundlage mit psychoanalytisch-interaktioneller Gruppentherapie und Dialektisch-Behavioralen Therapiebausteinen behandelt werden, Verbesserungen ihrer Symptomatik im Verlauf der Behandlung aufweisen.“

Hypothese 2: „Es wird angenommen, dass Patienten, die mit Dialektisch-Behavioraler Therapie allein behandelt werden, ebenfalls Verbesserungen bezïglich ihrer Symptome zeigen, sich diese Behandlungsergebnisse aber unterscheiden von denen der Patienten, die zusätzlich an psychoanalytisch-interaktioneller Gruppentherapie teilnehmen.“ 


\section{Material und Methoden}

\subsection{Die Patientenstichprobe}

Für die vorliegende Arbeit wurden in den Jahren 2007 bis 2009 in der Klinik für Psychiatrie und Psychotherapie der Katholischen Krankenhaus GmbH Hagen und der Klinik für Psychiatrie und Psychotherapie des Asklepios Fachklinikums Göttingen (vormals Niedersächsisches Landeskrankenhaus Göttingen) jeweils 40 Patientinnen und Patienten zu Beginn, während und am Ende einer stationären Psychotherapie im Hinblick auf die Veränderung der Symptomatik und ihre Erfahrungen auf der Station während der Behandlung untersucht. Die Teilnahme an der Studie war freiwillig. Die Patienten gingen unter einem von ihnen frei gewählten Codenamen in die Studie ein, so dass die Datenauswertung anonymisiert war. Die Datenerhebung wurde beendet, als mindestens 40 vollständige Datensätze vorlagen. Patienten, die die Behandlung vorzeitig beendeten und die Abschlussuntersuchung nicht durchführten, wurden nicht in die Studie einbezogen. Neben den Testergebnissen wurden allgemeine soziodemografische Daten wie Alter, Geschlecht und Schulabschluss sowie die Diagnose nach ICD 10 (Dilling et al. 2006) und die stationäre Behandlungsdauer erhoben.

Die Patientengruppen unterschieden sich in ihrer Behandlung entsprechend der Behandlungskonzepte der teilnehmenden Kliniken. Patienten, die in der Klinik Hagen behandelt wurden, nahmen an einem psychodynamischen Behandlungskonzept mit einem Schwerpunkt auf psychoanalytisch-interaktioneller Gruppentherapie (Heigl-Evers und Ott 2002) teil, ergänzt durch Elemente der Dialektisch-Behavioralen Therapie (Linehan 1996a). Die Patientengruppe in Göttingen erhielt eine Behandlung nach den Grundlagen der Dialektisch-Behavioralen Therapie (Linehan 1996a), ohne psychoanalytisch-interaktionelle Gruppentherapie.

Die Charakteristika der einzelnen Kliniken werden im Folgenden beschrieben.

\subsubsection{Klinik Hagen}

Die Klinik für Psychiatrie und Psychotherapie ist eine Abteilungspsychiatrie an einem großen Allgemeinkrankenhaus, der Katholischen Krankenhaus $\mathrm{GmbH}$, die sich über drei Häuser im Stadtgebiet von Hagen verteilt. Die Abteilung befindet sich im St. Johannes Hospital, im Stadtteil Hagen-Boele.

Die Klinik umfasst 105 stationäre und 25 tagesklinische Behandlungsplätze und ist für die psychiatrische Pflicht- und Regelversorgung der Stadt Hagen mit ca. 190000 Einwohnern zuständig. Die stationäre Behandlung findet auf einer fakultativ geschlossenen, zwei offenen allgemeinpsychiatrischen Stationen, einer geronto-psychiatrischen sowie einer Psychotherapiestation statt und umfasst somit das gesamte Behandlungsspektrum psychiatrischer Erkrankungen, mit Ausnahme primärer Suchterkrankungen und Essstörungen. 
Die ambulante Weiterbehandlung besonders schwer erkrankter Patienten wird über eine angeschlossene Institutsambulanz gewährleistet.

Die Psychotherapiestation, Station 15, hat 16 stationäre Behandlungsplätze. Das Behandlungsangebot richtet sich schwerpunktmäßig an Patienten mit Depressionen, Ängsten und Persönlichkeitsstörungen, auch mit komorbidem Suchtmittelgebrauch, sofern dieser nicht im Vordergrund steht. Die Aufnahme erfolgt in der Regel als Akutaufnahme oder elektiv als Zuweisung durch einen niedergelassenen Facharzt, oder als interne Verlegung von einer der psychiatrischen Stationen, wenn eine psychotherapeutische Behandlung indiziert erscheint. Dabei ist die Aufnahmeindikation bewusst mit einer niedrigen Schwelle angelegt und breit gefasst, um den Bedürfnissen einer wohnortnahen Akutversorgungsklinik Rechnung zu tragen. Hierdurch bedingt finden, insofern die Umstände es erfordern, auch Patienten mit akuten Lebenskrisen oder mit einer akuten Psychose auf dieser Station Aufnahme. Ein Vorgespräch ist für die Aufnahme nicht zwingend erforderlich und findet eher selten statt.

An die Aufnahme schließt sich (ausgenommen sind akute psychiatrische Kriseninterventionen) zunächst eine ca. zweiwöchige Diagnosephase an. Hierbei erfolgt in den therapeutischen Einzelgesprächen und einer oberärztlichen Zweitsicht die Diagnostik, sowohl in psychiatrischer Hinsicht nach den Kriterien des ICD 10 (Dilling et al. 2006) als auch unter psychodynamischen und ichstrukturellen Gesichtspunkten. Anschließend werden individuelle Therapieziele festgelegt.

Wird die Indikation für eine psychotherapeutische Behandlung gestellt, wechseln die Patienten in die Psychotherapiegruppe. Besteht keine Indikation für eine psychotherapeutische Behandlung oder sind die Patienten nicht ausreichend stabil für eine gruppentherapeutische Behandlung, nehmen sie an einem eher supportiv-strukturierenden Behandlungsprogramm teil. In die Studie wurden nur solche Patienten eingeschlossen, die in der Psychotherapiegruppe behandelt wurden.

Die Behandlung ist psychodynamisch ausgerichtet und wird durch verhaltenstherapeutische Elemente, insbesondere der Dialektisch-Behavioralen Therapie, ergänzt.

Das Behandlungsteam auf der Station setzt sich aus verschiedenen Berufsgruppen zusammen. Die Abstimmung der psychotherapeutischen Arbeit erfolgt in regelmäßigen Teambesprechungen. Die Basis der psychotherapeutischen Behandlung sind dabei regelmäßige therapeutische Einzelgespräche, für 30 bis 50 Minuten einmal wöchentlich, und die Teilnahme an der psychoanalytisch-interaktionellen Gruppentherapie dreimal wöchentlich für 60 Minuten. Die Pflegemitarbeiter arbeiten im Bezugspflegesystem und sind langjährig erfahren in psychoanalytisch-interaktioneller Arbeit, teilweise auch geschult in DialektischBehavioraler Therapie. Ergänzend nehmen die Patienten an den komplementären handlungsorientierten Therapien wie Ergotherapie und Tanztherapie teil, je nach Indikation auch an Kraftsport oder Entspannungstraining. Für die Klärung sozialer Schwierigkeiten im Alltag finden Beratungsgespräche mit der Sozialarbeiterin statt. Bei Bedarf werden auch Angehörige in Form von Paar- oder Familiengesprächen in die Behandlung miteinbezogen.

Die therapeutischen Einzelgespräche werden durch eine Psychologische Psychotherapeutin, mit abgeschlossener verhaltenstherapeutischer Ausbildung, und einen Assistenzarzt in 
Weiterbildung zum Facharzt für Psychiatrie und Psychotherapie, entsprechend der Weiterbildungsermächtigung des leitenden Arztes, geführt. Hierbei finden wöchentliche oberärztliche Supervisionen statt.

Die psychoanalytisch-interaktionelle Gruppentherapie wird von der Oberärztin geleitet, die eine Ausbildung in dieser Therapieform bei der Arbeitsgemeinschaft für die Anwendung der Psychoanalyse in Gruppen e.V. in Göttingen abgeschlossen hat. Begleitend fanden während der gesamten Studiendauer regelmäßige Supervisionen der Gruppenbehandlungen $\operatorname{statt}^{3}$.

Patienten, die an ausgeprägter Anspannung leiden und Schwierigkeiten bei der Impulskontrolle haben, nehmen an einer durch die Psychologin geleiteten Skillsgruppe teil sowie an einer durch die Patienten in Eigenverantwortung durchgeführten SkillsÜbungsgruppe. Die Skillsgruppe wird entsprechend der Vorgaben der DialektischBehavioralen Therapie (Linehan 1996a) durchgeführt und wurde von der Psychologin bei der Arbeitsgemeinschaft Wissenschaftliche Psychotherapie in Freiburg erlernt. Ferner wird eine Achtsamkeitsgruppe, die vom Pflegepersonal der Station durchgeführt wird, angeboten. Für Verhaltensweisen, die die therapeutische Zusammenarbeit gefährden oder im therapeutischen Fokus als veränderungswert eingeschätzt wurden, fertigen die Patienten eine Verhaltensanalyse an, die im Einzelgespräch im Hinblick auf die Entwicklung von alternativen Bewältigungsstrategien durchgearbeitet wird.

Entsprechend der Heterogenität der Störungsbilder und den geltenden Behandlungsleitlinien der Psychiatrie wird auch eine medikamentöse Behandlung begleitend zur psychotherapeutischen Behandlung durchgeführt. Den größten Anteil hat hierbei der Einsatz von Antidepressiva. Bei psychosenahen oder psychotischen Zuständen sowie anhaltenden Flash-backs und Dissoziationen werden atypische Neuroleptika, teilweise auch Sedativa aus dem Bereich der Benzodiazepine eingesetzt, letztere in aller Regel allerdings zeitlich begrenzt.

Über die therapeutischen Angebote und die Strukturierung der Tage gibt der Wochenplan (s. Abbildung 2) Auskunft. Die Therapieeinheiten, die für die Studienteilnehmer obligatorisch waren, sind grau unterlegt dargestellt. Fakultativ waren die Teilnahme an Skills- und Achtsamkeits- und Entspannungsgruppe sowie dem Training sozialer Kompetenzen.

\footnotetext{
${ }^{3}$ Die Oberärztin ist die Verfasserin der vorliegenden Arbeit, die Supervision wurde von Herrn Prof. Streeck in Göttingen durchgeführt.
} 


\begin{tabular}{|c|c|c|c|c|c|c|c|c|c|c|c|c|c|}
\hline 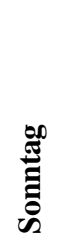 & 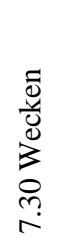 & & 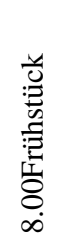 & \multicolumn{2}{|l|}{ 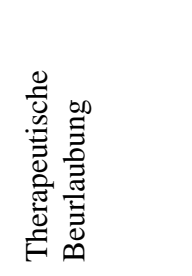 } & & & 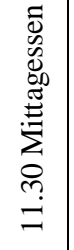 & 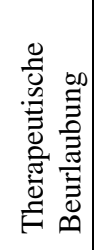 & & & & 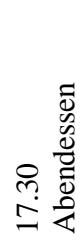 \\
\hline 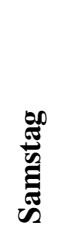 & 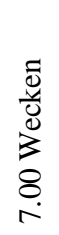 & & 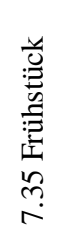 & \multicolumn{2}{|l|}{ 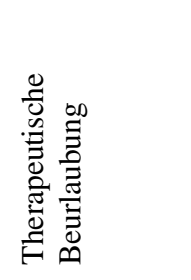 } & & & 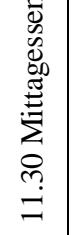 & 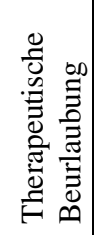 & & & & \\
\hline 离 & 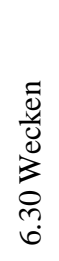 & 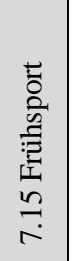 & 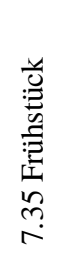 & 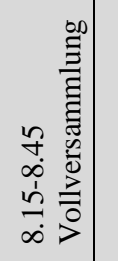 & 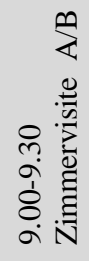 & 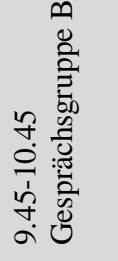 & 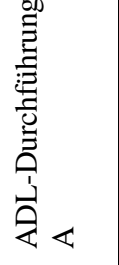 & 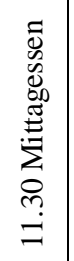 & 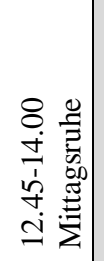 & 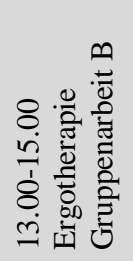 & \multicolumn{2}{|c|}{ 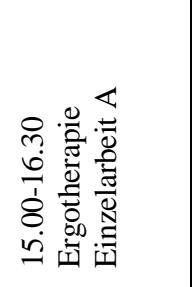 } & r. \\
\hline 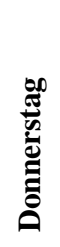 & 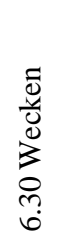 & 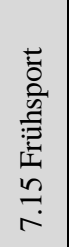 & 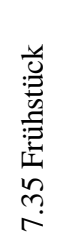 & \multicolumn{2}{|c|}{ 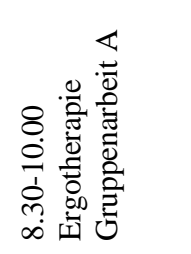 } & 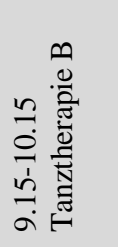 & 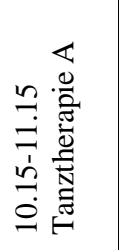 & 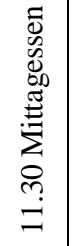 & 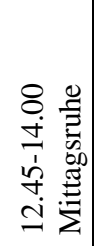 & 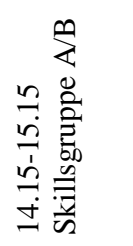 & \multicolumn{2}{|l|}{ 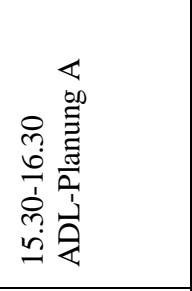 } & 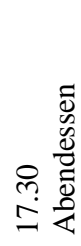 \\
\hline 离 & 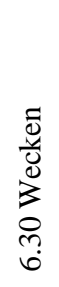 & 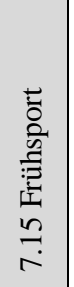 & 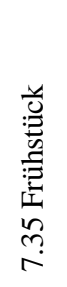 & \multicolumn{2}{|l|}{ 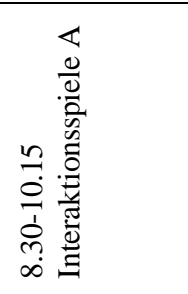 } & 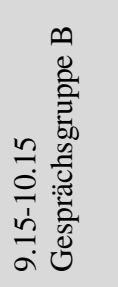 & 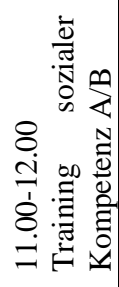 & 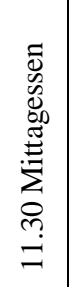 & 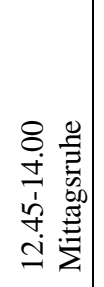 & 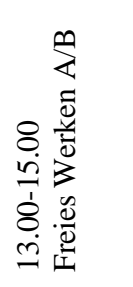 & & & \\
\hline 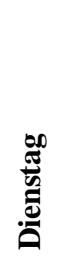 & 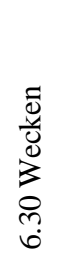 & 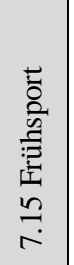 & 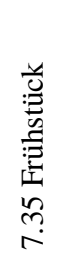 & \multicolumn{2}{|c|}{ 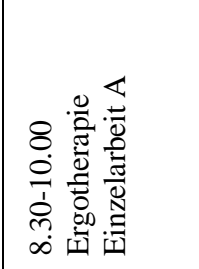 } & 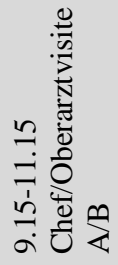 & 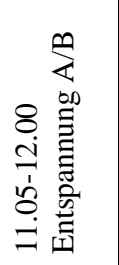 & 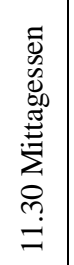 & 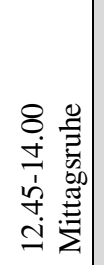 & 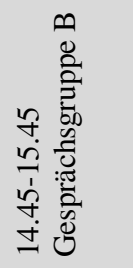 & \multicolumn{2}{|l|}{ 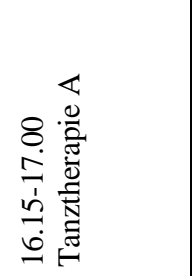 } & 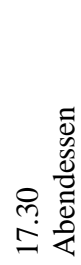 \\
\hline & 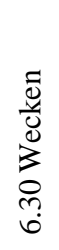 & 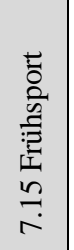 & 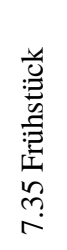 & 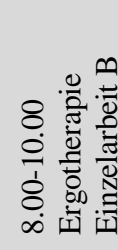 & & 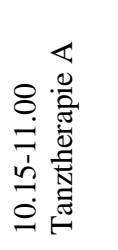 & & 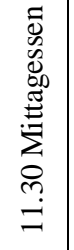 & 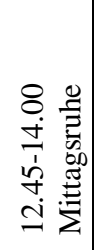 & 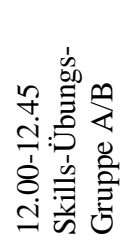 & 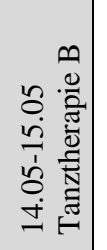 & 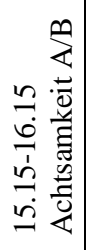 & 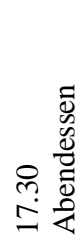 \\
\hline
\end{tabular}

Abb. 2: Wochenplan Klinik Hagen; A= supportive Gruppe, B=Psychotherapiegruppe 


\subsubsection{Klinik Göttingen ${ }^{4}$}

Das Asklepios Fachklinikum Göttingen, vormals Niedersächsisches Landeskrankenhaus Göttingen, ist eine Fachklinik für Psychiatrie und Psychotherapie. Es liegt im Südwesten der Stadt Göttingen und stellt die psychiatrische Pflichtversorgung für die Städte Göttingen, Northeim, Osterode (Harz) und die südwestlichen Teile des Landkreises Goslar sicher. Dieses Gebiet umfasst eine Einwohnerzahl von rund 630000.

Die vollstationäre Behandlung findet in spezialisierten Behandlungsbereichen statt. So gibt es mehrere Stationen für die Akutaufnahme, die Gerontopsychiatrie, Suchterkrankungen, Sozialpsychiatrie und Psychotherapie. Darüber hinaus wird in einem selbständigen Behandlungsbereich auch die stationäre Behandlung forensischer Patienten durchgeführt. Das Behandlungsangebot wird ergänzt durch eine gerontopsychiatrische und eine allgemeinpsychiatrisch-psychotherapeutische Tagesklinik sowie eine Institutsambulanz. Insgesamt stehen 360 Behandlungsplätze zur Verfügung.

Der Bereich Psychotherapie umfasst drei Stationen mit unterschiedlichen Behandlungsschwerpunkten und therapeutischen Angeboten. Die Behandlung der an der Studie beteiligten Patienten wurde durchgeführt auf der Station 4.1, einer Station mit 18 Behandlungsplätzen, deren Krankheitsverständnis psychodynamisch ausgerichtet ist und deren methodisches Konzept sich orientiert an der Dialektisch-Behavioralen Therapie (DBT).

Vor der Aufnahme werden zunächst die stationäre Behandlungsindikation, ausreichende Motivationslage und kognitive Fähigkeiten in einem Vorgespräch geprüft; das diagnostische Spektrum umfasst vorwiegend psychogene Störungen und schwere Persönlichkeitsstörungen. Ausschlusskriterien für eine Aufnahme sind eine manifeste Suchterkrankung oder eine akute Psychose.

Der individuell auf die jeweiligen Erfordernisse der Patienten abgestimmte Therapieplan wird nach einer eingehenden psychotherapeutisch-psychiatrischen Diagnostik erstellt. In dieser Zeit, die die erste bis zweite Behandlungswoche umfasst, erhalten die Patienten diagnostische und therapeutische Einzelgespräche durch einen Assistenzarzt in Weiterbildung oder eine Psychologin, mit einer Dauer von insgesamt 2,5 Stunden pro Woche, im weiteren Behandlungsverlauf beträgt die Einzeltherapie eine Stunde pro Woche. Je nach Störungsbild werden diese Sitzungen auch fraktioniert durchgeführt, die Behandlung findet unter regelmäßiger oberärztlicher Supervision statt. Zur Sicherstellung fachgerechter Anwendung der Dialektisch-Behavioralen Therapie wurden durch die „Arbeitsgemeinschaft Wissenschaftliche Therapie“ Schulungen auf der Station als In-House-Schulung durchgeführt, an denen das gesamte Behandlungsteam beteiligt war.

Die therapeutischen Maßnahmen bestehen in der Vermittlung von Selbstmanagementtechniken zum Umgang mit den jeweils störungsspezifischen dysfunktionalen Copingstrategien bzw. Symptombildungen der Patienten. Diese, vorwiegend im Gruppensetting angebotene Behandlung, die jeweils im Einzelsetting überprüft und vertieft

\footnotetext{
${ }^{4}$ Die Angaben wurden verfasst unter Einbeziehung persönlicher Mitteilungen von Herrn Dr. Herbold, Oberarzt der Klinik für Psychiatrie und Psychotherapie des Asklepios Fachklinikums Göttingen
} 
wird, thematisiert unter anderem die Verbesserung der Stresstoleranz, der Selbstfürsorge, des Umgangs mit Gefühlen und der zwischenmenschlichen Beziehungen; wichtiger Bestandteil der Behandlung ist auch die Einübung kontrollierter Regression, um malignen Spontanregressionen im stationären Setting und im Alltagsleben entgegen zu wirken. Konfliktzentrierte Gruppentherapien gehören nicht zum Behandlungsangebot.

Bei entsprechender Indikation können Paar- oder Familiengespräche, sozialarbeiterische Unterstützung sowie Psychopharmakotherapie das Behandlungsangebot ergänzen. Die Behandlung ist multiprofessionell; an ihr beteiligt sind neben ärztlichen und psychologischen Psychotherapeuten Mitarbeiter aus Krankenpflege, Sozialarbeit, Ergotherapie und Körpertherapie. In regelmäßigen Teamsitzungen stimmen die einzelnen Therapeuten ihr Handeln aufeinander ab.

Einen Überblick über die therapeutischen Angebote auf der Station bietet der Wochenplan der Station, der aus Abbildung 3 zu entnehmen ist. 


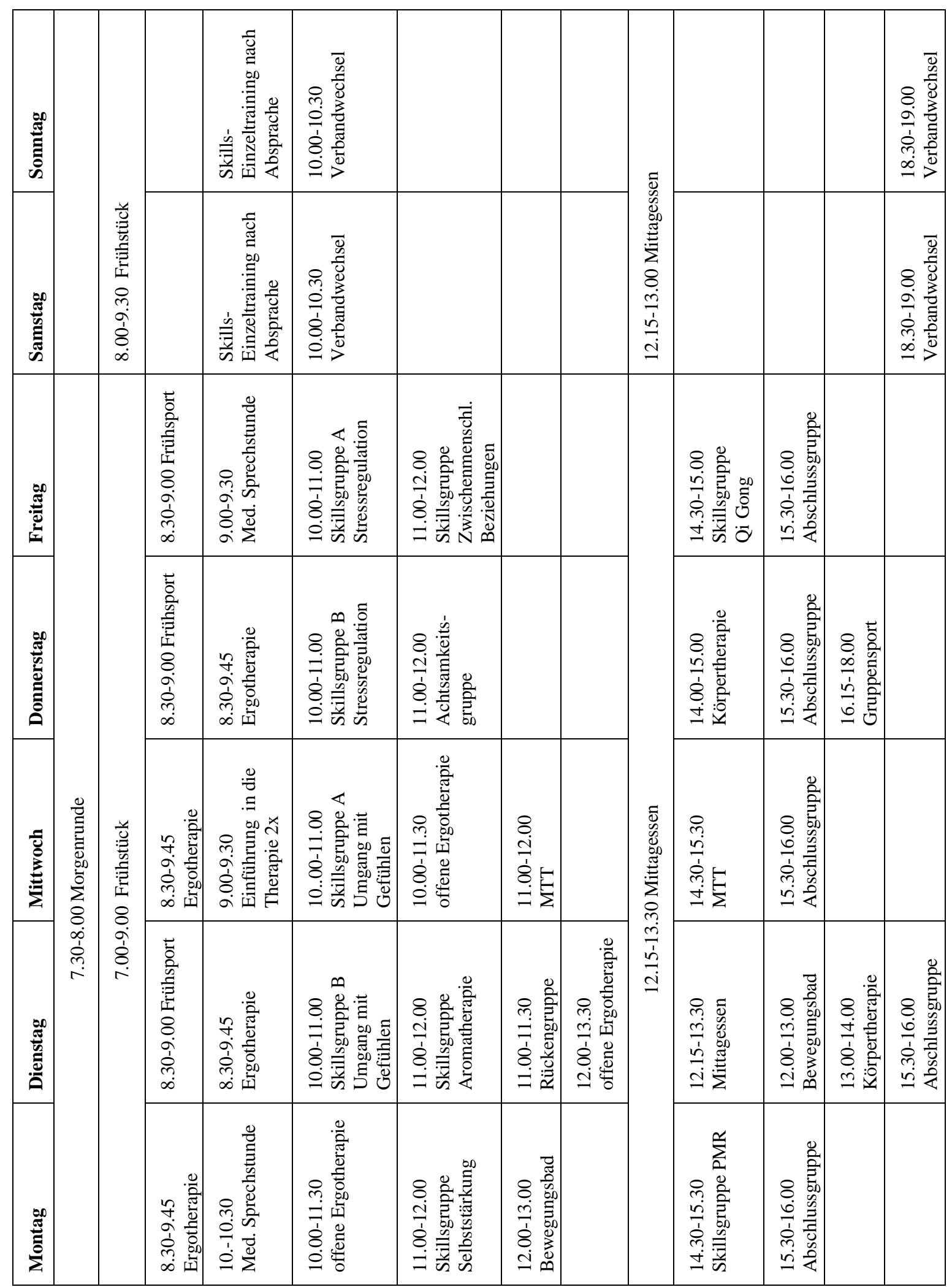

Abb. 3: Wochenplan Klinik Göttingen 


\subsection{Testinstrumente}

Zur Evaluation des Behandlungsergebnisses kamen vier Selbstbeurteilungsfragebögen und ein Fremdrater-Instrument zur Anwendung. Zu Beginn und zum Ende der Behandlung wurden durch die Patienten die Symptomausprägung (Symptom-Checkliste SCL90-R, Franke 2002), die interpersonellen Probleme (Inventar zur Erfassung interpersonaler Probleme, Horowitz et al. 2000) und die Lebenszufriedenheit (Fragebogen zur Lebenszufriedenheit, Fahrenberg et al. 1986) beurteilt. Durch die Therapeuten wurde die allgemeine Beeinträchtigungsschwere (Beeinträchtigungs-Schwere-Score, Schepank 1995) evaluiert. Als Verlaufsparameter und um das stationäre Behandlungsklima in die Untersuchung einzubeziehen, bewerteten die Patienten in zweiwöchigen Intervallen ihre Erfahrungen auf der Station (StationsErfahrungsbogen, Sammet und Schauenburg 1999). Die einzelnen Instrumente werden im Folgenden beschrieben.

\subsubsection{Symptom-Checkliste (SCL-90-R)}

Die Symptom-Checkliste SCL-90-R in der aktuellen Version wurde von Franke (2002) aus der Vorlage von Derogatis (1977) für den deutschsprachigen Raum entwickelt und ist ein international verbreitetes Instrument zur Messung subjektiver Beeinträchtigung durch körperliche und psychische Symptome. Sie entstand in der Absicht, Patienten über sich selbst in standardisierter und zeitökonomischer Form bezüglich ihrer psychischen Situation Auskunft geben zu lassen, mit der Möglichkeit zu wiederholten Messungen, z. B. im Rahmen einer psychologischen Diagnostik und Prä-/Postevaluation. Im deutschsprachigen Raum gibt es mittlerweile eine Vielzahl von Einsatzbereichen sowohl im Psychotherapiebereich als auch im allgemeinmedizinischen Behandlungskontext. Die Durchführung wird nach einer ausführlichen Instruktion durch den Testleiter vom Patienten selbst ohne Zeitlimit vorgenommen.

Das Instrument umfasst 90 Fragen zu Symptomen, die in ihrer Ausprägung auf einer 5stufigen Skala von $0=$ "überhaupt nicht" bis $4=$ "sehr stark" eingeordnet werden. Die Beurteilung bezieht sich dabei auf das Vorliegen entsprechender Symptome über einen Zeitraum von sieben Tagen vor der Beantwortung bis zum aktuellen Zeitpunkt.

Aus den 90 Items lassen sich neun Skalen zu unterschiedlichen Syndromen sowie drei globale Kennwerte errechnen. Die Skalen bezeichnen die Bereiche „Somatisierung“, "Zwanghaftigkeit“, „,Unsicherheit im Sozialkontakt“, ,,Depressivität“, „Ängstlichkeit“, „,Aggressivität/Feindseligkeit“, „Phobische Angst“, „Paranoides Denken“ und „Psychotizismus“. Die drei globalen Kennwerte bezeichnen die psychische Grundbelastung (GSI, „Global Severity Index“), die durchschnittliche Belastung in Bezug auf die Items, bei denen eine Belastung vorliegt, auch Antwortintensität genannt, (PSDI, „Positive Symptom Distress Index“) und die Anzahl aller Items, bei denen eine psychische Belastung festgestellt wird (PST, „Positive Symptom Total').

Für die vorliegende Untersuchung wurde zum Vergleich der beiden Patientenstichproben der GSI als Maß für die generelle psychische Belastung herangezogen. Der GSI berechnet sich 
aus der Summe der Summenwerte für die Einzelitems S1-S9, geteilt durch die Anzahl der beantworteten Items. Des Weiteren wurden Vergleiche, differenziert nach den neun Symptomskalen, durchgeführt.

\subsubsection{Inventar zur Erfassung interpersonaler Probleme (IIP)}

Das Inventar zur Erfassung interpersonaler Probleme in der aktuellen deutschsprachigen Version (Horowitz et al. 2000) wurde aus dem Inventory of Interpersonal Problems (Horowitz et al. 1988) entwickelt. Es basiert auf dem von Leary (1957) entwickelten Circumplex-Modell interpersonalen Verhaltens, das auf die Theorie von Sullivan (1953) zurückgeht. Diese Theorie besagt unter anderem, dass zwei Personen, die miteinander interagieren, ihr Verhalten gegenseitig beeinflussen. Leary (1957) nahm dies zum Anlass, ein zweidimensionales circumplexes Modell zur Klassifikation interpersonalen Verhaltens zu entwickeln. Ausgehend von diesem Modell wurden acht Skalen entwickelt, die sich kreisförmig um die orthogonalen Dimensionen Dominanz vs. Submissivität und Fürsorglichkeit vs. Feindseligkeit anordnen lassen. Die Skalenbezeichnungen lauten im Einzelnen (s. auch Horowitz et al. 2000, S. 1622):

„PA“: „zu autokratisch/dominant“

„BC“: „Zu streitsüchtig/konkurrierend“

„DE“: ,Zu abweisend/kalt““

„FG“: ,zu introvertiert/sozial vermeidend“

„HI“: „Zu selbstunsicher/unterwürfig““

„JK“: ,zu ausnutzbar/nachgiebig“"

„LM“: ,zu fürsorglich/freundlich“

„NO“: „Zu expressiv/aufdringlich“.

Interpersonale Probleme sind in psychotherapeutischen Behandlungen immer wieder von besonderer Bedeutung und häufig Anlass, sich in eine solche Behandlung zu begeben. Um diese Problematik auch für die Status- und Prozessdiagnostik in der klinischen Psychotherapieforschung messbar zu machen, wurde der IIP entwickelt, der sich mittlerweile als Standardinstrument etabliert hat. Er ist unabhängig von der Therapierichtung, kann somit sowohl für psychoanalytische, verhaltenstherapeutische, einzel- oder auch gruppentherapeutische Verfahren angewendet werden. Er liegt in einer Kurz- und einer Langversion vor, für die vorliegende Untersuchung wurde die Kurzform angewendet.

Für die Durchführung füllen die Patienten selbständig einen Fragebogen mit 64 Items zu interpersonalen Schwierigkeiten aus, die anhand einer Skala von ,0 $=$ nicht“" bis , $4=$ sehr“, eingeschätzt werden sollen. Für die Auswertung wurden die Rohsummenwerte verwendet. Hierzu werden die Antworten den acht Circumplexskalen zugeordnet und die Werte addiert. 
Neben der skalenbezogenen Auswertung wird auch ein Gesamtwert "IIPges" erhoben, der dem Mittelwert aller Skalenwerte entspricht.

\subsubsection{Fragebogen zur Lebenszufriedenheit (FLZ)}

Der Fragebogen zur Lebenszufriedenheit wurde von Fahrenberg et al. (1986) im Rahmen der Rehabilitationsforschung an einer Stichprobe von Herz-Kreislaufpatienten entwickelt und liegt mittlerweile in einer normierten Langversion (Fahrenberg et al. 2000) vor.

Bei dem Begriff der Lebenszufriedenheit handelt es sich um einen heterogenen Begriff, der sich auf verschiedene Aspekte des subjektiven Wohlbefindens bezieht. Dabei gibt es zahlreiche Untersuchungen zur Korrelation von Lebenszufriedenheit mit soziodemografischen und psychopathologischen Faktoren, die von Fahrenberg et al. (2000) in einer Übersicht dargestellt werden. Geringen Einfluss auf die Lebenszufriedenheit hat demnach das Geschlecht, wohingegen sich eine Tendenz zu höherer allgemeiner Lebenszufriedenheit mit zunehmendem Alter feststellen lässt. Positiven Einfluss haben die Faktoren Partnerschaft und soziale Unterstützung, hoher sozioökonomischer Status und Religiosität. Der Gesundheitszustand nimmt einen außerordentlich hohen Stellenwert bei der Lebenszufriedenheit ein. Auch Persönlichkeitseigenschaften korrelieren mit der allgemeinen Lebenszufriedenheit, positiv korreliert sind Extraversion und eine aktive Lebensführung, negativ korreliert Neurotizismus und Depression.

Für die vorliegende Untersuchung wurde die Kurzform des Fragebogens als Screeninginstrument für den Prä-Post-Vergleich verwendet. Grundlage ist der von Fahrenberg et al. (1986) ursprünglich entwickelte Fragebogen in leicht modifizierter Form. Da die Fragen zum Bereich „Ehe/Partnerschaft“ und „Kinder“ für viele Patienten nicht beantwortbar waren, wurden sie durch die Bereiche „Freundschaften“ und „Beziehung zu den Eltern“ ersetzt. In dieser Form wird der Fragebogen seit mehr als zehn Jahren zur Psychotherapieevaluation angewendet ${ }^{5}$.

Es wird jeweils eine Frage zu insgesamt 8 wichtigen Lebensbereichen gestellt, die in einem siebenstufigem Item ( 1 = "sehr zufrieden bis $7=$ "sehr unzufrieden") beantwortet werden soll. Die einbezogenen Lebensbereiche sind Gesundheit, Arbeit/Beruf, finanzielle Lage, Freizeit/Hobby, Freundschaften, Eltern, eigene Person und Sexualität. Zur Berechnung der allgemeinen Lebenszufriedenheit werden die Itemwerte addiert, so dass sich ein Summenwert errechnen lässt, der zwischen 7 (in allen Bereichen „sehr zufrieden“) und 56 (in allen Bereichen ,sehr unzufrieden“) liegen kann. Ein sinkender Wert im Prä-Post-Vergleich bildet somit eine subjektive Verbesserung der Lebensqualität ab.

In einem weiteren Abschnitt wird die subjektive Wichtigkeit der Bereiche ebenfalls auf einer siebenstufigen Skala abgefragt, geht aber nicht in die Auswertung mit ein.

\footnotetext{
${ }^{5}$ Im Asklepios Fachklinikum Tiefenbrunn wird diese Form des FLZ regelmäßig für Forschungszwecke verwendet.
} 


\subsubsection{Beeinträchtigungs-Schwere-Score (BSS)}

Der Beeinträchtigungs-Schwere-Score (Schepank 1995) ist ein weit verbreitetes Instrument, um die Beeinträchtigung eines Patienten durch seine psychogene Symptomatik aus Expertensicht einzuschätzen. Er ist weitgehend unabhängig von Pathogenesekonzepten und Diagnosen. Dabei weist er eine hohe Interraterreliabilität auf und gilt insbesondere für Veränderungen als spezifisch und sensibel. Er eignet sich daher sowohl für die Erfassung eines Therapieerfolgs im Sinne einer Prä-/Postevaluation als auch für Vergleichsuntersuchungen zwischen zwei Institutionen.

Die Beeinträchtigungen eines Menschen werden dabei auf drei Dimensionen bewertet, der körperlichen, der psychischen und der sozialkommunikativen. Jede Dimension wird in fünf Skalenstufen mit den Schweregraden 0-4 unterteilt, wobei „0“ keine Beeinträchtigung und „4“ eine extreme Beeinträchtigung bezeichnet. Somit lässt sich sowohl die Beeinträchtigungsschwere in den drei Dimensionen als auch in der Summe als GesamtBeeinträchtigungsschwere erfassen. Entsprechend der Größe des Summenwertes gilt nach Schepank (1995, S.10):

0-1: Optimale Gesundheit

\section{2-3: Leichtere Störung}

4-5: Deutliche Störung von Krankheitswert

6-7: Schwer beeinträchtigende Erkrankung

8-9: Außerordentlich schwere Erkrankung

10-12: In jeder Hinsicht schwerst gestört.

Um eine möglichst neutrale Einschätzung zu gewährleisten, wurde die Einschätzung vorzugsweise durch einen nicht unmittelbar mit der Behandlung befassten Einzeltherapeuten durchgeführt.

\subsubsection{Stations-Erfahrungsbogen (SEB)}

Der Stations-Erfahrungsbogen wurde von Sammet und Schauenburg (1999) als Instrument zur Erfassung relevanter Faktoren im Prozess einer stationären Psychotherapie entwickelt.

Begeben sich Patienten in stationäre psychotherapeutische Behandlung, bedeutet dies immer, dass sie mit verschiedenen therapeutischen Methoden und von einem multiprofessionellen Team behandelt werden, und dass sie Teil eines Gruppenprozesses sind, der sich zwischen den Patienten auf der Station abspielt. Der reale Lebensraum wird dabei für einen definierten Zeitraum verlassen und durch den therapeutischen Raum auf der Station ersetzt. Die Patienten müssen sich Rahmenbedingungen, die für die Behandlung erforderlich sind, anpassen und sehen sich einer Vielfalt von unterschiedlichen Beziehungsangeboten ausgesetzt. Dabei muss davon ausgegangen werden, dass die verschiedenen Beziehungserfahrungen, die Patienten auf 
der Station machen, sich wesentlich auf das Therapieergebnis auswirken, wie es bereits von Orlinsky et al. (1994) zusammenfassend dargestellt wurde. Der Fragebogen gibt eine Möglichkeit, die unterschiedlichen psychotherapeutisch relevanten Faktoren in ihrem Veränderungsprozess während der stationären Therapie einzuschätzen. Der StationsErfahrungsbogen ist schulenunabhängig und kann somit sowohl in verhaltenstherapeutischen als auch psychodynamisch ausgerichteten Kliniken angewendet werden.

Es handelt sich hierbei um einen Selbstbeurteilungsfragebogen mit 38 Items, die auf einer sechsstufigen Skala von „stimmt gar nicht“ bis „stimmt genau“ einzuschätzen sind. Die Einzelitems lassen sich sieben Skalen zuordnen, die unterschiedliche Faktoren einer stationären Therapie betreffen (s. auch Sammet und Schauenburg 1999, S.9-10):

„, Skala Selbstwirksamkeit" $(G K E)^{6}$ : Einschätzung der eigenen Kompetenz und der Möglichkeit, sich selbst und die Umwelt aus eigener Kraft zu beeinflussen

„Skala Beziehung zum therapeutischen Team" (TEA): Bezeichnet die Beziehung zum Stationsteam in seiner Gesamtheit

„Skala Beziehung zum Einzeltherapeuten“ (BEZ): Berücksichtigt den Einzeltherapeuten als zentrale Beziehungsperson mit speziellen Aufgaben der Integration und Koordination der Behandlung

„, Skala Gruppenklima“ (KLI): Die Einschätzung der Atmosphäre zwischen den Patienten

„Skala Zuwendung durch Mitpatienten/ Kohäsion“ (ZUW): Beschreibt, inwieweit sich der Patient mit den Mitpatienten verbunden und unterstützt fühlt.

„, Skala Angemessenheit der Behandlungsintensität“ (INT): Beurteilt die Angemessenheit des therapeutischen Angebots im Sinne der Therapiedosis

„Skala Akzeptanz der therapeutischen Rahmenbedingungen/Stationsordnung “ (REG): Erfassung der Anpassungsfähigkeit an das Regelwerk.

Da es um die Erfassung eines Entwicklungsprozesses geht, werden die Fragebögen in definierten Zeitabständen -, die Autoren empfehlen wöchentlich, halten aber auch größere Abstände für legitim -, von den Patienten ausgefüllt. Um Antworten im Sinne der sozialen Erwünschtheit zu minimieren, versehen die Patienten die Bögen mit einem Codewort, so dass keine Rückschlüsse auf die Person während der Behandlung möglich sind. Die Zuordnung von Codewort zu Patientennamen findet erst nach Abschluss der Behandlung statt. In der vorliegenden Untersuchung wurden die Stations-Erfahrungsbögen bei Aufnahme und Entlassung sowie in 14-tägigen Intervallen während der stationären Behandlung von den Patienten bearbeitet.

Für die Auswertung werden die Einzelitems den entsprechenden Skalen zugeordnet und die Summenwerte der Skalen gemittelt. Um die Ergebnisse besser einordnen zu können, wurden von Sammet und Schauenburg (1999) Mittelwert und Streuung aus einer Stichprobe von 242 Patienten aus elf psychotherapeutischen Kliniken berechnet.

\footnotetext{
${ }^{6}$ GKE $=$ Generalisierte Kompetenzerwartung
} 


\subsection{Statistische Verfahren}

Die Daten wurden mit SPSS (Version 18.0) analysiert ${ }^{7}$.

Die soziodemografischen und klinischen Daten der beiden Behandlungsgruppen zum Aufnahmezeitpunkt wurden für dichotome Merkmale mittels $\chi^{2}$-Tests und für kontinuierliche Merkmale durch t-Tests verglichen.

Zur Ermittlung der Prä-Post-Effekte in den Selbst-und Fremdbewertungsinstrumenten (SCL, IIP, FLZ, BSS) wurden getrennt für die beiden Behandlungsbedingungen (Klinik) t-Tests für verbundene Stichproben durchgeführt.

Um die Unterschiede zwischen den Behandlungsbedingungen (Klinik), die Prä-Post-Effekte (Zeit) und die Interaktion beider Faktoren (Bedingung $x$ Zeit) in den Selbst- und Fremdbewertungsinstrumenten bestimmen zu können, wurde eine multivariate Varianzanalyse mit Messwiederholung durchgeführt. Daneben wurden die Aufnahmewerte der verwendeten Fragebögen zwischen den beiden Behandlungsgruppen mittels univariater Varianzanalyse (ANOVA) verglichen.

Um die Unterschiede nicht nur bezüglich ihrer Signifikanz zu bewerten, sondern auch die Größe der Unterschiede zu berücksichtigen, wurden die Effektstärken (d) für beide Gruppen ermittelt, indem die Differenz aus Aufnahme- und Entlassungswerten durch die Standardabweichung bei Aufnahme dividiert wurde (Cohen 1988).

\footnotetext{
${ }^{7}$ Die statistischen Berechnungen wurden mit freundlicher Unterstützung durch die Abteilung Dokumentation/Supervision/Fortbildung des Asklepios Fachklinikums Tiefenbrunn unter der Leitung von Herrn Dipl.Psych Jaeger und Herrn Dipl.Psych. Masuhr durchgeführt
} 


\section{Ergebnisse}

\subsection{Patientenstichprobe}

In der Klinik Hagen gingen 42, in der Klinik Göttingen 40 Patienten in die Auswertung der Studie ein. Patienten, die die Behandlung vorzeitig beendeten und/oder von denen keine Abschlussergebnisse vorlagen, wurden nicht einbezogen. Es wurde nicht erfasst, wie hoch der Anteil der Patienten war, die eine Teilnahme an der Studie ablehnten, oder aus anderen Gründen nicht an der Datenerhebung teilnahmen, ebenso ist der Anteil der Studienabbrecher nicht bekannt.

Wie bereits im vorangegangenen Kapitel erwähnt, war der Zeitpunkt der Aufnahme der Patienten in die Studie in beiden Kliniken - abhängig von den jeweiligen Behandlungsstrukturen - (geringfügig) unterschiedlich. Im Unterschied zur Göttinger Gruppe, die bereits zum Aufnahmezeitpunkt mit den Testerhebungen begann, wurden die Patienten in Hagen erst nach der Klärung der gruppentherapeutischen Behandlungsindikation um vollständige Testbearbeitung gebeten, dies betraf den IIP, den FLZ und den SEB. Die SCL gehörte demgegenüber zur allgemeinen Diagnostik und wurde von allen Patienten zeitnah zur Aufnahme beantwortet, dies galt gleichermaßen für den BSS von Therapeutenseite.

Die Erhebung soziodemografischer Daten blieb im Vergleich zwischen den Kliniken auf die Angaben zu Schulbildung, Geschlecht und Alter beschränkt. Darüber hinausgehende Informationen zu Familienstand, Berufstätigkeit oder therapeutischen Vorbehandlungen lagen nicht bei allen Patienten vor bzw. konnten im Nachhinein nicht ausreichend rekonstruiert werden, so dass diese nicht in die vergleichende Auswertung einbezogen werden konnten. Gleiches galt für die psychopharmakologische Behandlung.

\subsection{Soziodemografische Daten}

\subsubsection{Klinik Hagen}

In der Hagener Stichprobe waren 15 Patienten männlich (36\%) und 27 weiblich (64\%).

Der Altersdurchschnitt lag bei 36,05 Jahren (SD 10,21), mit einem Range zwischen 18 und 66 Jahren. 40 Patienten waren dabei zwischen 18 und 49 Jahre alt, lediglich 2 Patienten waren älter als 50 Jahre.

Bezüglich der Schulbildung überwogen Haupt- und Realschulabschlüsse (N=29; 69\%) in deutlicher Weise die Abschlüsse mit Abitur $(\mathrm{N}=13 ; 31 \%)$. Die Abbildungen 4 bis 6 veranschaulichen diese Ergebnisse. 


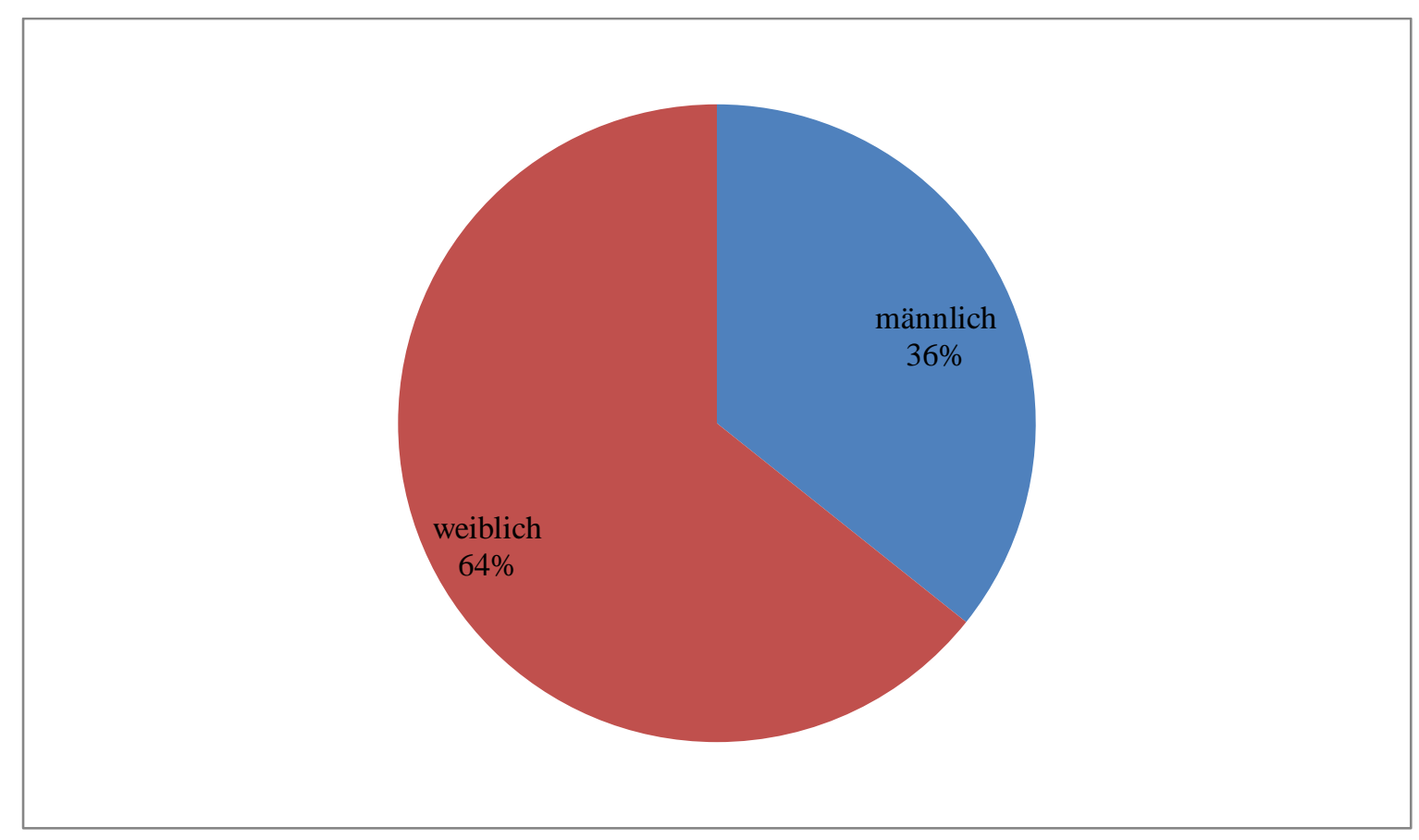

Abb. 4: Geschlechterverteilung Hagen ( $=42)$

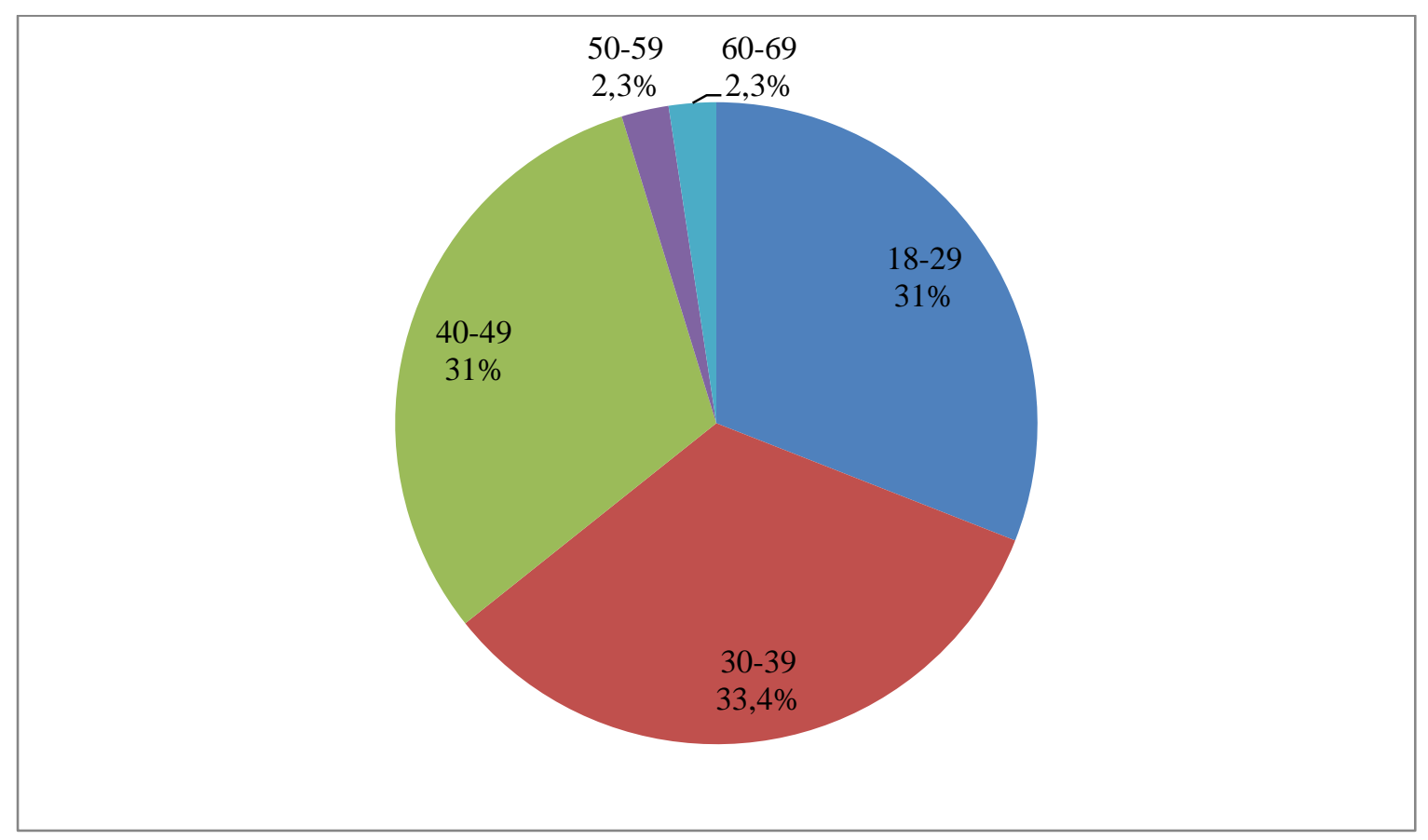

Abb. 5: Altersverteilung Hagen (Alter in Jahren, $\mathrm{N}=42$ ) 


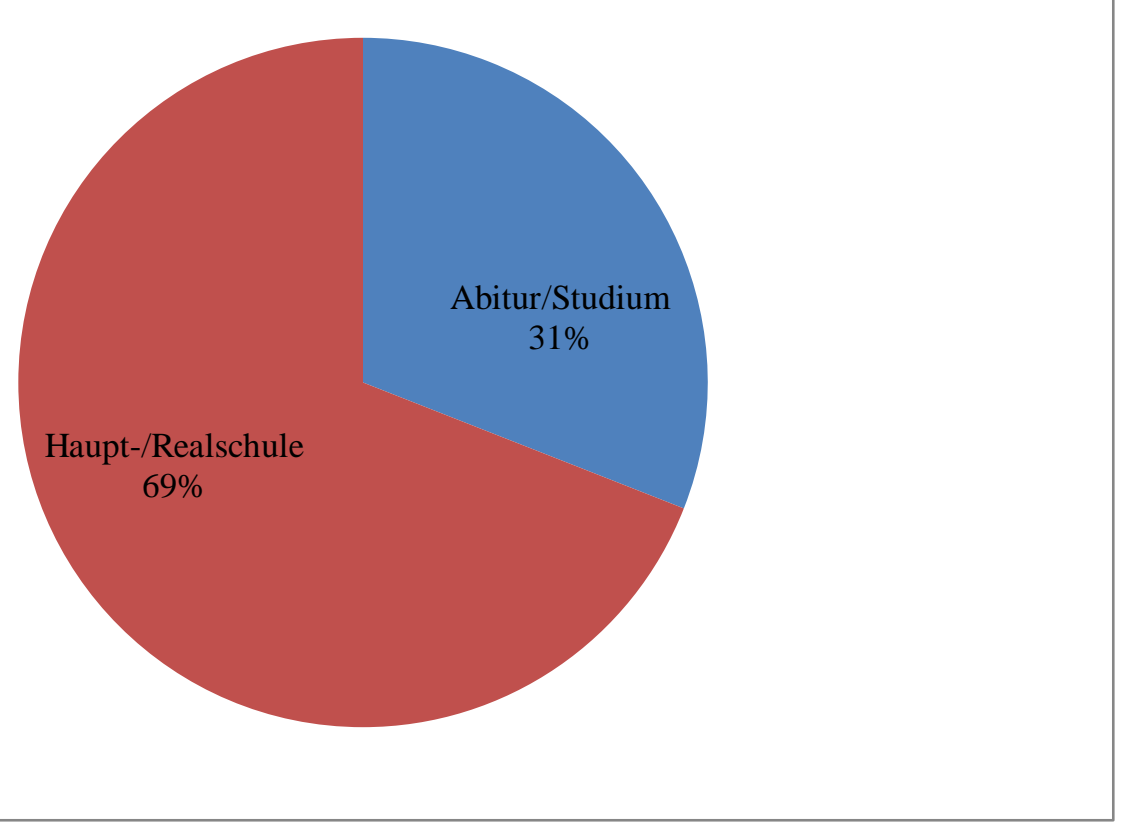

Abb. 6: Schulbildung Hagen (N=42)

\subsubsection{Klinik Göttingen}

Die Göttinger Gruppe bestand aus 28 (70\%) weiblichen und 12 (30\%) männlichen Patienten. Der Altersdurchschnitt lag bei 31,38 Jahren (SD 7,85), der Altersrange bewegte sich zwischen 19 und 49 Jahren, ein deutlicher Schwerpunkt $(\mathrm{N}=20 ; 50 \%)$ lag im Altersbereich zwischen 18 und 29 Jahren.

Bezüglich der Schulbildung hatten alle Patienten einen Schulabschluss, dabei waren Hauptund Realschulabschlüsse deutlich häufiger $(\mathrm{N}=24 ; 60 \%)$ als Abschlüsse mit (Fach-)Abitur oder Studium ( $\mathrm{N}=16 ; 40 \%)$. Diese Daten sind in den Abbildungen 7 bis 9 grafisch dargestellt. 


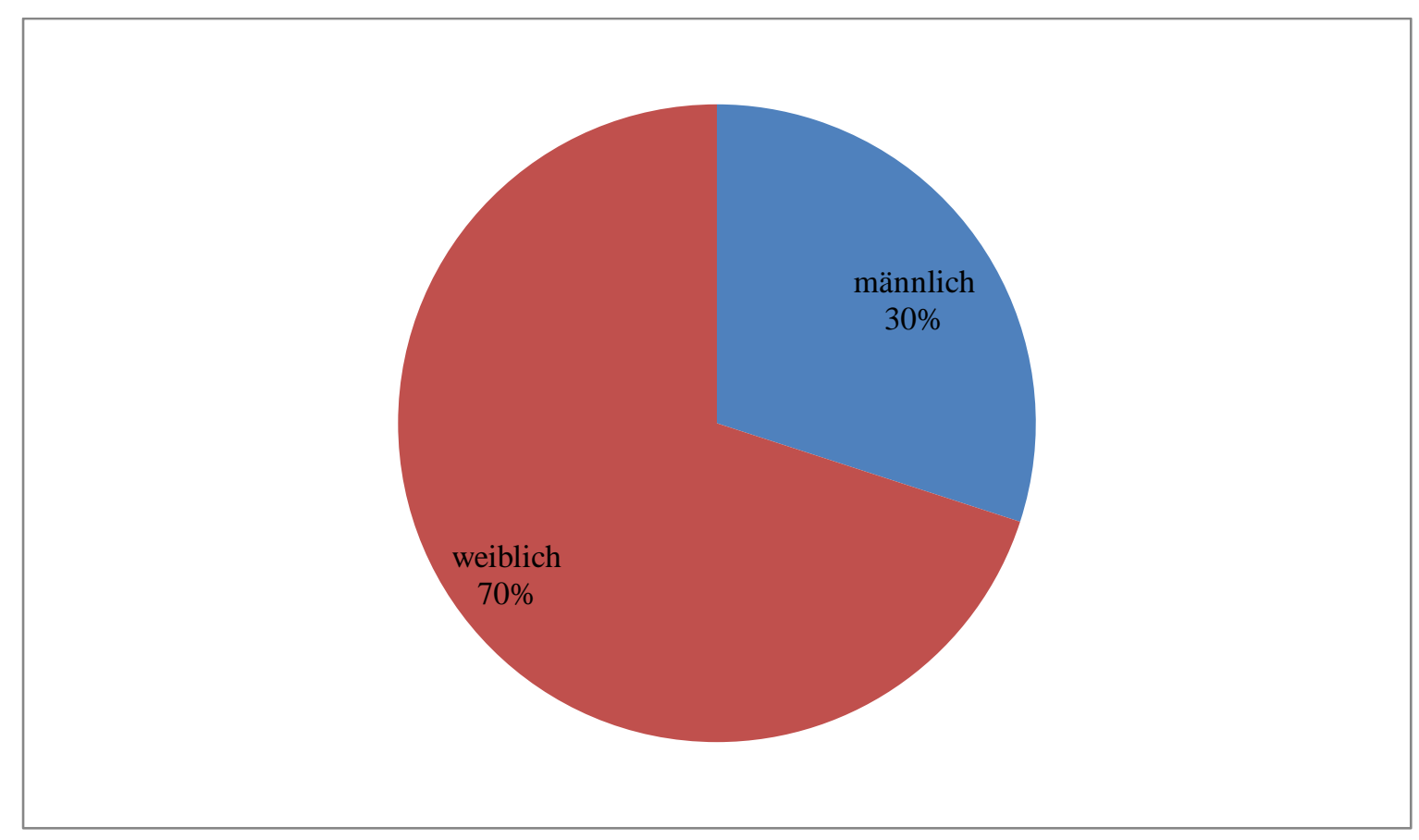

Abb. 7: Geschlechterverteilung Göttingen (N=40)

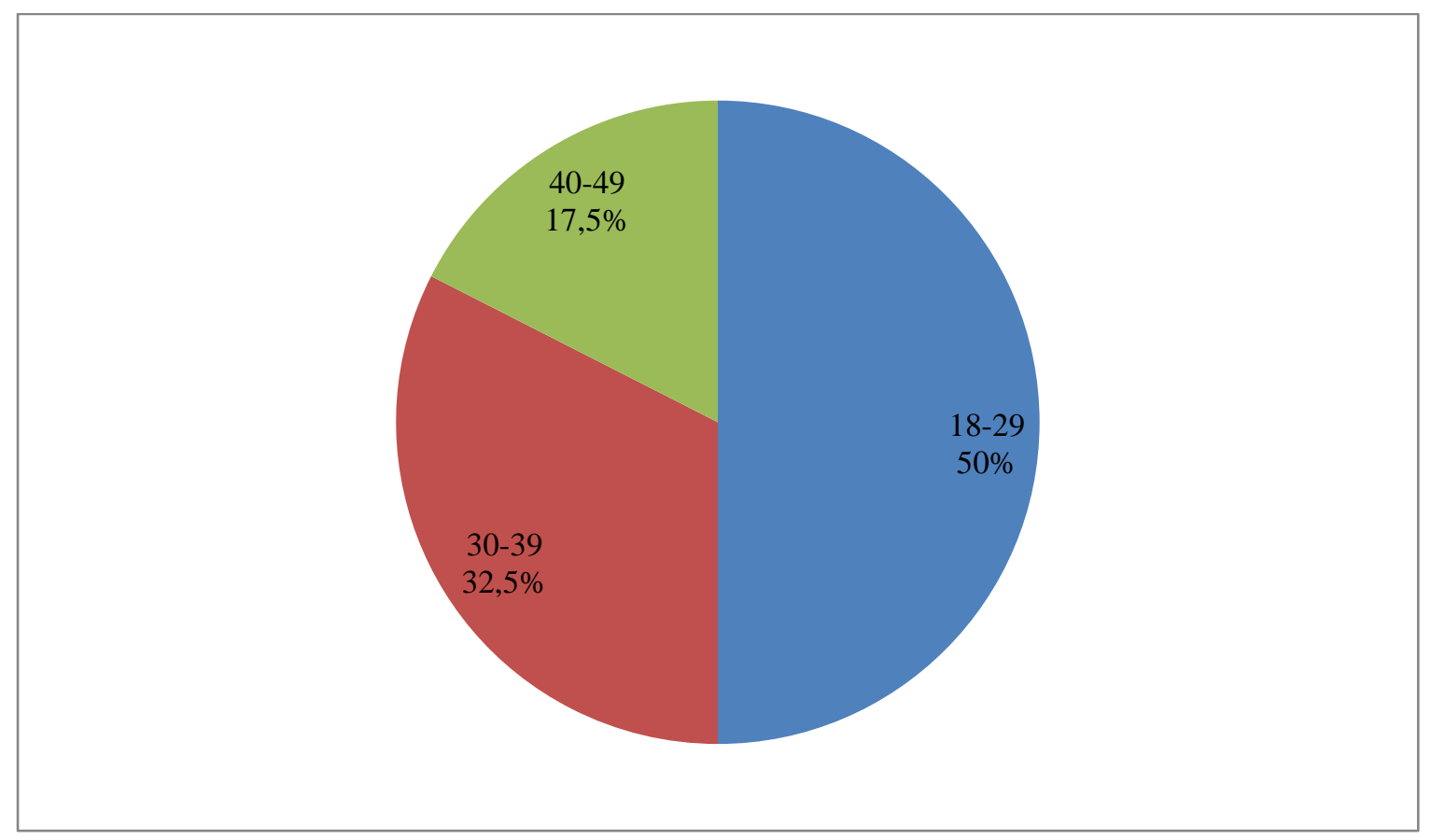

Abb. 8: Altersverteilung Göttingen (Alter in Jahren, $\mathrm{N}=40$ ) 


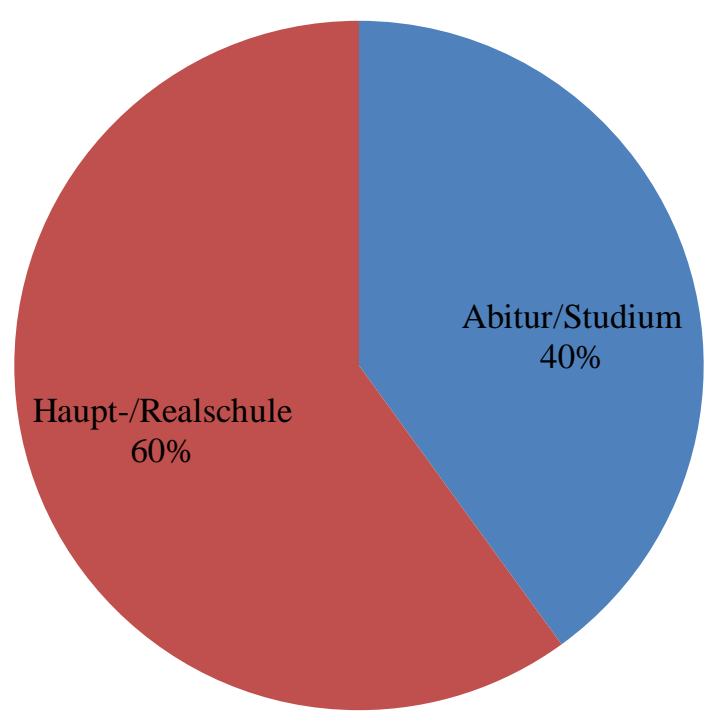

Abb. 9: Schulbildung Göttingen ( $N=40)$

\subsubsection{Vergleich der soziodemografischen Daten}

Im Geschlechterverhältnis ( $\chi^{2}$-Test: $\chi^{2}=0,303$ n.s.) gab es ebenso wie in der Schulbildung $\left(\chi^{2}=8,931\right.$ n.s.) keine signifikanten Unterschiede zwischen beiden Gruppen.

In beiden Kliniken war die Gruppe der weiblichen Patienten gegenüber der Gruppe der männlichen Patienten um ca. ein Drittel größer. Die Anzahl der Patienten mit Haupt- oder Realschulabschluss übertraf den Anteil der Patienten mit Abitur oder akademischer Ausbildung.

In der Altersverteilung gab es eine Differenz dahingehend, dass die Hagener Stichprobe mit 36,05 Jahren im Durchschnitt ein signifikant höheres Alter aufwies als die Göttinger Stichprobe mit 31,38 Jahren ( $\mathrm{T}=2,32 ; \mathrm{df}=80 ; \mathrm{p}=0,023 * 2$ 2-seitig). Der wesentliche Altersbereich lag in Hagen gleichermaßen wie in Göttingen zwischen 18 und 49 Jahren, mit einer gleichmäßigeren Verteilung der Altersstufen in Hagen. Bei den Göttinger Patienten fand sich eine Linksverschiebung in die jüngere Altersklasse. Den Vergleich der Daten zeigen die Abbildungen 10 bis 12 . 


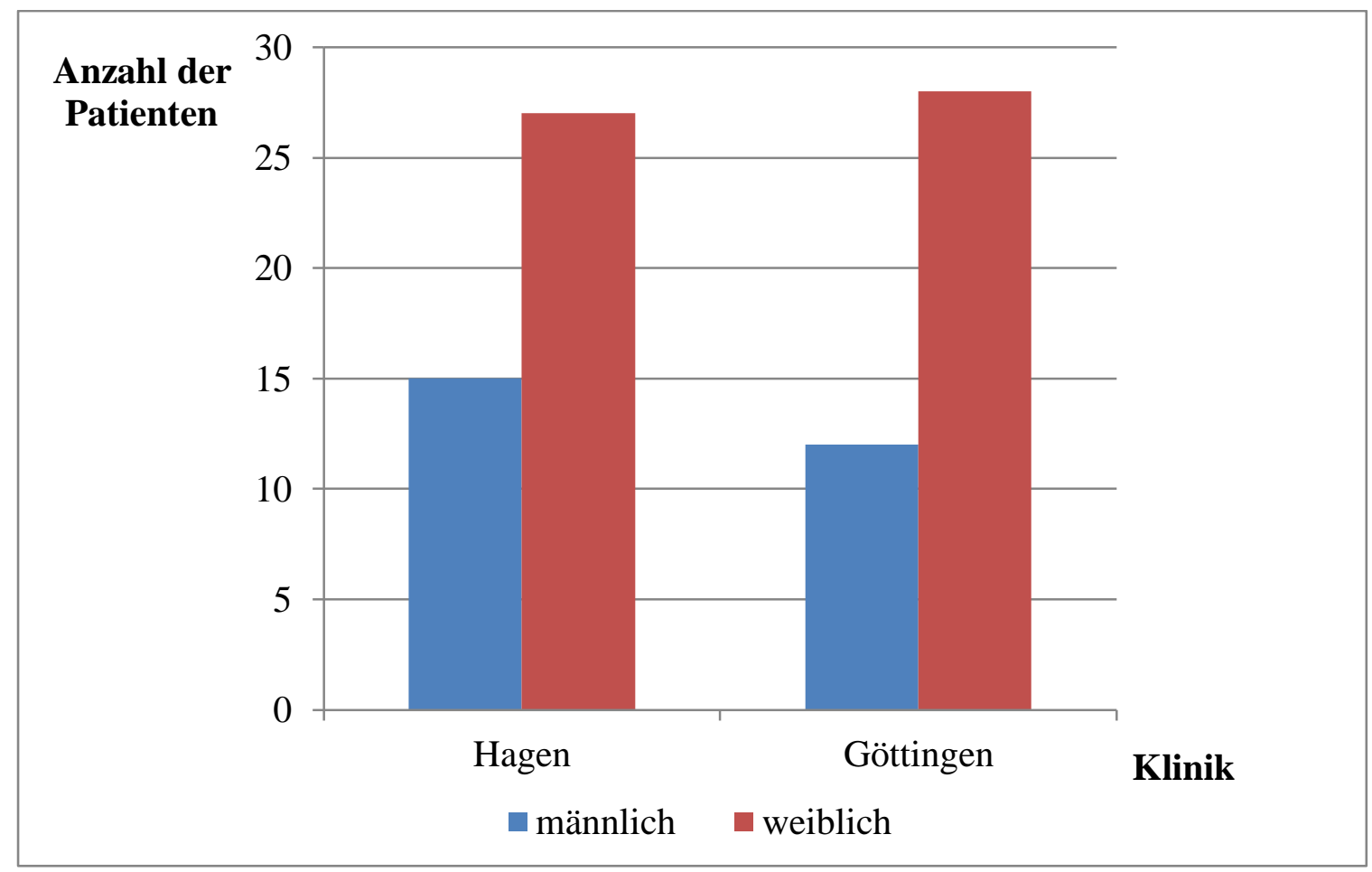

Abb. 10: Vergleich Geschlechterverteilung

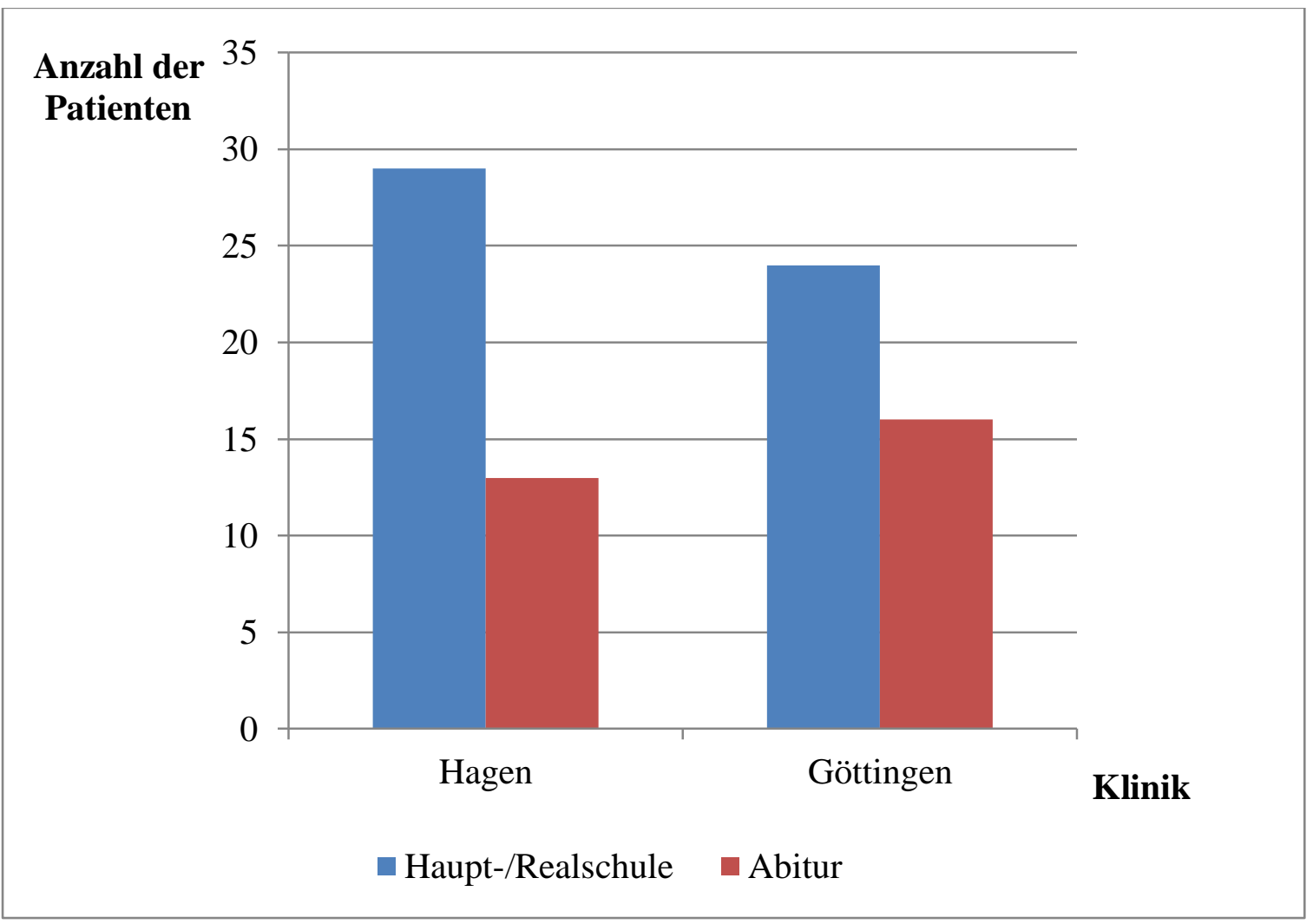

Abb. 11: Vergleich Schulbildung 


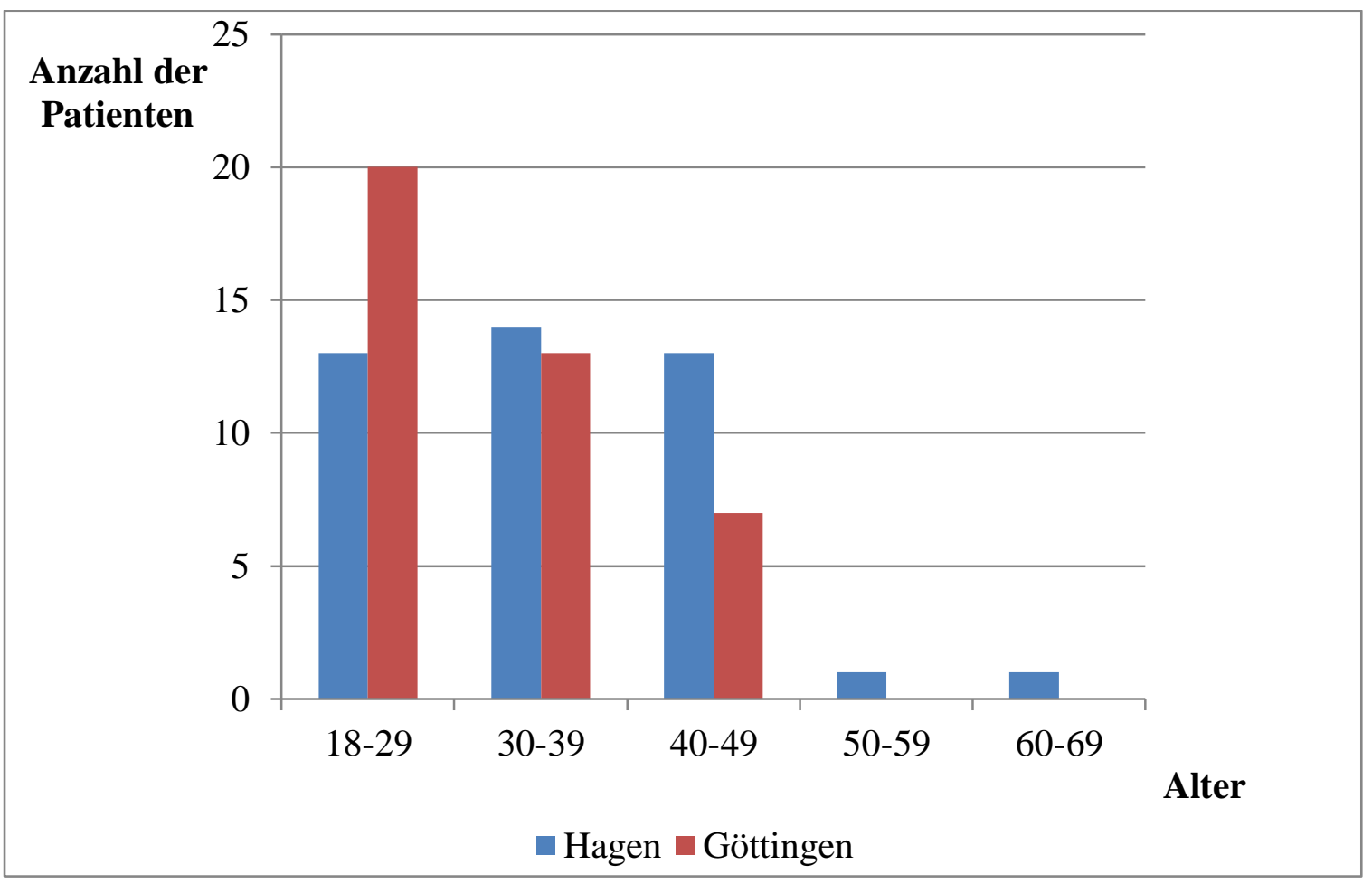

Abb. 12: Vergleich Altersverteilung

\subsection{Klinische Daten}

\subsubsection{Diagnosen}

In der Hagener Patientenpopulation wurden insgesamt 93 Diagnosen gestellt, die durchschnittliche Zahl der Komorbiditäten lag bei 2,1. Ein deutlicher Schwerpunkt lag auf den Diagnosen aus dem depressiven Bereich (F31.4, F 32.x, F33.x, F34.1, F43.2 ${ }^{8}$; N=34; $36,6 \%$ ) und aus dem Bereich der Persönlichkeitsstörungen (F60.x, F 63.0; N=33; 35,5\%). Bei den Persönlichkeitsstörungen war die Unterkategorie „emotional-instabil“ (F60.3; N=11; $11,8 \%)$ und ,andere spezifische“ (F60.8; N=10; 10,8\%) am häufigsten, gefolgt von der „ängstlich-vermeidenden“ (F60.6; N=7; 7,5\%) und ,abhängigen“ (F60.7; N=2; 2,2\%)

\footnotetext{
${ }^{8}$ Bei der Diagnose F43.2 handelt es sich definitionsgemäß um eine Anpassungsstörung auf psychosoziale Belastungen mit Symptomen ,wie sie bei affektiven Störungen (F3) (außer Wahngedanken und Halluzinationen), bei Störungen des Kapitels F4 (neurotische, Belastungs- und somatoforme Störungen) und bei den Störungen des Sozialverhaltens (F91) vorkommen“ (zitiert nach Dilling et al., 1996, S. 124). Erst durch die fünfte Stelle wird die Symptomatik näher differenziert. Somit haben wir bei der Diagnose F43.2 streng genommen keinen eindeutigen Hinweis auf eine depressive Symptomatik. Im klinischen Alltag wird die fünfte Stelle in nicht mit Forschungstätigkeiten befassten Abteilungen nur selten angegeben. In der Regel ist in der Klinik Hagen hiermit eine depressive Reaktion verschlüsselt worden (Anmerkung der Verfasserin aus eigener Tätigkeit). Sie wird daher hier unter den depressiven Syndromen aufgeführt.
} 
Persönlichkeitsstörung. „Schizoide“ und „histrionische“ Persönlichkeitsstörungen (F60.1, F60.4, jeweils $\mathrm{N}=1 ; 1,1 \%$ ) kamen vereinzelt vor. Schädlicher Substanzmissbrauch folgte in der Häufigkeit den oben genannten Diagnosegruppen (F10.x, F12.1, F13.1, F19.2, F55.2; $\mathrm{N}=13 ; 14 \%$ ). Weitere Diagnosen betrafen den Bereich der Angst- und Panikstörungen (F 40.0, F41.0; $\mathrm{N}=6 ; 6,5 \%$ ) und der somatoformen und dissoziativen Störungen (F44.2, F45.0, F45.4; $\mathrm{N}=5 ; 5,4 \%)$ sowie der posttraumatischen Belastungsstörung ( $\mathrm{F} 43.1 ; \mathrm{N}=2 ; 2,2 \%)$. Die vollständige Auflistung der Diagnosen zeigt Tabelle 1.

Tabelle 1: Diagnosen Hagen, nach absteigender Häufigkeit sortiert

\begin{tabular}{|c|c|c|}
\hline $\begin{array}{c}\text { Diagnosen } \\
\text { ICD } 10\end{array}$ & $\mathbf{N}$ & $\%$ \\
\hline F32.2 & 16 & 17,20 \\
\hline F60.3 & 11 & 11,83 \\
\hline F60.8 & 10 & 10,75 \\
\hline F33.2 & 8 & 8,60 \\
\hline F60.6 & 7 & 7,53 \\
\hline F10.2 & 5 & 5,38 \\
\hline F41.0 & 5 & 5,38 \\
\hline F10.1 & 3 & 3,23 \\
\hline F32.1 & 3 & 3,23 \\
\hline F33.1 & 3 & 3,23 \\
\hline F12.1 & 2 & 2,15 \\
\hline F43.1 & 2 & 2,15 \\
\hline F45.0 & 2 & 2,15 \\
\hline F45.4 & 2 & 2,15 \\
\hline F60.7 & 2 & 2,15 \\
\hline F13.1 & 1 & 1,08 \\
\hline F19.2 & 1 & 1,08 \\
\hline F31.4 & 1 & 1,08 \\
\hline F33 & 1 & 1,08 \\
\hline F34.1 & 1 & 1,08 \\
\hline F40.0 & 1 & 1,08 \\
\hline F43.2 & 1 & 1,08 \\
\hline F44.2 & 1 & 1,08 \\
\hline F55.2 & 1 & 1,08 \\
\hline F60.1 & 1 & 1,08 \\
\hline F60.4 & 1 & 1,08 \\
\hline F63.0 & 1 & 1,08 \\
\hline Gesamt & 93 & 100,00 \\
\hline
\end{tabular}


In Göttingen wurden insgesamt 85 Diagnosen gestellt, auch hier mit einer durchschnittlichen Anzahl der Komorbiditäten von 2,1. Alle Patienten wiesen eine Persönlichkeitsstörung (F60.x; N=40; 47,1\%) auf, eindeutiger Schwerpunkt lag hierbei auf dem Subtyp ,emotionalinstabil“" (F60.3; N=22; 25,9\%). Differenziert nach den Erscheinungsformen war der „,impulsive Typ“ (F60.30; N=17; 20,0\%) deutlich häufiger als der „Borderline Typ“ (F60.31; $\mathrm{N}=5 ; 5,9 \%$ ). Die übrigen Diagnosen aus dem Bereich der Persönlichkeitsstörung wurden als ,andere spezifische“ bezeichnet (F60.8; N=18; 21,2\%). Von den Achse-I-Störungen waren depressive Störungen, hier ausschließlich mit der Diagnose ,schwere rezidivierende Depression“ (F33.2), Panikstörungen (F41.0) sowie posttraumatische Belastungsstörungen (F43.1) im Diagnosespektrum zu finden, die in ihrer Verteilung aus Tabelle 2 hervorgehen.

Tabelle 2: Diagnosen Göttingen, nach absteigender Häufigkeit sortiert

\begin{tabular}{|c|c|c|}
\hline $\begin{array}{c}\text { Diagnosen } \\
\text { ICD 10 }\end{array}$ & $\mathbf{N}$ & \% \\
\hline 33.2 & 27 & 31,76 \\
\hline 60.8 & 18 & 21,18 \\
\hline 60.30 & 17 & 20,00 \\
\hline 41.0 & 10 & 11,76 \\
\hline 43.1 & 8 & 9,41 \\
\hline 60.31 & 5 & 5,88 \\
\hline Gesamt & 85 & 100 \\
\hline
\end{tabular}

Im Vergleich der beiden untersuchten Patientengruppen war die absolute Anzahl gestellter Diagnosen nahezu, die Anzahl der Komorbiditäten absolut identisch. Unterschiede zeigten sich im Diagnosespektrum beider Kliniken. Die Variationsbreite der Diagnosen war in Hagen deutlich höher als in Göttingen. Zwar überwogen in beiden Kliniken die Diagnosen aus dem depressiven Cluster und aus dem Cluster der Persönlichkeitsstörungen. In Göttingen waren im Unterschied zu Hagen allerdings alle Patienten von einer Persönlichkeitsstörung betroffen, in der Hagener Patientengruppe war dies nur bei rund 76\% der Patienten der Fall. Dieser Unterschied war signifikant $\left(\chi^{2}=10,847 ; p=0,001 * *\right)$. Ein weiterer Unterschied lag im Bereich der Suchtmittel und des Substanzmissbrauchs, der in der Göttinger Population nicht vorkam, in Hagen aber nahezu ein Drittel der Patienten betraf. Demgegenüber waren Diagnosen aus dem Bereich der Angststörungen und noch ausgeprägter aus dem Bereich der posttraumatischen Störung und Anpassungsstörung in Hagen gegenüber der Göttinger Klinik unterrepräsentiert. Zur Veranschaulichung dient Abbildung 13. 


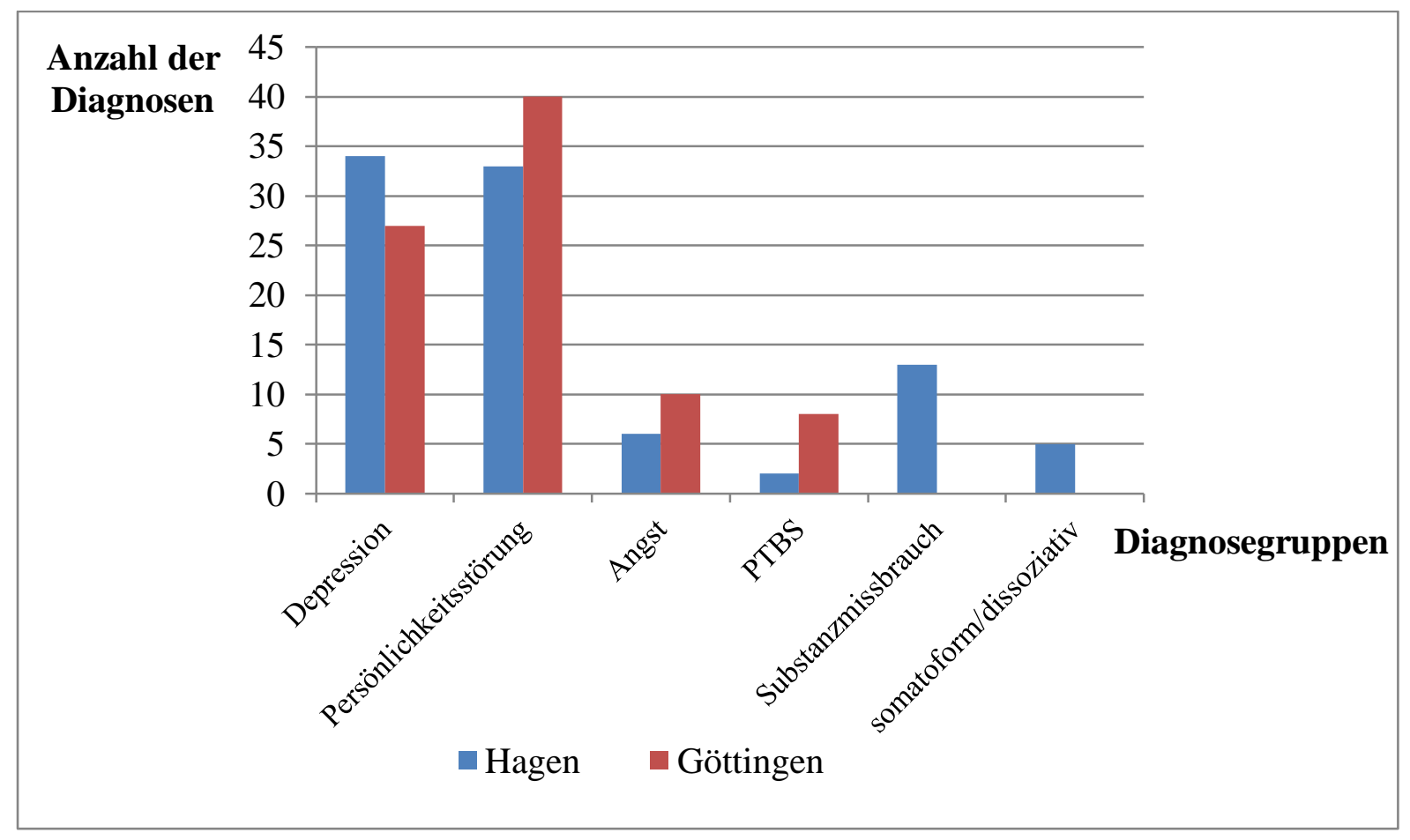

Abb. 13: Diagnoseverteilung im Vergleich

\subsubsection{Behandlungsdauer}

In Hagen betrug die durchschnittliche Behandlungsdauer durchschnittlich 7,58 Wochen (SD 1,72). Die Behandlungszeiten variierten relativ breit verteilt zwischen 4 und 11 Wochen.

In der Göttinger Patientengruppe lagen die Behandlungszeiten im Durchschnitt bei 5,45 Wochen (SD 1,20), mehr als die Hälfte der Patienten wurde in fünf Wochen behandelt.

Die Behandlungsdauer unterschied sich damit höchstsignifikant $(\mathrm{T}=-6,406 ; \mathrm{df}=78$; $\mathrm{p}<0,001^{* * *}, 2$-seitig), mit längerer Behandlungszeit in Hagen. Die Unterschiede in Dauer und Verteilung werden in Abbildung 14 verdeutlicht. 


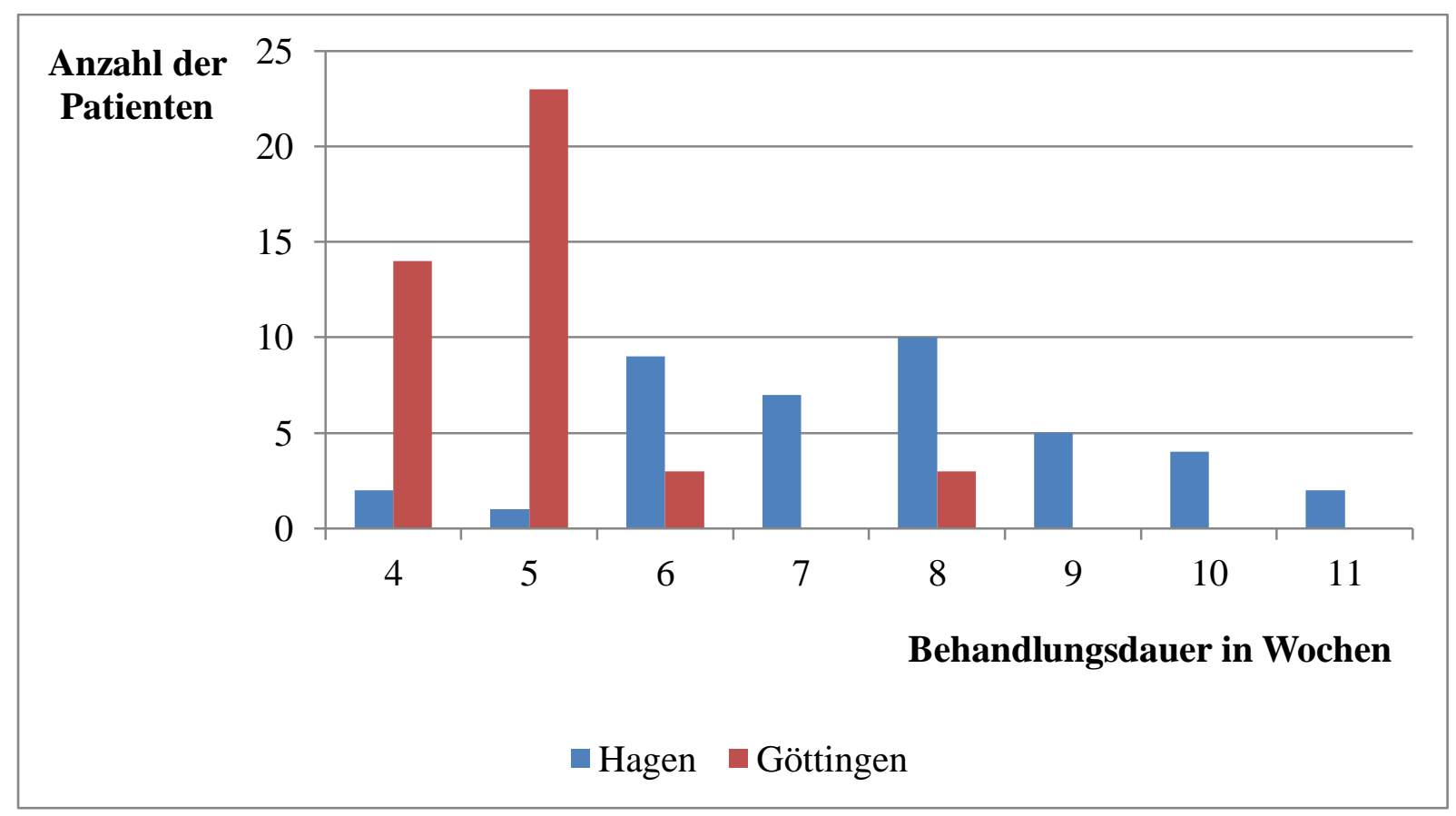

Abb. 14: Behandlungsdauer

\subsection{Testergebnisse}

Die Ergebnisse der Prä-Post-Vergleiche (Symptombelastung, interpersonelle Probleme, Beeinträchtigungsschwere und Lebenszufriedenheit) wurden zunächst - für die beiden Gruppen getrennt - berechnet. Die Veränderungen im Behandlungsverlauf (Innersubjektdifferenzen) und die Unterschiede zwischen den Kliniken (Intersubjektdifferenzen) wurden zudem varianzanalytisch untersucht. Außerdem wurden die Effektstärken (Cohen's d, Cohen 1988) ermittelt.

Für den kontinuierlichen Stationserfahrungsbogen wurde auf eine statistische Berechnung verzichtet und eine rein deskriptive Darstellung gewählt, diese erfolgt im Anschluss an die Erläuterung der Prä-Post-Untersuchungsergebnisse.

\subsubsection{Symptombelastung}

In der Hagener Gruppe zeigte sich eine deutliche Reduktion der Symptombelastung in der SCL-90-R. Sowohl der globale Kennwert GSI (Global Severity Index) als auch sieben von neun Skalen wiesen höchstsignifikante Prä-Post-Verbesserungen im Einzelvergleich auf. Die Effektstärke des GSI lag bei 1,09 und ist damit nach Cohen (1988) ${ }^{9}$ als groß zu bezeichnen. In den Subskalen war die Reduktion der Belastung für die Skala Depressivität am deutlichsten ausgeprägt mit einer großen Effektstärke von d=1,38. Ebenfalls große Effekte ergaben sich

\footnotetext{
${ }^{9}$ Nach Cohen (1988) gilt ein Effekt von $\mathrm{d} \geq 0,8$ als groß, $\mathrm{d} \geq 0,5$ als mittel und $\mathrm{d} \geq 0,2$ als klein.
} 
ferner für die Skalen „Zwanghaftigkeit“, „Unsicherheit im Sozialkontakt“, „Ängstlichkeit“ und „Aggressivität“. Mittlere Effektstärken bestanden für „paranoides Denken“ und „Somatisierung“, kleine Effektstärken für „Psychotizismus“ und ,phobische Angst“.

In der Göttinger Gruppe ließ sich ebenfalls eine bedeutsame Reduktion der Symptombelastung nachweisen, sowohl der GSI als auch acht von neun Subskalen wiesen hoch- und höchstsignifikante Prä-Post-Mittelwertsunterschiede im Einzelvergleich auf. Der GSI hatte eine mittlere Effektstärke von $d=0,54$. In den Subskalen zeigte sich, wie in Hagen, der größte Effekt in der Skala „Depressivität“, der mit d=0,78 (mittlerer bis hoher Effekt) allerdings deutlich niedriger als dort ausfiel. Mittlere Effekte erzielte diese Gruppe ebenfalls in den Skalen „Zwanghaftigkeit“ und „Ängstlichkeit“. In den übrigen Skalen zeigten sich kleine Effekte mit Werten zwischen $\mathrm{d}=0,48$ bis $\mathrm{d}=0,25$.

Im Vergleich der beiden Stichproben zeigte sich für die Symptombelastung zum Aufnahmezeitpunkt, dass die Hagener Gruppe im GSI-Mittel als auch in sämtlichen SCLSkalen insgesamt deutlich weniger belastet in die Untersuchung startete, allerdings verfehlte dieser Unterschied im GSI knapp die Signifikanzhürde (GSI $_{\text {Ha }}$ 1,44; GSI ${ }_{\text {Göt }} 1,72$; ANOVA: $\left.\mathrm{F}=3,159 ; \mathrm{p}_{\mathrm{scl}}=0,079\right)$.

Die Behandlungseffekte waren in der Klinik Hagen in allen SCL-Skalen deutlich größer als in der Klinik Göttingen, bezogen auf den GSI war die Effektstärke sogar doppelt so groß. Der Zwischengruppeneffekt, der angibt, inwieweit sich die Prä-Post-Mittelwertsdifferenzen zwischen den beiden Stichproben unterscheiden, lag für die SCL bei d=0,44, wobei Hagen die stärkere Verbesserung (allerdings von niedrigerem Ausgangsniveau) erreichte. Dieser Unterschied war allerdings nicht signifikant, wie auch weiter unten in der multivariaten Varianzanalyse dargelegt wird (MANOVA: F=3,07; $\mathrm{p}=0,084$ n.s., siehe Tabelle 10 auf Seite $55)$.

Die Werte im Einzelnen sind in Tabelle 3 und 4 getrennt für die Kliniken dargestellt. 
Tabelle 3: Symptomcheckliste SCL-90-R Hagen

\begin{tabular}{|c|c|c|c|c|}
\hline \multicolumn{5}{|c|}{$\begin{array}{c}\text { Hagen (gepaart) } \\
\text { N=41 }\end{array}$} \\
\hline & Aufnahme & Entlassung & Prä-Post & $\begin{array}{c}\text { Prä-Post- } \\
\text { Effektgröße }\end{array}$ \\
\hline SCL Skalen & M (SD) & M (SD) & t & d \\
\hline Somatisierung & $1,15(0,71)$ & $0,63(0,75)$ & $5,46 * * *$ & 0,73 \\
\hline Zwanghaftigkeit & $1,63(0,86)$ & $0,81(0,92)$ & $5,56 * * *$ & 0,96 \\
\hline Unsicherheit & $1,66(0,96)$ & $0,80(0,90)$ & $6,03 * * *$ & 0,89 \\
\hline Depression & $2,06(0,81)$ & $0,94(0,85)$ & $7,07 * * *$ & 1,38 \\
\hline Angst & $1,39(0,80)$ & $0,71(0,88)$ & $4,79 * * *$ & 0,84 \\
\hline Aggressivität & $1,52(1,02)$ & $0,68(0,82)$ & $5,36 * * *$ & 0,82 \\
\hline Phobie & $0,93(0,81)$ & $0,61(1,07)$ & $2,57 *$ & 0,40 \\
\hline Paranoides Denken & $1,46(0,93)$ & $0,74(0,92)$ & $5,09 * * *$ & 0,77 \\
\hline Psychotizismus & $0,81(0,64)$ & $0,54(0,83)$ & $3,24 * *$ & 0,43 \\
\hline GSI & $1,44(0,64)$ & $0,73(0,74)$ & $6,50 * * *$ & 1,09 \\
\hline
\end{tabular}

${ }^{*} \mathrm{p}<0,05, * * \mathrm{p}<0,01 ; * * * \mathrm{p}<0,001$

Tabelle 4: Symptomcheckliste SCL-90-R Göttingen

\begin{tabular}{|c|c|c|c|c|}
\hline \multicolumn{5}{|c|}{$\begin{array}{c}\text { Göttingen (gepaart) } \\
\mathrm{N}=\mathbf{4 0}\end{array}$} \\
\hline & Aufnahme & Entlassung & Prä-Post & $\begin{array}{c}\text { Prä-Post- } \\
\text { Effektgröße }\end{array}$ \\
\hline SCL Skalen & M (SD) & M (SD) & $\mathbf{t}$ & d \\
\hline Somatisierung & $1,37(0,88)$ & $0,99(0,68)$ & $3,78 * *$ & 0,43 \\
\hline Zwanghaftigkeit & $1,89(0,98)$ & $1,40(0,77)$ & $4,81 * * *$ & 0,50 \\
\hline Unsicherheit & $1,91(1,04)$ & $1,42(0,81)$ & $4,38 * * *$ & 0,48 \\
\hline Depression & $2,23(0,85)$ & $1,57(0,86)$ & $6,13^{* * *}$ & 0,78 \\
\hline Angst & $1,74(0,94)$ & $1,27(0,71)$ & $4,21 * * *$ & 0,50 \\
\hline Aggressivität & $1,56(0,99)$ & $1,17(0,81)$ & $3,10 * *$ & 0,39 \\
\hline Phobie & $1,44(1,17)$ & $1,14(0,76)$ & $2,21 *$ & 0,25 \\
\hline Paranoides Denken & $1,63(0,98)$ & $1,27(0,69)$ & $3,58 * *$ & 0,37 \\
\hline Psychotizismus & $1,40(1,05)$ & $1,05(0,68)$ & $3,59 * *$ & 0,34 \\
\hline GSI & $1,72(0,81)$ & $1,28(0,68)$ & $5,43 * * *$ & 0,54 \\
\hline
\end{tabular}

${ }^{*} \mathrm{p}<0,05, * * \mathrm{p}<0,01 ; * * * \mathrm{p}<0,001$ 


\subsubsection{Interpersonelle Probleme}

Die Ausprägung interpersoneller Probleme veränderte sich in der Hagener Gruppe im Einzelvergleich höchstsignifikant mit einer mittleren Effektstärke für den Gesamtwert IIPges $(\mathrm{d}=0,68)$. Differenziert nach den dimensionalen Unterkategorien des IIP zeigten sich die deutlichsten Unterschiede in den Bereichen ,introvertiert/sozial vermeidend“ und „selbstunsicher/unterwürfig“, mit einer Effektstärke von jeweils d=0,72. Es folgten die Kategorien „fürsorglich/freundlich“ $(\mathrm{d}=0,56)$, ,ausnutzbar/nachgiebig“ $\quad(\mathrm{d}=0,51)$ und „expressiv/aufdringlich“ ( $d=0,35)$, mit abnehmender Effektstärke im Sinne einer Reduktion des Merkmals. Weniger eindeutig waren die Veränderungen in den Bereichen ,autokratisch/dominant“, „streitsüchtig/konkurrierend“ und ,abweisend/kalt“.

Bei den Göttinger Patienten zeigten sich im IIP nur geringe Veränderungen, der Gesamtwert IIPges verfehlte dabei knapp eine niedrige Effektstärke $(d=0,19)$, das Ergebnis erreichte nicht das Signifikanzniveau. In den Unterkategorien ließ sich eine signifikante Veränderung lediglich in der Dimension ,selbstunsicher/unterwürfig“ erreichen, die Effektstärke war niedrig $(\mathrm{d}=0,42)$. Die übrigen Merkmalsausprägungen veränderten sich nur gering, allenfalls die Kategorien ,introvertiert/sozial vermeidend“ und ,ausnutzbar/nachgiebig“ waren nahe an einer niedrigen Effektstärke (d=0,19). In den Kategorien „streitsüchtig/konkurrierend“ und „abweisend/kalt“" kam es zu einer geringgradigen Zunahme der Werte.

Der Vergleich zwischen den Kliniken zeigte auch im Bereich der interpersonellen Probleme, dass die Hagener Gruppe insgesamt mit geringeren Werten in die Untersuchung einstieg als die Göttinger Gruppe (IIPges ${ }_{\text {Ha }} 14,15$; IIPges $_{\text {Göt }} 15,21$; ANOVA: F=1,50; $p_{\text {iip }}=0,2259$ n.s.), allerdings war der Unterschied nicht signifikant. Diese Differenz betraf vor allem die Kategorien ,abweisend/kalt“, „expressiv/aufdringlich“, „,ausnutzbar/nachgiebig“ und „selbstunsicher/unterwürfig“, am wenigsten unterschiedlich war die Kategorie „autokratisch/dominant“. Die Veränderung des Gesamtwertes IIPges im Verlauf der Behandlung war in der Hagener Gruppe varianzanalytisch signifikant größer als in der Göttinger Gruppe (MANOVA: F=4,51, p<0,05), wie aus Tabelle 10 (Seite 55) ersichtlich. Alle Werte im Vergleich für die Subkategorien in den beiden Kliniken sind in Tabelle 5 und 6 aufgelistet. 
Tabelle 5: Inventar interpersonaler Probleme Hagen

\begin{tabular}{|c|c|c|c|c|}
\hline \multicolumn{5}{|c|}{$\begin{array}{c}\text { Hagen (gepaart) } \\
\mathrm{N}=42\end{array}$} \\
\hline & Aufnahme & Entlassung & Prä-Post & Prä-Post-Effektgröße \\
\hline IIP Kat. & M (SD) & M (SD) & $\mathbf{t}$ & d \\
\hline PA & $9,00(5,68)$ & $7,57(4,85)$ & 1,54 & 0,25 \\
\hline $\mathrm{BC}$ & $11,40(5,62)$ & $10,31(5,66)$ & 1,14 & 0,19 \\
\hline $\mathrm{DE}$ & $11,90(5,81)$ & $10,90(5,62)$ & 1,39 & 0,17 \\
\hline FG & $16,69(6,69)$ & $11,86(6,35)$ & $5,42 * * *$ & 0,72 \\
\hline $\mathrm{HI}$ & $18,21(6,69)$ & $13,40(6,08)$ & $4,76^{* * *}$ & 0,72 \\
\hline $\mathrm{JK}$ & $16,31(6,05)$ & $13,24(5,66)$ & $2,96 * *$ & 0,51 \\
\hline LM & $18,00(5,81)$ & $14,74(6,20)$ & $3,23 * *$ & 0,56 \\
\hline $\mathrm{NO}$ & $11,64(5,73)$ & $9,62(5,69)$ & $2,59 *$ & 0,35 \\
\hline IIPges & $14,15(3,97)$ & $11,46(4,15)$ & $4,15 * * *$ & 0,68 \\
\hline
\end{tabular}

Tabelle 6: Inventar interpersonaler Probleme Göttingen

\begin{tabular}{|c|c|c|c|c|}
\hline \multicolumn{5}{|c|}{$\begin{array}{c}\text { Göttingen (gepaart) } \\
\mathbf{N}=\mathbf{4 0}\end{array}$} \\
\hline & Aufnahme & Entlassung & Prä-Post & Prä-Post-Effektgröße \\
\hline IIP Kat. & M (SD) & M (SD) & $\mathbf{t}$ & $\mathbf{d}$ \\
\hline PA & $9,10(6,07)$ & $8,88(5,99)$ & 0,29 & 0,04 \\
\hline $\mathrm{BC}$ & $12,08(4,84)$ & $12,18(5,09)$ & $-0,14$ & $-0,02$ \\
\hline $\mathrm{DE}$ & $13,93(5,6)$ & $14,53(5,07)$ & $-0,98$ & $-0,11$ \\
\hline FG & $17,20(6,95)$ & $15,85(7,49)$ & 1,59 & 0,19 \\
\hline $\mathrm{HI}$ & $19,43(8,3)$ & $15,93(7,09)$ & $3,62 * * *$ & 0,42 \\
\hline $\mathrm{JK}$ & $17,93(6,07)$ & $16,78(4,31)$ & 1,58 & 0,19 \\
\hline LM & $18,38(5,25)$ & $17,98(5,67)$ & 0,46 & 0,08 \\
\hline $\mathrm{NO}$ & $13,63(6,13)$ & $13,55(6,02)$ & 0,10 & 0,01 \\
\hline IIPges & $15,21(3,9)$ & $14,46(4,01)$ & 1,46 & 0,19 \\
\hline
\end{tabular}

$* \mathrm{p}<0,05, * * \mathrm{p}<0,01 ; * * * \mathrm{p}<0,001$ 


\subsubsection{Lebenszufriedenheit}

Im Bereich der Lebenszufriedenheit kam es in der Hagener Gruppe zu einer Verbesserung im Verlauf der Behandlung. Die Effektstärke war mit d=0,6 mittelgradig ausgeprägt, das Ergebnis höchstsignifikant.

In Göttingen kam es ebenfalls zu einer Verbesserung der Lebenszufriedenheit, diese war mit einem Effekt von d=0,83 groß und erreichte ebenfalls höchstes Signifikanzniveau.

Vergleichbar mit den übrigen Selbstbeurteilungsfragebögen war zwar auch in diesem Bereich die Hagener Gruppe bei Aufnahme zufriedener als die Göttinger Gruppe (FLZ FLZ $_{\text {Göt }}$ 37,5; ANOVA: F=2,78; $p_{\text {flz }}=0,099$ ), die Differenz ist jedoch nicht signifikant. Die Verbesserung der Lebenszufriedenheit im Behandlungsverlauf war bei den Göttinger Patienten ausgeprägter als in Hagen, jedoch war auch hier der Unterschied nicht signifikant (MANOVA: F=0,58; p=0,448 n.s., siehe Tabelle 10 auf Seite 55). Die einzelnen Werte im Vergleich lassen sich Tabelle 7 entnehmen.

Tabelle 7: Lebenszufriedenheit Hagen und Göttingen

\begin{tabular}{|c|c|c|c|c|}
\hline & Aufnahme & Entlassung & Prä-Post & $\begin{array}{c}\text { Prä-Post- } \\
\text { Effektgröße }\end{array}$ \\
\hline & M (SD) & M (SD) & t & d \\
\hline FLZ Hagen N=41 & $34,51(9,24)$ & $28,95(10,47)$ & $4,29 * * *$ & 0,60 \\
\hline FLZ Göttingen N=40 & $37,5(5,29)$ & $33,13(7,22)$ & $3,88^{* * * *}$ & 0,83 \\
\hline
\end{tabular}

$\mathrm{p}<0,05, * * \mathrm{p}<0,01 ; * * * \mathrm{p}<0,001$

\subsubsection{Beeinträchtigungsschwere}

Die Verbesserung der allgemeinen Beeinträchtigungsschwere bei den Hagener Patienten im Vergleich von Aufnahme zu Entlassung war das deutlichste Ergebnis der Studie. Der Effekt war mit 3,29 sehr groß und das Ergebnis höchstsignifikant. Größten Anteil an der Gesamtbeeinträchtigungsschwere bei Aufnahme hatte die psychische Beeinträchtigung mit knapp 40\% des Gesamtwertes, diese besserte sich auch im Verlauf der Behandlung am stärksten $(d=2,47)$. Der Einfluss der weiteren Faktoren (sozial 33\%, körperlich 27\%) verteilte sich gleichmäßig, auch hier zeigten sich große Effektstärken zwischen t1 bei Aufnahme und t2 vor Entlassung $\left(\mathrm{d}_{\mathrm{soz}}=1,69, \mathrm{~d}_{\text {körp }}=1,30\right)$.

Die Schwere der Beeinträchtigung nahm bei den Göttinger Patienten ebenfalls ab, mit hochsignifikantem Unterschied, der Effekt lag mit $\mathrm{d}=0,49$ im mittleren Bereich. Der Hauptschwerpunkt war dabei die psychische Subkategorie, diese machte die Hälfte des 
Gesamtwertes bei Aufnahme aus und veränderte sich am deutlichsten (große Effektstärke, $\mathrm{d}=0,8$ ). Die Beeinträchtigung im körperlichen Bereich war demgegenüber von niedrigem Effekt $(\mathrm{d}=0,34)$, die sozialkommunikative Beeinträchtigung veränderte sich gering $(\mathrm{d}=0,15)$. Die Ergebnisse sind in den Tabellen 8 und 9 aufgeführt.

In diesem Fremdbeurteilungsverfahren verhielten sich die Aufnahmewerte im Vergleich zwischen den Kliniken umgekehrt zu den Selbstbeurteilungsfragebögen: Die Hagener Patienten wurden von den Therapeuten bei der Aufnahme als deutlich schwerer beeinträchtigt eingeschätzt als die Göttinger, der Eingangswert war in Hagen nahezu doppelt so hoch, die Differenz höchstsignifikant BSSges $_{\mathrm{Ha}}$ 7,93; BSSges Göt. 4,35; ANOVA: $\mathrm{F}=118,823$ $\left.\mathrm{p}_{\mathrm{bss}}=0,000^{* * *}\right)$. Dies war vor allem auf die höheren Beeinträchtigungswerte im sozialkommunikativen und körperlichen Bereich bei den Hagener Patienten zurückzuführen, die am deutlichsten ausgeprägt waren, allerdings unterschieden sich die Werte auch für die psychische Beeinträchtigung. Zum Entlassungszeitpunkt entsprachen sich hingegen die Gesamtwerte in der Hagener (BSSges 3,8) und der Göttinger Gruppe (BSSges 3,5) nahezu. Die Differenz der Ergebnisse im Behandlungsverlauf war höchstsignifikant zugunsten der Hagener Patientenstichprobe (MANOVA: F=72,67 p<0,001, siehe Tabelle 10 auf Seite 55).

Tabelle 8: Beeinträchtigungs-Schwere-Score Hagen

\begin{tabular}{|c|c|c|c|c|}
\hline \multicolumn{5}{|c|}{$\begin{array}{c}\text { Hagen (gepaart) } \\
\text { N=41 }\end{array}$} \\
\hline & Aufnahme & Entlassung & Prä-Post & $\begin{array}{c}\text { Prä-Post- } \\
\text { Effektgröße }\end{array}$ \\
\hline & & & & t \\
\hline BSS - körperlich & $2,12(0,95)$ & $0,88(0,78)$ & $8,01 * * *$ & 1,30 \\
\hline BSS - psychisch & $3,15(0,69)$ & $1,44(0,63)$ & $11,77^{* * *}$ & 2,47 \\
\hline BSS - sozial & $2,66(0,69)$ & $1,49(0,68)$ & $11,24 * * *$ & 1,69 \\
\hline BSS - gesamt & $7,93(1,25)$ & $3,80(1,52)$ & $15,21 * * *$ & 3,29 \\
\hline$* \mathrm{p}<0,05, * * \mathrm{p}<0,01 ; * * * \mathrm{p}<0,001$ & & & \\
\hline
\end{tabular}


Tabelle 9: Beeinträchtigungs-Schwere-Score Göttingen

\begin{tabular}{|c|c|c|c|c|}
\hline \multicolumn{5}{|c|}{$\begin{array}{c}\text { Göttingen (gepaart) } \\
\text { N=40 }\end{array}$} \\
\hline & Aufnahme & Entlassung & $\begin{array}{c}\text { Prä- } \\
\text { Post }\end{array}$ & $\begin{array}{c}\text { Prä-Post- } \\
\text { Effektgröße }\end{array}$ \\
\hline & M (SD) & M (SD) & t & d \\
\hline BSS - körperlich & $0,73(0,82)$ & $0,45(0,75)$ & $2,56^{*}$ & 0,34 \\
\hline BSS - psychisch & $2,20(0,56)$ & $1,75(0,49)$ & $4,46^{* * *}$ & 0,80 \\
\hline BSS - sozial & $1,43(0,81)$ & $1,30(0,69)$ & 0,96 & 0,15 \\
\hline BSS - gesamt & $4,35(1,72)$ & $3,50(1,41)$ & $3,37^{* *}$ & 0,49 \\
\hline
\end{tabular}

\subsubsection{Einflussgrößen auf die Prä-Post-Testergebnisse}

Varianzanalytisch ließ sich im Vergleich zwischen den Gruppen der Untersuchungszeitpunkt (Faktor „Zeit“) als Einflussvariable für beide Stichproben identifizieren, dieses Ergebnis war für alle Tests höchstsignifikant. Dies bedeutet, dass ein Testergebnis wesentlich davon beeinflusst wurde, ob die Untersuchung zum Aufnahme- oder Entlassungszeitpunkt erfolgte, unabhängig von der Zugehörigkeit zur Hagener oder Göttinger Gruppe, und dass sich während der Behandlung eine signifikante Veränderung in allen getesteten Bereichen eingestellt hat.

Ergänzt um den Faktor der Behandlungsbedingung als Einflussgröße (Faktor „Zeit x Bedingung“;) ergaben sich weiterhin signifikante Unterschiede für die abhängigen Variablen IIP und BSS zugunsten der Hagener Population. Hier war das Testergebnis somit nicht nur abhängig vom Untersuchungszeitpunkt, sondern auch von der Zugehörigkeit zur Behandlungsgruppe. Anders ausgedrückt: Der Einflussfaktor „Klinik Hagen“ korrelierte mit einem signifikant besseren Ergebnis in Bezug auf die interpersonellen Probleme und die Beeinträchtigungsschwere gegenüber dem Faktor „Klinik Göttingen“. Dass dies auch für das Ausmaß des Behandlungseffektes relevant war, zeigte sich in den Zwischengruppeneffekten, ein Maß für die Unterschiede der Prä-Post-Mittelwertsdifferenzen: Dieser Effekt war für den BSS mit $d=1,97$ beträchtlich und auch für den IIP deutlich $(d=0,52)$. Für die SCL war der Zwischengruppeneffekt in Hagen ebenfalls in relevanter Weise größer als in Göttingen $(d=0,44)$, verfehlte allerdings gering das Signifikanzniveau $(p=0,084)$. Für den FLZ war das Ergebnis umgekehrt: Hier war der Einflussfaktor „Klinik Göttingen“ mit einem besseren Ergebnis korreliert als der Faktor „Klinik Hagen“. Die Zwischengruppeneffekte wiesen hier aber nur eine geringe Differenz auf $(\mathrm{d}=0,15)$, ohne das Signifikanzniveau zu erreichen. Die einzelnen Ergebnisse zeigt Tabelle 10. 
Tabelle 10: Vergleich der Testergebnisse (Intergruppenvergleich)

\begin{tabular}{|c|c|c|c|c|c|c|c|c|}
\hline \multirow[t]{2}{*}{ Test } & & \multicolumn{2}{|c|}{$\begin{array}{c}\text { Göttingen } \\
(n=40)\end{array}$} & \multicolumn{2}{|c|}{$\begin{array}{l}\text { Hagen } \\
(n=40)\end{array}$} & \multicolumn{2}{|c|}{ MANOVA } & \multirow{2}{*}{$\begin{array}{c}\text { Zwischengrup } \\
\text { peneffekte } \\
\text { Differenz }\end{array}$} \\
\hline & & Prä & Post & Prä & Post & Zeit & Bed.xZeit & \\
\hline SCL & $\mathrm{M}$ & 1,72 & 1,28 & 1,43 & 0,75 & $66,10 * * *$ & \multirow{2}{*}{$\begin{array}{c}3,07 \text { n.s. } \\
(p=0,084)\end{array}$} & \multirow[t]{2}{*}{0,44} \\
\hline & SD & 0,81 & 0,74 & 0,65 & 0,69 & & & \\
\hline IIP & $\mathrm{M}$ & 15,23 & 14,48 & 14,06 & 11,53 & $15,30 * * *$ & \multirow[t]{2}{*}{$4,51 *$} & \multirow[t]{2}{*}{0,52} \\
\hline & SD & 3,90 & 4,01 & 3,98 & 4,19 & & & \\
\hline FLZ & $\mathrm{M}$ & 37,50 & 33,13 & 34,92 & 29,21 & $32,92 * * *$ & \multirow{2}{*}{$\begin{array}{c}0,58 \text { n.s. } \\
(p=0,448)\end{array}$} & \multirow[t]{2}{*}{0,15} \\
\hline & SD & 5,29 & 7,22 & 9,29 & 10,68 & & & \\
\hline BSS & $\mathrm{M}$ & 4,35 & 3,50 & 7,90 & 3,82 & $169,41 * * *$ & \multirow[t]{2}{*}{$72,67 * * *$} & \multirow[t]{2}{*}{1,97} \\
\hline & SD & 1,72 & 1,41 & 1,27 & 1,55 & & & \\
\hline
\end{tabular}

n.s. $=$ nicht signifikant, $* \mathrm{p}<0,05, * * \mathrm{p}<0,01 ; * * * \mathrm{p}<0,001 ; \mathrm{SCL}=$ Symptomcheckliste; IIP = Inventar interpersoneller Probleme; FLZ = Fragebogen zur Lebenszufriedenheit; BSS = Beeinträchtigungs-SchwereScore

\subsubsection{Stationserfahrungen}

Die Auswertung der Testergebnisse des SEB wies durch unterschiedliche Einstiegszeitpunkte in die Bearbeitung bei der Hagener Gruppe, die unterschiedliche Behandlungsdauer und die somit im Verlauf geringen Fallzahlen Probleme auf. Um die Erfahrungen auf der Station dennoch in die Untersuchung einfließen zu lassen, wurde ein rein deskriptives Vorgehen gewählt und auf statistische Analysen verzichtet. Als Verlaufsparameter wurden die Messzeitpunkte t1(Hagen $\mathrm{N}=42$, Göttingen $\mathrm{N}=40$ ) bis $\mathrm{t} 4$ (Hagen $\mathrm{N}=34$, Göttingen $\mathrm{N}=28$ ) einbezogen.

In der Hagener Stichprobe ließen sich für fast alle untersuchten Items Werte zwischen 4,8 und 5,2 ermitteln, mit insgesamt nur wenig Veränderung im Verlauf der Behandlung. Einzige Ausnahme bildete die „Generalisierte Kompetenzerwartung“, die die Selbstwirksamkeit erfasst: Hier waren die Werte niedriger, zeigten aber eine kontinuierliche Steigerung von 3,4 bis auf 4,0. Der Durchschnitt über alle Bereiche lag bei 4,82.

Die Göttinger Patienten beurteilten die Stationserfahrungen größtenteils mit Werten zwischen 4,1 und 5,2, auch hier gab es nur geringe Entwicklungen im Behandlungsverlauf. Niedrigere Werte ergaben sich auch hier für das Item „Generalisierte Kompetenzerwartung“, hier startete die Göttinger Gruppe mit 3,1 und steigerte sich in der Bewertung bis auf 4,0. Die durchschnittliche Bewertung für alle Bereiche lag bei 4,45.

Betrachtet man den Vergleich zwischen den Kliniken, stieg die Hagener Gruppe in den meisten Skalen mit leicht höheren Werten in die Behandlung ein, eine Differenz, die auch über den Verlauf erhalten blieb. In der Skala „Akzeptanz der therapeutischen Rahmenbedingungen" zeigten sich in beiden Kliniken nahezu identische Ergebnisse. Für das Item „Generalisierte Kompetenzerwartung“ verbesserten sich die Werte in der Göttinger 
Gruppe vergleichsweise deutlicher, da die Gruppe mit einem geringeren Wert als die Hagener begann, zum Zeitpunkt t4 aber zu einem gleichen Ergebnis aufschließen konnte. Die Hagener Gruppe zeigte darüber hinaus gegenüber der Göttinger Gruppe ein durchgehend höheres Zuwendungsbedürfnis.

Die Ergebnisse sind in den Abbildungen 15 bis 21 grafisch dargestellt. Zur besseren Einordnung wurden die Werte (25-75\% Quartile) der ursprünglichen Vergleichsstichprobe des SEB nach der Berechnung von Sammet und Schauenburg (1999) in die Grafik miteingefügt.

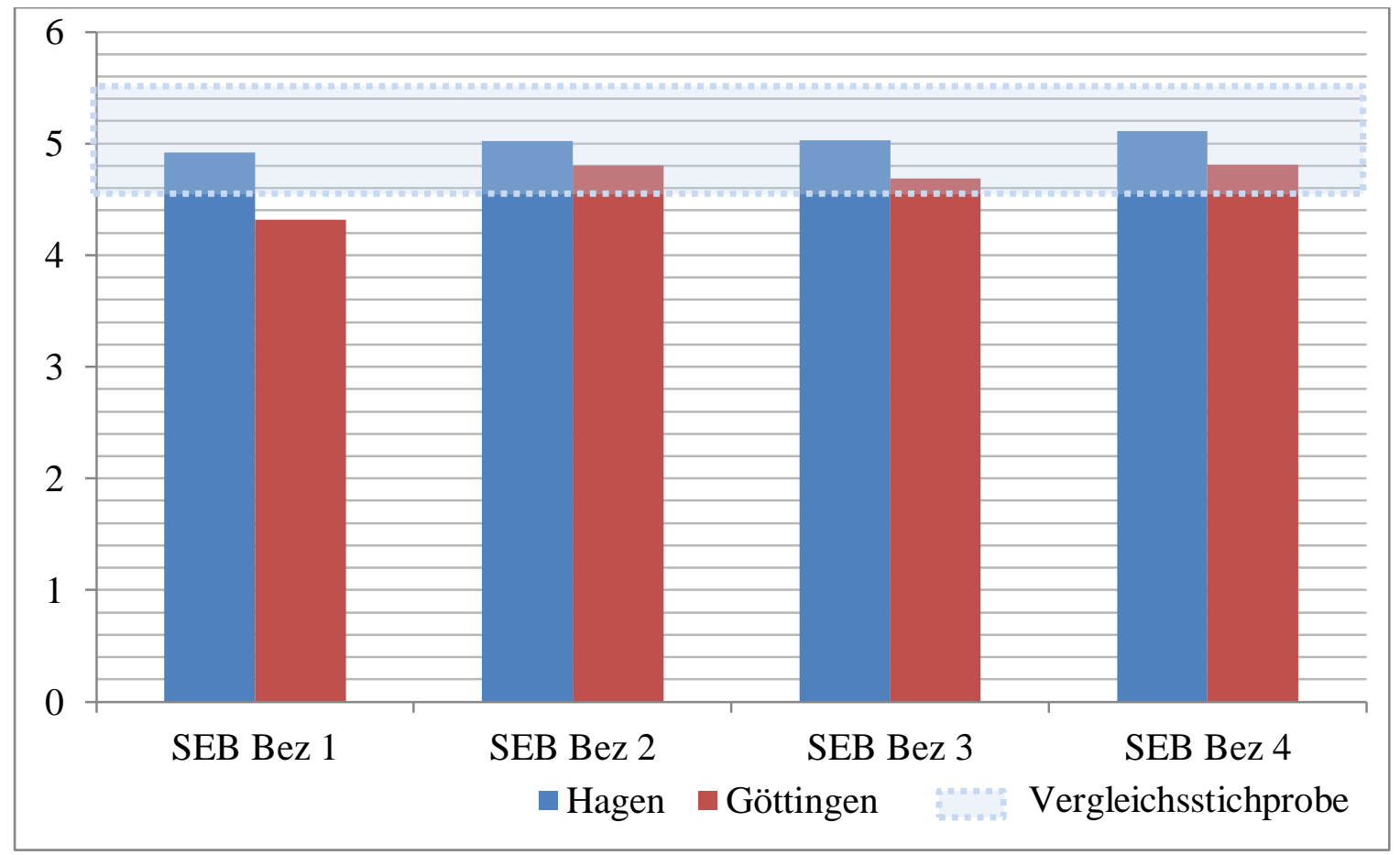

Abb. 15: Stationserfahrungsbogen: Beziehung zum Einzeltherapeuten 


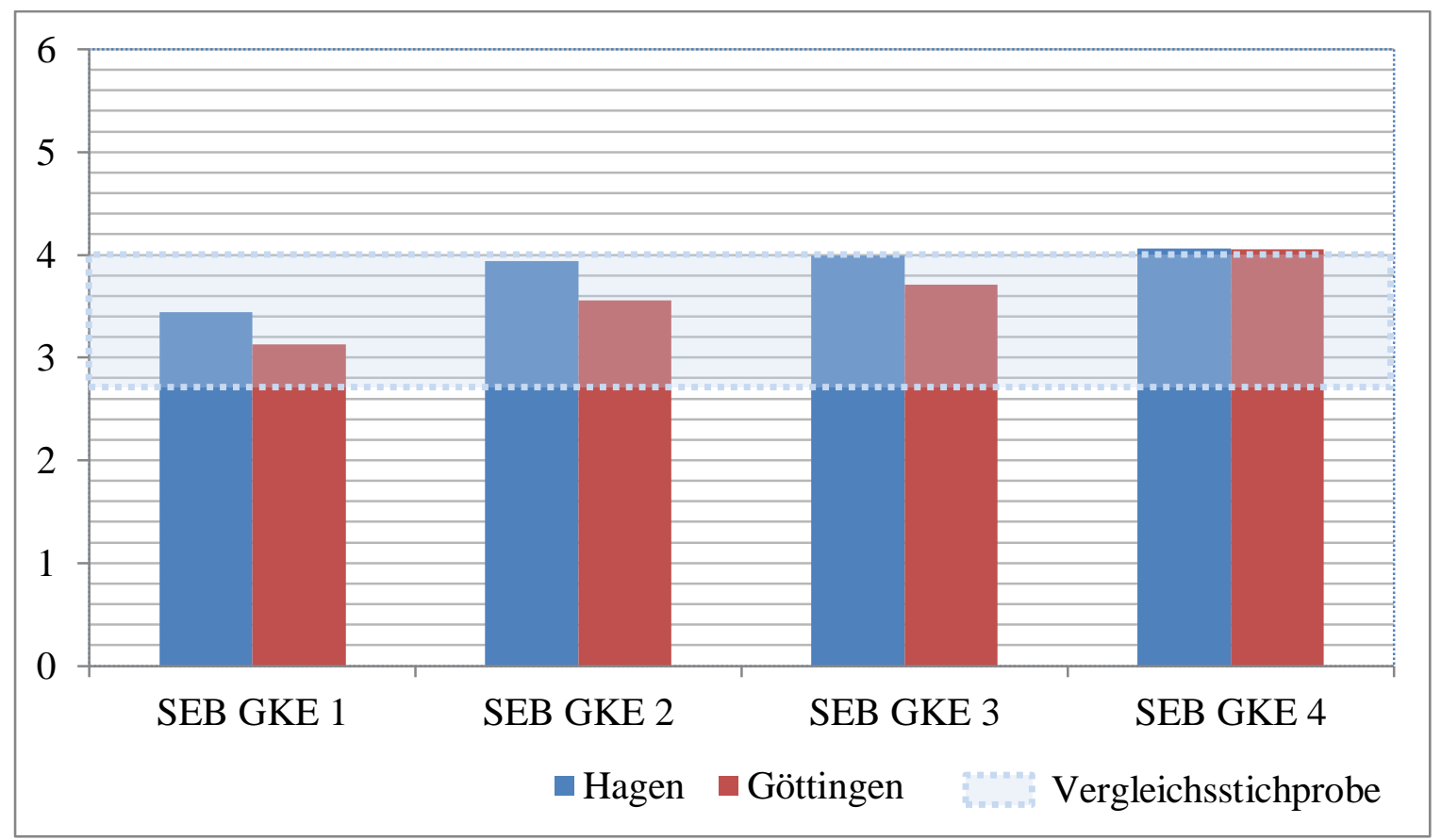

Abb. 16: Stationserfahrungsbogen: Selbstwirksamkeit/Kompetenzerwartung

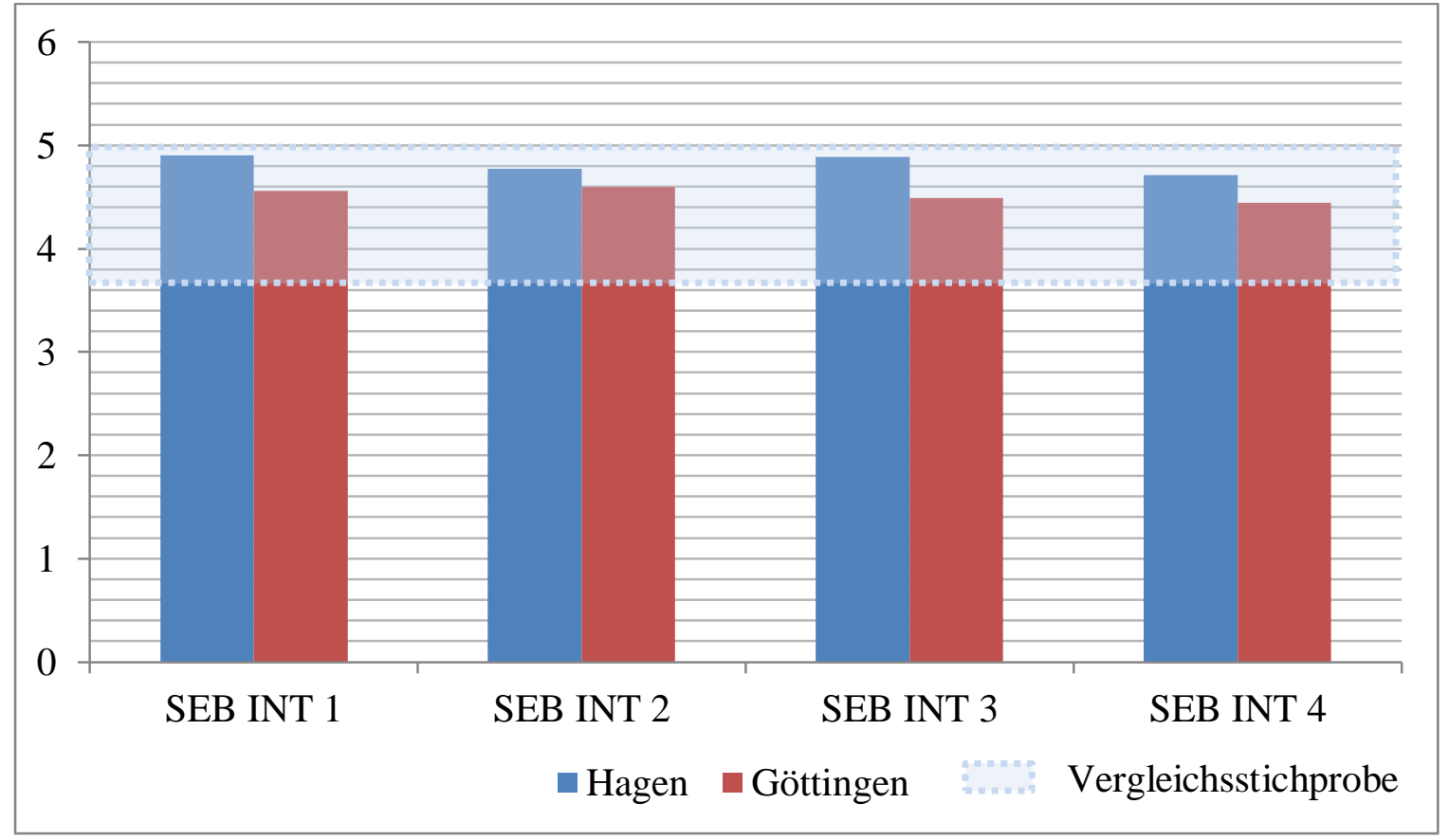

Abb. 17: Stationserfahrungsbogen: Angemessenheit der Behandlungsintensität 


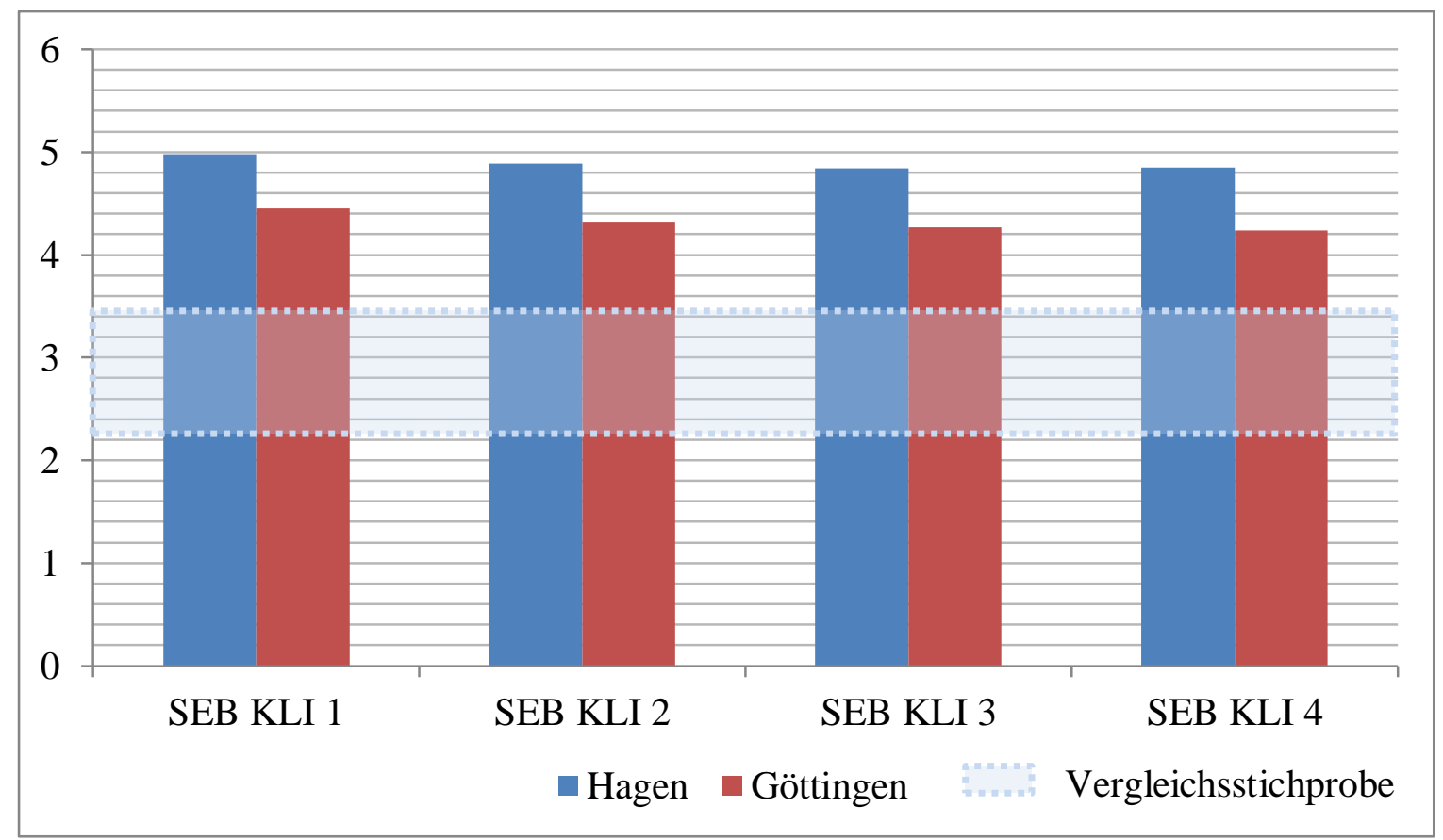

Abb. 18: Stationserfahrungsbogen: Gruppenklima

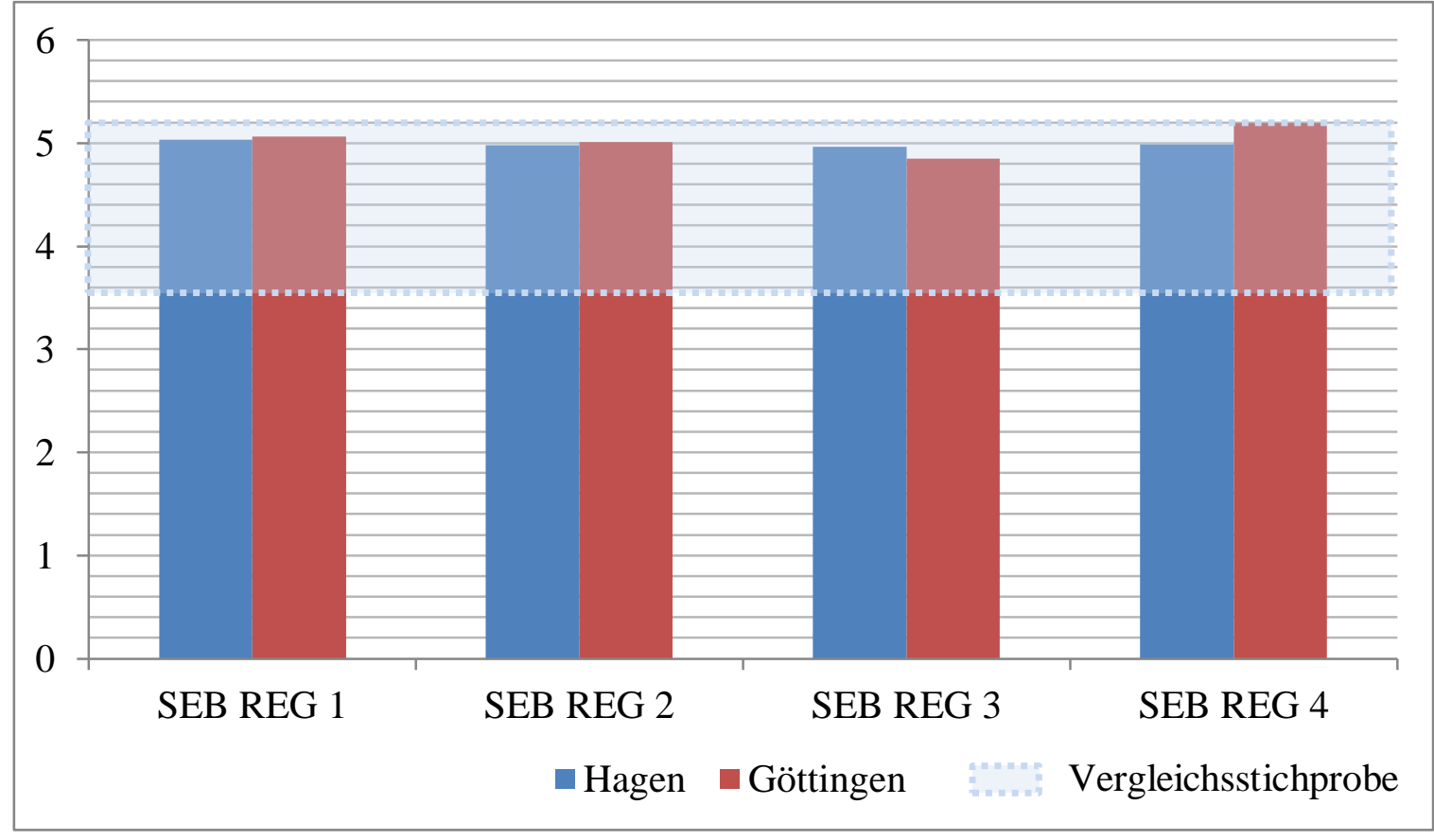

Abb. 19: Stationserfahrungsbogen: Akzeptanz der Rahmenbedingungen 


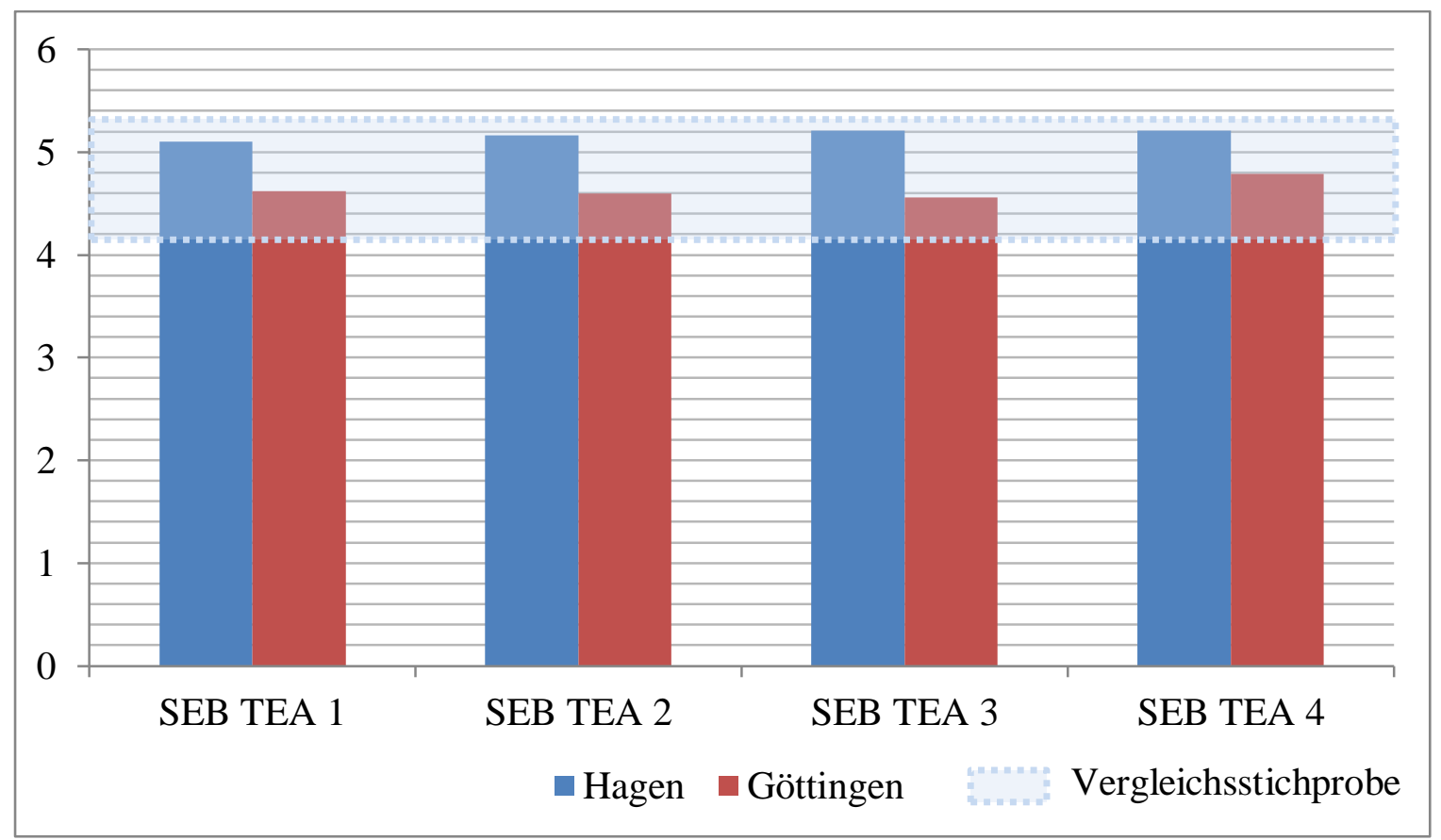

Abb. 20: Stationserfahrungsbogen: Beziehung zum therapeutischen Team

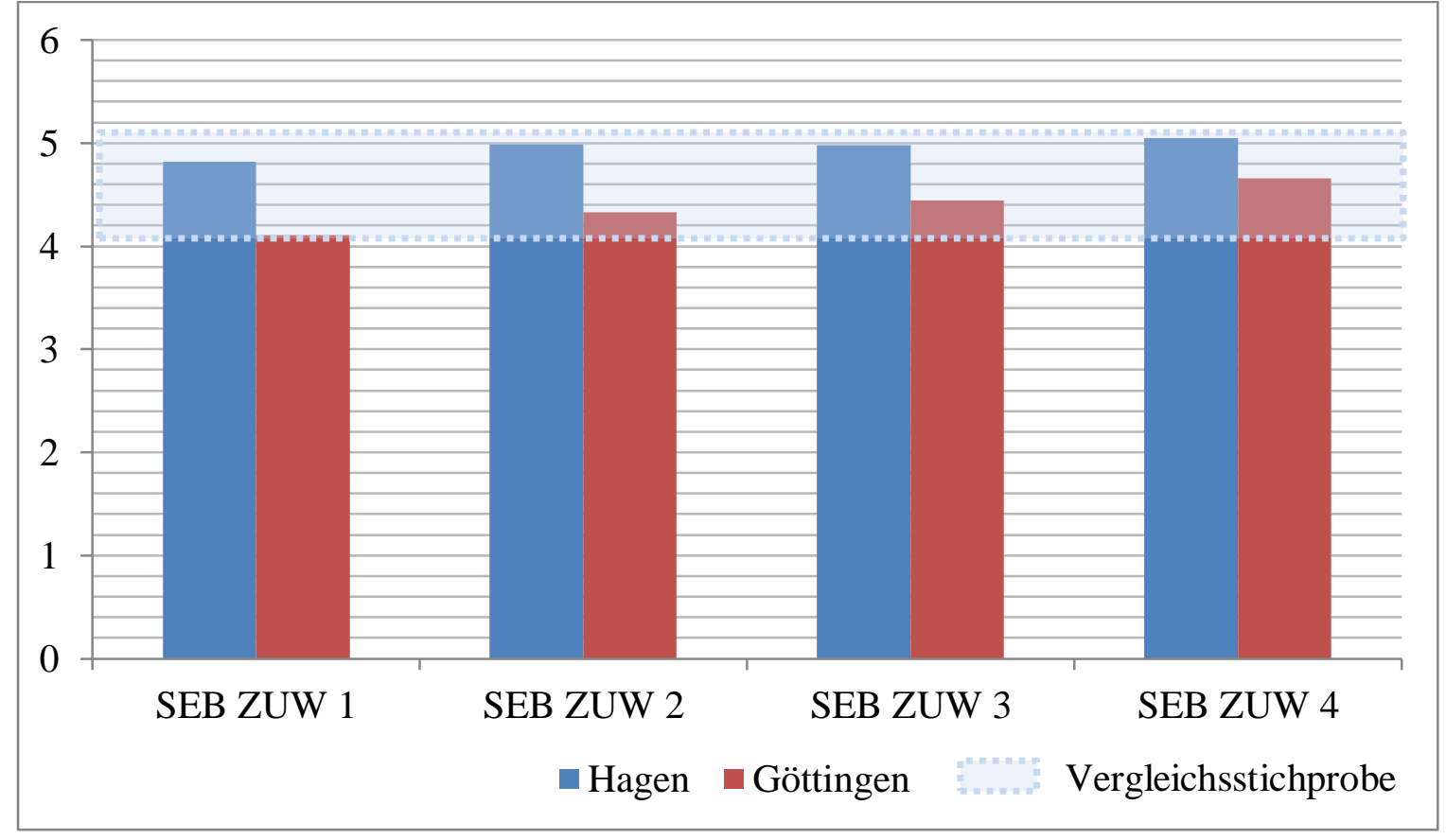

Abb. 21: Stationserfahrungsbogen: Zuwendung durch Mitpatienten 


\title{
5. Diskussion
}

In der vorliegenden Untersuchung wurden die Behandlungsergebnisse von 42 Patienten evaluiert, die auf der psychotherapeutischen Station der Abteilung für Psychiatrie und Psychotherapie der Katholischen Krankenhaus $\mathrm{GmbH}$ in Hagen auf psychodynamischer Grundlage behandelt wurden. Die Behandlungsmethode war psychodynamisch ausgerichtet mit einem Schwerpunkt auf psychoanalytisch-interaktioneller Gruppentherapie, ergänzt von dialektisch-behavioralen Elementen. Diese Ergebnisse wurden verglichen mit den Behandlungsergebnissen von 40 Patienten einer psychotherapeutischen Station des Ask lepiosFachklinikums Göttingen, deren Behandlung an der Dialektisch-Behavioralen Therapie ausgerichtet war, ohne Anwendung psychoanalytisch-interaktioneller Gruppentherapie.

Ziel der Untersuchung war es, die Wirksamkeit psychoanalytisch-interaktioneller Gruppentherapie auf einer psychotherapeutischen Station einer allgemeinpsychiatrischen Klinik der Pflicht- und Regelversorgung unter naturalistischen Bedingungen näher zu evaluieren. Hierzu wurden zwei Hypothesen formuliert, die nachfolgend überprüft werden.

\subsection{Hypothese 1:}

\begin{abstract}
„Es wird angenommen, dass Patienten, die auf einer allgemeinpsychiatrischen Station auf psychodynamischer Grundlage mit psychoanalytisch-interaktioneller Gruppentherapie und Dialektisch-Behavioralen Therapiebausteinen behandelt werden, Verbesserungen ihrer Symptomatik im Verlauf der Behandlung aufweisen.“
\end{abstract}

Diese Hypothese konnte durch die Untersuchungsergebnisse bestätigt werden. In der Hagener Patientengruppe verbesserten sich Symptomschwere, interpersonelle Probleme, Lebenszufriedenheit (aus Patientensicht) und Beeinträchtigungsschwere (aus Therapeutensicht) signifikant und mit mittlerer bis großer Effektstärke.

Die Wirksamkeit psychodynamischer Psychotherapie in der stationären Behandlung ist vielfach belegt (Strauß und Burgmeier-Lohse 1994, Bassler 1995, Junge und Ahrens 1996, Franz et al. 2000, Sack et al. 2003, Rudolf et al. 2004, Bauer et al. 2005, Beutel et al. 2005, Dinger und Schauenburg 2010). Allerdings bezogen sich diese Untersuchungen ausschließlich auf psychosomatische Fachabteilungen, meist an Universitätskliniken. Eine Ausnahme macht die Untersuchung von Franz et al. (2000), eine Multizenterstudie, die sich auf Akutkrankenhäuser bezog, allerdings waren die untersuchten Abteilungen psychosomatisch und nicht allgemeinpsychiatrisch ausgerichtet. $\mathrm{Zu}$ psychotherapeutischen Behandlungsergebnissen in allgemeinpsychiatrischen Kliniken ohne universitären Hintergrund lassen sich keine vergleichbaren Untersuchungen finden. Mit der Wirksamkeit stationärer psychodynamischer Therapie für schwer beeinträchtigte Patienten mit 
Erkrankungen aus dem schizophrenen Formenkreis unter naturalistischen Bedingungen beschäftigten sich Leichsenring et al. (2005) und fanden signifikante Verbesserungen der psychischen Symptomatik.

Untersuchungen zur spezifischen Wirksamkeit psychoanalytisch-interaktioneller (Gruppen-) Therapie zeigten ebenfalls einen positiven Wirkungsnachweis (Davies-Osterkamp et al. 1992, Konzag und Fikentscher 1998, Rabung et al. 2005, Leichsenring et al. 2007, Schellenberg et al. 2004, Salzer et al. 2010). Zwei Studien sind dabei besonders gut mit der aktuellen vergleichbar, da sie ähnliche Testinstrumente verwendeten:

Rabung et al. (2005) evaluierten die Behandlungsergebnisse einer diagnostisch heterogenen Gruppe von Patienten, die mit psychoanalytisch-interaktioneller Gruppentherapie einerseits oder mit tiefenpsychologisch fundierter bzw. analytischer Gruppentherapie andererseits stationär behandelt wurden. Hierbei fanden sich in beiden Gruppen signifikante Verbesserungen der Symptombeeinträchtigung (SCL 90-R), der interpersonellen Probleme (IIP) und der Beeinträchtigungsschwere (BSS). Ein Unterschied zwischen den Gruppen bestand im Ausmaß der Verbesserung interpersoneller Probleme, die in der psychoanalytischinteraktionellen Gruppe deutlicher ausfielen, allerdings betrug die durchschnittliche Behandlungsdauer in dieser Gruppe rund 14 Wochen gegenüber 8 Wochen in der tiefenpsychologisch/analytischen Gruppe, was von den Autoren als entscheidender Faktor angesehen wurde. Ein wesentliches weiteres Ergebnis war, dass sowohl schwerer als auch leichter beeinträchtigte Patienten von der psychoanalytisch-interaktionellen Gruppentherapie gleichermaßen profitierten.

Die vorliegende Untersuchung scheint die Ergebnisse von Rabung et al. (2005) zu bestätigen. Zum einen handelt es sich bei der Hagener Patientengruppe um eine diagnostisch sehr heterogene Gruppe, die im Laufe der Behandlung deutliche Verbesserungen in der Symptomatik und Beeinträchtigungsschwere, aber auch der interpersonellen Probleme aufwies, mit noch etwas deutlicheren Effektstärken. Aufgrund der geringen Stichprobengröße wurden keine Daten erhoben, die speziell Hinweise zur strukturellen Störungsschwere geben würden. Es kann allerdings angenommen werden, dass es sich in dieser unausgelesenen Stichprobe um ein heterogenes Muster auch in den strukturellen Merkmalen handelt. Hervorzuheben ist insbesondere die deutliche Verbesserung der interpersonellen Probleme, da die Behandlungsdauer hier mit 7,6 Wochen vergleichbar ist mit der tiefenpsychologisch/analytischen Gruppe von Rabung et al. (2005). Dieser Befund steht im Widerspruch zu der Annahme von Rabung et al. (2005), dass die Verbesserung in diesem Bereich überwiegend durch die längere Behandlungsdauer in der psychoanalytischinteraktionellen Gruppe verursacht wurde. Vielmehr könnte man dieses Ergebnis als Hinweis auf eine spezifischere Beeinflussung interpersoneller Probleme durch die psychoanalytischinteraktionelle Gruppentherapie verstehen.

In einer naturalistischen Studie untersuchten Leichsenring et al. (2007) die Wirksamkeit stationärer psychoanalytisch-interaktioneller Therapie bei Borderline-Patienten und konnten signifikante Verbesserungen in den hier relevanten Items Symptomschwere (SCL-90-R), Lebenszufriedenheit (FLZ), interpersonelle Probleme (IIP) und Beeinträchtigungsschwere (BSS) mit mittleren bis großen Effektstärken nachweisen. 
Die Ergebnisse der vorliegenden Untersuchung sind mit der Studie von Leichsenring et al. (2007) gut vergleichbar und weisen einige Parallelen auf ${ }^{10}$. Die Effektstärken in der Verbesserung der Lebenszufriedenheit waren nahezu identisch, die interpersonellen Probleme verbesserten sich ähnlich stark. Die Symptomschwere nahm in beiden Studien ab, in der aktuellen allerdings stärker im Vergleich zu Leichsenring et al. (2007).

Die Gesamt-Beeinträchtigungsschwere war sowohl bei Leichsenring et al. (2007) als auch in der aktuellen Studie in der Hagener Patientengruppe sehr hoch und besserte sich in beiden Studien deutlich. Dennoch war die Beeinträchtigungsschwere in der Untersuchung von Leichsenring et al. (2007) zum Entlassungszeitpunkt noch deutlich ausgeprägt, wohingegen die Patienten in Hagen zum Behandlungsende durchschnittlich als leicht beeinträchtigt beurteilt wurden. Die außerordentlich hohe Effektstärke in der Reduktion der Beeinträchtigungsschwere in Hagen ist ein Ergebnis, das sich auch deutlich von der Göttinger Patientengruppe unterscheidet und bei der Erörterung der 2. Hypothese differenzierter betrachtet wird (vgl. Kapitel 5.4.4, Seite 73). Hier sei zunächst festzuhalten, dass sowohl in der Studie von Leichsenring et al. (2007) als auch in der vorliegenden Untersuchung die psychische Beeinträchtigung die ausgeprägteste Verbesserung aufwies, gefolgt von der sozialkommunikativen.

Ein besonderes Augenmerk sei an dieser Stelle nochmals auf die Verbesserung der interpersonellen Probleme gerichtet. Diese verbesserten sich sowohl in der Untersuchung von Leichsenring et al. (2007) als auch in der Hagener Patientengruppe, allerdings hier bei deutlich kürzerer Behandlungsdauer. Viele Forschungsergebnisse weisen darauf hin, dass es in der Regel erst mit einer gewissen Zeitverzögerung zur Veränderung interpersoneller Probleme im Rahmen einer stationären Psychotherapie kommt, sich diese aber stattdessen auch nach der Entlassung nochmals verbessern (Strauß und Burgmeier-Lohse 1994, Bauer et al. 2005, Franke et al. 2005). Die Reduktion interpersoneller Schwierigkeiten in der Hagener Gruppe könnte teilweise Folge der deutlichen Symptomverbesserung sein, die sich während der Behandlung einstellte, da beides miteinander korreliert (Sammet et al. 2004, CritsChristoph et al. 2005). Die relativ rasche Veränderung im interpersonellen Bereich könnte gleichzeitig auch als Hinweis gewertet werden, dass diese besondere Berücksichtigung in der psychoanalytisch-interaktionellen Gruppentherapie gefunden haben.

In diesem Zusammenhang fällt ein interessantes Detail in der Verbesserung der SCL auf: Das Item „Unsicherheit im Sozialkontakt“", das wohl die deutlichste interpersonale Komponente in der SCL aufweist, war in beiden erwähnten Vergleichsstudien von Rabung et al. (2005) und Leichsenring et al. (2007) sowie der vorliegenden unter den drei am deutlichsten verbesserten Items zu finden. Die SCL wurde auch in anderen Untersuchungen zur Wirksamkeit stationärer psychodynamischer Psychotherapie angewendet, so z.B. bei Franz et al. (2000), Konzag et al. (2000) und Franke et al. (2005). Obwohl auch hier insgesamt eine deutliche Symptomverbesserung eintrat, war die Unsicherheit im sozialen Kontakt hier nicht unter den

\footnotetext{
${ }^{10}$ Einschränkungen der Vergleichbarkeit betreffen die Behandlungsdauer, die bei Leichsenring et al. (2007) durchschnittlich 12,5 Wochen betrug und somit nahezu doppelt so lang war wie in der Hagener Gruppe. Des Weiteren bezog sich die Untersuchung auf Einzel- und Gruppentherapie, so dass sich die Ergebnisse nicht allein auf gruppentherapeutische Wirkfaktoren zurückführen lassen, wohl aber auf die psychoanalytisch-interaktionelle Methode.
} 
am stärksten verbesserten Items. Leider lassen sich nur wenig Details zur psychotherapeutischen Behandlung, insbesondere zu Art, Anwendung und Ausmaß von Gruppentherapie aus den Studien entnehmen. Trotzdem scheint dieser Aspekt die Vermutung zu unterstützen, dass sich die Wirkung psychoanalytisch-interaktioneller Therapie insbesondere in der Verbesserung interpersoneller Schwierigkeiten und somit auch sozialer Unsicherheit zeigt.

Der Einfluss der psychoanalytisch-interaktionellen Gruppentherapie auf die Therapieergebnisse in der Hagener Gruppe lässt sich nicht direkt aus der Studie ableiten. Hierzu wäre zumindest eine Kontrollgruppe erforderlich, die unter identischen Bedingungen behandelt wird, ohne Gruppentherapie. Diese Bedingung wäre auf einer Station mit 16 Betten nicht durchzuführen, schon allein durch die geringe Größe der Untergruppen. Darüber hinaus wäre das Ziel der Studie, eben gerade die Wirkung unter naturalistischen Bedingungen zu untersuchen, damit verfehlt worden.

Die Kontrolle des Einflusses begleitender Therapien stellt in der stationären Gruppentherapieforschung ein bekanntes Problem dar und konnte bisher nicht gelöst werden, wie beispielsweise Kösters und Strauß (2007) in einer großen Metaanalyse zur Wirksamkeit stationärer Gruppentherapie feststellten. Sie gehen dennoch davon aus, dass trotz des multimodalen Vorgehens auf Psychotherapiestationen die ,wesentlichen Veränderungsprozesse sich in der Gruppenpsychotherapie fokussieren und dort bearbeitet werden können, (...) (S.183). Da die gesamte Therapiedosis der psychoanalytischinteraktionellen Gruppentherapie in Hagen die anderer Therapien auf der Station deutlich übertraf, darf postuliert werden, dass der Einfluss auf das Gesamtbehandlungsergebnis groß war.

Die Kombination psychoanalytisch-interaktioneller Therapie mit dialektisch-behavioralen Elementen wurde in der Hagener Klinik bewusst gewählt in der Hoffnung, additive oder synergistische Effekte zu erzielen, was auch dem allgemeinen klinischen Eindruck entspricht, wenngleich hier eindeutige empirische Untersuchungen fehlen (Linehan 1996a, Stiglmayr et al. 2002). Um spezifische Wirkungen der psychoanalytisch-interaktionellen Gruppentherapie herauszufiltern, bot sich eine Vergleichsgruppe an, die ausschließlich auf DialektischBehaviorale Therapie zurückgreift, wie es in der Göttinger Klinik der Fall ist. Dennoch können synergistische Effekte damit natürlich nicht ausgeschlossen werden

Für den Vergleich der Behandlungsergebnisse und um die Wirkung psychoanalytischinteraktioneller Gruppentherapie näher einzugrenzen, wurde eine zweite Hypothese formuliert: 


\subsection{Hypothese 2:}

„Es wird angenommen, dass Patienten, die mit Dialektisch-Behavioraler Therapie allein behandelt werden, ebenfalls Verbesserungen bezüglich ihrer Symptome zeigen, sich diese Behandlungsergebnisse aber unterscheiden von denen der Patienten, die zusätzlich an psychoanalytisch-interaktioneller Gruppentherapie teilnehmen.“

Auch diese Hypothese konnte durch die Untersuchung bestätigt werden. Die wesentlichen Ergebnisse seien kurz zusammengefasst:

1. In beiden Kliniken fanden sich zwischen Aufnahme und Entlassung signifikante Verbesserungen in den Bereichen Symptombelastung, Lebenszufriedenheit und Beeinträchtigungsschwere.

2. In der Klinik Hagen waren die Ergebnisse in allen Testbereichen signifikant verbessert und von mittlerer bis hoher Effektstärke.

3. In der Klinik Göttingen zeigten sich signifikante Verbesserungen in den Bereichen Symptombelastung, Lebenszufriedenheit und Beeinträchtigungsschwere, die Effektstärke war hier ebenfalls mittel bis hoch. Für den Bereich der interpersonellen Probleme fand sich hingegen keine signifikante Verbesserung.

4. Im direkten Vergleich waren die Ergebnisse der Klinik Hagen in zwei Bereichen (Befindlichkeitsschwere und interpersonelle Probleme) signifikant besser als in der Klinik Göttingen. Für den Bereich der Symptombelastung zeigten sich in Hagen deutlich größere Effektstärken, der Unterschied verfehlte aber knapp das Signifikanzniveau. In der Lebenszufriedenheit unterschieden sich die Ergebnisse zwischen den Kliniken nicht signifikant.

5. Für den Bereich der Stationserfahrungen waren die Ergebnisse nur eingeschränkt verwertbar, hier zeigten sich in beiden Kliniken ähnliche Ergebnisse ohne deutliche Veränderung im Behandlungsverlauf und entsprachen im Wesentlichen der Vergleichsgruppe von Sammet und Schauenburg (1999).

\subsection{Vergleichbarkeit der Stichproben}

\subsubsection{Soziodemografische Daten}

Die Stichproben waren bezüglich Stichprobenumfang, Geschlechterverteilung und Schulbildung gut vergleichbar. Bezüglich des Alters ergab sich hingegen ein signifikanter Unterschied mit einem höheren Altersdurchschnitt in der Hagener Gruppe (vgl. Kapitel 4.2.4, Abbildung 12 auf Seite 43). Dies ist zum einen durch zwei Hagener Patienten zu erklären, die 
älter als 50 Jahre waren, was bei der untersuchten Stichprobengröße bereits deutlich ins Gewicht fällt. Demgegenüber war in der Göttinger Gruppe die jüngste Altersgruppe im Alter bis 29 Jahre am stärksten vertreten und entspricht damit anderen Klientelen in stationärer Dialektisch-Behavioraler Therapie (Bohus et al. 2000, Bohus et al. 2004, Kröger et al. 2006).

Zum Einfluss des Alters auf psychotherapeutische Behandlung gibt es unterschiedliche Befunde: Riedel (1991) fand in seiner Untersuchung zu verschiedenen Patientenmerkmalen als Determinanten für Therapieerfolg, „daß Patienten mittleren Alters eine signifikant günstigere Beschwerdeverringerung aufweisen als Jüngere und Ältere" (Riedel 1991, S.24) ${ }^{11}$. Allerdings wurde diese Altersklasse im Beeinträchtigungs-Schwere-Score auch von den Therapeuten als geringer beeinträchtigt eingeschätzt. Demgegenüber konnten Franz et al. (2000) keinen nennenswerten Einfluss des Alters auf das Therapieergebnis in ihrer Multizenterstudie feststellen. Wolf et al. (2011) untersuchten den Therapieerfolg von über 60jährigen, die stationär psychotherapeutisch behandelt wurden, und fanden sowohl zum Entlassungszeitpunkt als auch in der Katamnese gute Erfolge mit hohen Effektstärken. Sie schlossen daraus, dass auch ältere Patienten von regulär durchgeführter stationärer Psychotherapie gut profitieren können, ohne dass eine Modifikation der Methoden erforderlich ist. Gunzelmann et al. (2000) fanden in einer nicht-klinischen Population keine Unterschiede in den interpersonalen Problemen bei über 60-jährigen, gemessen am IIP.

Bezogen auf diese Forschungsergebnisse dürfte der höhere Altersdurchschnitt in der Hagener Gruppe somit kaum zu einer systematischen Beeinflussung der Testergebnisse in der aktuellen Untersuchung beigetragen haben.

\subsubsection{Diagnosen}

Zwischen den beiden Patientengruppen gab es deutliche Unterschiede in der Variabilität und Verteilung der psychiatrischen Diagnosen. Hierbei war trotz identischer Komorbiditätsrate in beiden Kliniken in Hagen ein größeres diagnostisches Spektrum vertreten, das neben den Schwerpunktdiagnosen aus dem depressiven Bereich und den Persönlichkeitsstörungen auch Suchtmittelkonsum und somatoforme Störungen einschloss. In Göttingen hingegen waren die Diagnosen homogener verteilt, neben einer Achse-I-Störung wies jeder Patient eine Persönlichkeitsstörung, überwiegend vom Subtyp „emotional-instabil“ auf; Suchtmittelgebrauch wurde nicht diagnostiziert (vgl. hierzu Kapitel 4.3.1, Abbildung 13 auf Seite 46).

Diese Unterschiede bezüglich der diagnostischen Verteilung lassen sich mit den unterschiedlichen institutionellen Gegebenheiten (Rahmenbedingungen erster Ordnung, Janssen 2004) erklären.

Die Hagener Klinik ist eine allgemeinpsychiatrische Akutklinik und hat nur eine Station für alle psychotherapeutisch zu behandelnden Patienten zur Verfügung. Dementsprechend ist die Spezifität der behandelten Diagnosen gering. Durch die Integration in ein großes

\footnotetext{
${ }^{11}$ Die Kategorie „,mittleres Alter“ entsprach hier der Altersklasse zwischen 25 und 35 Jahren, „Jüngere“ waren $<25$ Jahre, „Ältere“>35 Jahre alt (Anm. d. Verf.)
} 
konfessionelles Allgemeinkrankenhaus besteht räumliche und fachliche Nähe zu vielen somatischen Disziplinen, somatische Komorbidität ist hier für die Aufnahme kein Hinderungsgrund.

Die Göttinger Klinik hingegen ist eine große psychiatrisch-psychotherapeutische Fachklinik. Hier findet bereits eine Vorselektion bezüglich der Diagnosen vor Einweisung oder Verlegung der Patienten statt. Dies wirkt sich auf die Spezialisierung aus und zeigt sich daran, dass allein drei Psychotherapiestationen mit unterschiedlichen Behandlungsschwerpunkten und enger Kooperation bestehen (vgl. hierzu auch Herbold und Sachsse 2008). Es ist erwartbar, dass auf einer Station mit einem eher störungsspezifisch angelegten dialektischbehavioralen Behandlungssetting überwiegend Patienten mit einer Persönlichkeitsstörung aus dem emotional-instabilen Bereich behandelt werden, wie dies auch der Fall war.

Die Unterschiede in den Diagnosen können systematischen Einfluss auf die Behandlungsergebnisse haben. Der signifikant größere Anteil von Patienten mit Persönlichkeitsstörungen in Göttingen könnte sich dabei negativ auf die Behandlungsergebnisse ausgewirkt haben, da diese Patienten mehr interpersonelle und intrapersonelle Probleme aufweisen (Wuchner et al. 1993), mit einem schlechteren Behandlungsergebnis korreliert sind (Bassler 1995) und in der Regel länger behandelt werden (Zielke et al. 1997, Beutel et al. 2005). Demgegenüber steht allerdings die hohe Störungsspezifitiät der Behandlung mit Dialektisch-Behavioraler Therapie und nachgewiesenen guten Behandlungserfolgen gerade bei der Patientengruppe der BorderlinePersönlichkeitsstörung, sowohl ambulant (Linehan et al. 1991, Linehan et al. 1994, Linehan et al. 1999, Turner 2000, Koons et al. 2001, Linehan et al. 2006) als auch stationär (Bohus et al. 2000, Bohus et al. 2004), hier auch bei Komorbidität mit anderen Störungsbildern (Kröger et al. 2006).

Der geringere Anteil persönlichkeitsgestörter Patienten in Hagen könnte das Behandlungsergebnis positiv beeinflusst haben. Allerdings wurden für die psychoanalytischinteraktionelle Therapie gerade bezüglich dieser Patientengruppe gute Ergebnisse gefunden (Rabung et al. 2005, Leichsenring et al. 2007). Die größere Variabilität der Diagnosen stellt außerdem ebenfalls eine hohe Anforderung an die psychotherapeutische Behandlung, da einerseits die individuellen Störungscharakteristika berücksichtigt werden müssen, andererseits eine Integration in das stationäre Therapiesetting erfolgen muss. Rudolf et al. (2004) fanden in ihrer Untersuchung zu störungsbezogenen Therapieergebnissen nach stationärer psychodynamischer Psychotherapie, dass weniger die schweren Persönlichkeitsstörungen als vielmehr die somatoformen Störungen, die auch einige Hagener Patienten aufwiesen, mit einem schlechten Therapieergebnis korreliert waren.

Es darf somit vermutet werden, dass der mögliche Vorteil eines geringeren Anteils an Persönlichkeitsstörungen in Hagen durch die größere Variabilität der Störungsbilder relativiert wird. 


\subsubsection{Behandlungsdauer}

Die Behandlungsdauer war mit 7,58 Wochen in der Hagener Stichprobe signifikant länger als in der Göttinger Patientengruppe mit 5,45 Wochen (vgl. Kapitel 4.3.2, Abbildung 14 auf Seite 47). Dies kann durch verschiedene Faktoren verursacht worden sein.

Zunächst ist die Differenz vermutlich begründet in den unterschiedlichen Therapieverfahren. Die Teilnahme an der Gruppentherapie in Hagen bedeutet in der Regel eine mindestens vierbis sechswöchige Behandlungsdauer, um einen Gruppenprozess zu unterstützen. Andere Therapiestudien $\mathrm{zu}$ psychodynamischer stationärer Therapie wiesen tendenziell längere Behandlungszeiten auf, diese lagen zwischen 3-6 Monaten in älteren (Kordy et al. 1990, Strauß und Burgmeier-Lohse 1994, Bassler et al. 1995) und 7-10 Wochen (Junge und Ahrens 1996, Konzag et al. 2000, Franke et al. 2005) in jüngeren Studien.

Die Therapiedauer in Göttingen ist im Vergleich $\mathrm{zu}$ anderen Studien $\mathrm{zu}$ stationärer Dialektisch-Behavioraler Therapie ebenfalls eher kurz. Die stationären Behandlungen sind meist auf ein 12-wöchiges Programm angelegt und die Aufenthaltsdauer entsprechend zwischen 6 und 13 Wochen (Bohus et al. 2000, Bohus et al. 2004, Kröger et al. 2006). Die kürzeren Behandlungszeiten in Hagen und Göttingen im Vergleich zu den zitierten Studien können wiederum mit den institutionellen Gegebenheiten zusammenhängen, da die Behandlungszeiten in psychiatrischen Kliniken im Vergleich z.B. zu psychosomatischen Abteilungen kürzer sind (Freyberger und Spitzer 2012).

Ein weiterer Grund ist vermutlich in den unterschiedlichen Aufnahmebedingungen zu sehen. In der Göttinger Gruppe war die Indikation zur psychotherapeutischen Behandlung schon bei Aufnahme klar. In der Hagener Klinik war die Ausgangslage zum Aufnahmezeitpunkt unübersichtlicher und die Indikationsstellung für eine psychotherapeutische Behandlung noch nicht bei allen Patienten erfolgt, was die Behandlungsdauer insgesamt verlängert.

Weitere Einflussfaktoren, wie z.B. die stärkere körperliche Beeinträchtigung der Hagener Patienten entsprechend der Therapeuteneinschätzung im BSS, können nicht belegt werden. In einer Untersuchung von Borgart und Meermann (1999) spielt beispielsweise die somatische Komorbidität keine Rolle für die Behandlungsdauer. Ein höheres Lebensalter (entsprechend der Hagener Gruppe) führt eher zu kürzerer Behandlungsdauer (Wagner 1996, Borgart und Meermann 1999) und erklärt die Differenz daher nicht. Die Dauer einer stationären psychotherapeutischen Behandlung hat außerdem vielfältige Zusammenhänge mit sozialen Faktoren, z.B. dem Bestehen einer Partnerschaft, die in der aktuellen Studie nicht erhoben wurden und somit auch nicht bewertet werden können (Bassler et al. 1995, Wagner 1996, Zielke et al. 1997, Borgart und Meermann 1999). Aus den Ergebnissen des Stationserfahrungsbogens lassen sich ebenfalls keine systematischen Einflussfaktoren ableiten.

Neben den Ursachen für die Differenz der Behandlungsdauer ist vor allem interressant, in welcher Weise sich diese auf die Ergebnisse der Untersuchung auswirkt. Die Literatur zu stationärer Psychotherapie bezieht sich allerdings in der Regel auf längere Behandlungszeiten. Einzig Nosper (1999) untersuchte die Behandlungsergebnisse in psychosomatischer Rehabilitation nach drei Wochen und zum Ende der Behandlung. Er konnte zeigen, dass sich 
beispielsweise die Symptombelastung nach drei Wochen besserte, sich dieser Effekt aber bis zum Entlassungszeitpunkt nochmals deutlich steigerte, so dass erst dann von einem klinisch bedeutsamen, längerfristig stabilen Behandlungseffekt ausgegangen werden konnte.

Die Bedeutung der Behandlungsdauer für den Therapieerfolg wird immer wieder diskutiert, einerseits aufgrund ökonomischer Aspekte aus Sicht der Kostenträger, andererseits aus therapeutischer Perspektive. Einige Befunde deuten auf größere Verbesserungen bei längerer Therapiedauer hin, vor allem bei der Behandlung von Persönlichkeitsstörungen (Hoglend 1993, Orlinsky et al. 1994, Leichsenring und Leibing 2003). Bassler et al. (1995) fanden demgegenüber „daß eine längere Behandlungsdauer nicht per se zu einem besseren Behandlungserfolg führt (...)“ (Bassler et al. 1995, S. 167), und plädieren eher für eine Intervalltherapie mit wechselndem Behandlungsfokus. Sie weisen außerdem darauf hin, dass sich die stationäre Behandlungsdauer im Laufe der Jahre reduziert hat. Diese Entwicklung hat sich seitdem weiter fortgesetzt; so ist in der genannten Studie eine „Kurzzeittherapie“ mit einer durchschnittlichen Behandlungsdauer von 11,5 Wochen mit der aktuellen Studie kaum vergleichbar. Neben dem globalen Therapieerfolg wirkt sich die Dauer der Behandlung auf die untersuchten Items auch unterschiedlich aus. So benötigen interpersonelle Probleme zur Veränderung mehr Zeit als beispielweise die Symptomreduktion oder körperliche Symptome (Strauß und Burgmeier-Lohse 1994, Kraft et al. 2003, Bauer et al. 2005, Franke et al. 2005).

Zusammenfassend muss davon ausgegangen werden, dass sich die längere Behandlungsdauer in Hagen positiv auf die Behandlungsergebnisse ausgewirkt hat und $\mathrm{zu}$ einem Teil die deutlicheren Therapieeffekte im Vergleich zu der Göttinger Gruppe erklärt.

\subsection{Vergleich der Testergebnisse}

In beiden Kliniken verbesserten sich die Befunde sowohl in der Selbst- als auch in der Fremdbeurteilung. Beim direkten Vergleich der Mittelwerte der einzelnen Testinstrumente fällt allerdings auf, dass die Hagener Gruppe in den drei Selbstbeurteilungsverfahren zwar nicht signifikant, aber doch deutlich unterhalb der Göttinger Gruppe in die Therapie startete, d.h. sich im Durchschnitt weniger durch Symptome belastet fühlte, weniger interpersonelle Probleme hatte und sich bezüglich ihrer Lebenssituation zufriedener fühlte. Beim Fremdratingverfahren verhielt es sich demgegenüber genau umgekehrt: Hier fand sich in der Hagener Gruppe ein hochsignifikant unterschiedlicher, fast doppelt so hoher Eingangswert wie in der Göttinger, was bedeutet, dass die Hagener Patienten durch die Behandler als deutlich schwerer beeinträchtigt eingeschätzt wurden als die Göttinger.

Dieser Befund legt die Vermutung nahe, dass es den Patienten der Hagener Gruppe, zumindest was ihre Selbstbeurteilung angeht, zum Studienbeginn etwas besser ging als den Göttingern. Hierbei muss berücksichtigt werden, dass für die Hagener Patienten der Zeitpunkt, zu dem sie die Selbstbeurteilungsbögen bearbeiteten, nicht für alle Bögen identisch war mit dem Aufnahmezeitpunkt. Dies galt nur für die SCL-90-R; IIP und FLZ hingegen wurden erst später bearbeitet, wenn eine Indikation zur Teilnahme am Gruppentherapieprogramm gestellt worden war. Es hatte zu dieser Zeit also schon eine 
stabilisierende Behandlung stattgefunden, und die Patienten waren schon für eine gewisse Zeit aus Alltagskonflikten herausgenommen, was für sich genommen bereits therapeutisch wirksam ist (Linden et al. 2006).

Die Fremdeinschätzung der Beeinträchtigungsschwere wurde hingegen in beiden Kliniken auf den Aufnahmezeitpunkt bezogen, so dass die Diskrepanz zwischen Selbst- und Fremdbewertung in der Hagener Gruppe hierdurch eine gewisse Erklärung findet.

\subsubsection{Symptombelastung}

Die allgemeine Symptombelastung bei Aufnahme, gemessen am GSI, war hoch, lag in beiden Gruppen etwas über dem von Franke (2002) in der Normstichprobe für stationäre Psychotherapiepatienten ermittelten Wert und auch über dem von Wuchner et al. (1993) erhobenen Wert für Persönlichkeitsstörungen. Dabei war die Symptombelastung in Hagen etwas geringer ausgeprägt als in der Göttinger Gruppe.

Obwohl sich in beiden untersuchten Gruppen signifikante Verbesserungen der Symptombelastung einstellten, waren die Effekte in Hagen wesentlich deutlicher als in Göttingen, gemessen am GSI waren sie nahezu doppelt so groß (vgl. Kapitel 4.4.1, Seite 47). Dies bedeutet, dass die Hagener Patienten zum Entlassungszeitpunkt wesentlich weniger durch Symptome belastet waren als die Göttinger Patienten.

Bei der Erörterung dieses Aspektes muss einschränkend darauf hingewiesen werden, dass in der Varianzanalyse für den Einflussfaktor Klinik auf dieses Ergebnis eine Signifikanz nicht nachgewiesen werden konnte (vgl. Kapitel 4.4.5, Seite 54). Aus statistischer Sicht spricht dies für die Annahme der Nullhypothese (,Es besteht kein Unterschied im Einfluss der Klinik auf die Verbesserung der Symptombelastung im Vergleich zwischen Aufnahme und Entlassung"). Aufgrund der großen Effektstärkendifferenz und dem nur gering verfehlten Signifikanzniveau ist vermutlich die geringe Stichprobengröße der Grund für das nichtsignifikante Ergebnis, und das Risiko, diesen Befund fälschlicherweise als zufallsbedingt einzuschätzen, erscheint relativ hoch. Somit sollte eine nähere Erörterung dieses Befundes erfolgen, auch wenn eine gewisse Irrtumswahrscheinlichkeit besteht.

Verbesserungen der Symptombelastung ließen sich sowohl für die psychodynamische Therapie (Franz et al. 2000, Konzag 2000, Bauer et al. 2005, Beutel et al. 2005, Rabung et al. 2005, Franke et al. 2005, Leichsenring et al. 2007, Kirchmann et al. 2011) als auch die Dialektisch-Behaviorale Therapie (Bohus et al. 2000, Bohus et al. 2004, Kröger et al. 2006) im stationären Rahmen nachweisen. Der hier erzielte Effekt in der Göttinger Gruppe ist weniger deutlich ausgeprägt als in den genannten Vergleichsstudien zur stationären DBTBehandlung, bei allerdings auch deutlich geringerer Behandlungsdauer (s.o., Kapitel 5.3.3, Seite 67).

$\mathrm{Ob}$ die unterschiedliche Behandlungsdauer als wesentliche Ursache für die Differenz zwischen den Gruppen betrachtet werden kann ist fraglich. Die Abnahme der Symptombelastung scheint bereits früh im Verlauf einer stationären Behandlung einzutreten 
und andere Bereiche im Ausmaß der Veränderung zu übertreffen (Junge und Ahrens 1996). So fanden Franke et al. (2005) in stationärer psychodynamischer Therapie einen großen Effekt innerhalb der ersten vier Wochen, der im weiteren Behandlungsverlauf zwar noch zunahm, dies aber deutlich weniger ausgeprägt. Ein ähnliches Ergebnis zeigte sich bei Beutel et al. (2005): In der Kurzzeittherapie mit einer Behandlungsdauer von 4,5 Wochen nahm die Symptombelastung stärker ab als in der Langzeittherapie. Dies widerlegt natürlich nicht, dass die längere Behandlungsdauer in Hagen Einfluss auf die stärkere Verbesserung der Symptomatik hatte, erklärt aber nicht die ausgeprägte Differenz zur Göttinger Gruppe.

Die differenzierte Betrachtung der einzelnen Symptomkategorien bietet einen möglichen Hinweis für eine Differenz, die Bezug zu den unterschiedlichen Behandlungsverfahren haben könnte: In beiden Gruppen besserte sich die Depressivität am deutlichsten, ein Befund, der sich in vielen Untersuchungen findet (vgl. z. B. Junge und Ahrens 1996, Sack et al. 2003, Leichsenring et al. 2007, Rabung et al. 2005). Der oben bereits erwähnte Bereich „Unsicherheit im Sozialkontakt“" besserte sich hingegen in Hagen mit großer Effektstärke, demgegenüber wurde in der Göttinger Gruppe eine mittlere Effektstärke knapp verfehlt.

Die Veränderungen der Symptombelastung wurden in neueren Forschungsansätzen etwas differenzierter betrachtet. So versuchten Kirchmann et al. (2011) neben den klinischen Diagnosen Cluster zu definieren, die mit gutem oder schlechtem Therapieerfolg (in psychodynamischer Therapie) korreliert waren, und diese auch mit interpersonellen Merkmalen in Beziehung zu setzen. So zeigte sich, dass ein hohes Maß an interpersonellen Schwierigkeiten einen behindernden Einfluss auf den Behandlungserfolg hatte, wenn Patienten dem unsicher-phobischen oder somatisierenden Cluster zugeordnet waren. Eine solch differenzierte Betrachtung der Einflussfaktoren war in dieser Studie nicht möglich. Die etwas deutlicher ausgeprägten interpersonellen Schwierigkeiten in der Göttinger Gruppe könnten allerdings die Symptomverbesserung negativ beeinflusst haben.

Nicht zuletzt kann natürlich auch der Einfluss der unterschiedlichen Therapieverfahren nicht ausgeschlossen werden. Aus den vorliegenden Daten kann man allerdings nicht ableiten, dass die psychoanalytisch-interaktionelle Gruppentherapie oder die Dialektisch-Behaviorale Therapie allein für die Differenz in der Symptomreduktion verantwortlich sind. Allenfalls eine gezieltere Beeinflussung sozialer Unsicherheit durch die psychoanalytisch-interaktionelle Gruppentherapie könnte vermutet werden, da sich dieser Effekt hier erneut zeigt. Ein synergistischer Effekt, der sich aus der Kombination der Verfahren in der Hagener Gruppe ergeben haben kann, ist ebenfalls möglich.

\subsubsection{Interpersonelle Probleme}

Der Bereich der interpersonellen Probleme wies in beiden Kliniken deutliche Unterschiede im Behandlungsverlauf auf; dies war, gemeinsam mit dem Aspekt der Beeinträchtigungsschwere, sicherlich das auffälligste Ergebnis der Untersuchung. Die interpersonellen Probleme zeigten bei den Hagener Patienten eine signifikante Verbesserung von mittlerer Effektstärke, wohingegen diese bei den Göttinger Patienten nur sehr gering blieb, ohne das 
Signifikanzniveau zu erreichen. Hierzu lassen sich unter Berücksichtigung des aktuellen Standes der Psychotherapieforschung verschiedene Überlegungen anstellen.

Die Hagener Gruppe wies zum Aufnahmezeitpunkt eine etwas geringere Belastung durch interpersonelle Probleme auf als die Göttinger (vgl. Kapitel 4.4.2, Seite 50), wobei der Unterschied nicht signifikant war (vgl. Kapitel 4.4.5, Seite 54). Ein Teil dieser Differenz, insoweit sie nicht zufällig war, könnte mit dem Unterschied der diagnostischen Zusammensetzung der beiden Populationen erklärt werden. Wuchner et al. (1993) untersuchten den Zusammenhang zwischen verschiedenen Diagnosegruppen und IIP-Werten und fanden unter anderem ein größeres Ausmaß an interpersonellen Problemen bei Patienten mit Persönlichkeitsstörungen. Patienten mit Abhängigkeitserkrankungen oder psychosomatischen Störungen, wie sie in Hagen vorlagen, wiesen demgegenüber geringere Werte auf.

Die Verbesserungen der interpersonellen Probleme in der Hagener Gruppe, bezogen auf die Subskalen, zeigten deutlichere Effekte in den Bereichen ,introvertiert/sozial vermeidend“, „selbstunsicher/unterwürfig“, „fürsorglich/freundlich“ und „ausnutzbar/nachgiebig“, also Problembereiche, die eher im freundlich-unterwürfigen Interaktionsverhalten angesiedelt sind. Demgegenüber waren die Effekte in den feindselig-konkurrierenden Mustern ,autokratisch/dominant“, „streitsüchtig/konkurrierend“ und „abweisend/kalt" deutlich schwächer. In der Göttinger Gruppe zeigte sich der einzig signifikante Effekt ebenfalls im Bereich ,ausnutzbar-nachgiebig“.

Dieses Veränderungsmuster interpersoneller Probleme im Laufe einer psychotherapeutischen Behandlung ist in vielen Untersuchungen belegt worden. Probleme aus dem freundlichsubmissiven Bereich sind, wie in der vorliegenden Studie, zu Beginn stärker ausgeprägt, sind am häufigsten Inhalt in der Therapie und zeigen die größten Veränderungen. Demgegenüber verändern sich Probleme aus dem autokratisch-dominanten Bereich, ähnlich wie Probleme mit Intimität, weniger (Horowitz et al. 1988, Franz et al. 2000, Liedtke und Geiser 2001, Crits-Christoph et al. 2005).

Der positive Einfluss psychodynamischer Gruppentherapie auf die Veränderung interpersoneller Probleme wurde in verschiedenen Studien nachgewiesen, so z.B. von Liedtke und Geiser (2001) und Rabung et al. (2005, s.o.). Für stationäre Dialektisch-Behaviorale Therapie wurde in den vorliegenden Studien dieser Aspekt nicht untersucht. Linehan et al. (1994) fanden Verbesserungen interpersoneller Schwierigkeiten durch ambulante DBTBehandlung bei Borderline-Patientinnen.

Bezogen auf die zitierten Forschungsergebnisse kann die Verbesserung interpersoneller Probleme in Hagen durchaus mit der Behandlung durch psychoanalytisch-interaktionelle Gruppentherapie in Verbindung gebracht werden, wie bereits weiter oben (s. Kapitel 5.1, S. 60) dargelegt wurde. Es gibt allerdings auch andere mögliche Einflussfaktoren, die zu diesem Unterschied im Vergleich zur Göttinger Gruppe beigetragen haben könnten: So tritt z.B. die Verbesserung interpersoneller Probleme zeitverzögert auf und geht nicht mit der Verbesserung der Symptome parallel (Franz et al. 2000, Liedtke und Geiser 2001, Huber et al. 
2007). Insofern könnte die längere Behandlungsdauer in Hagen insbesondere in Bezug auf dieses Item Relevanz haben.

Dass die initial stärkere Ausprägung interpersoneller Probleme in Göttingen ein Hindernis für die Verbesserung im Verlauf darstellt, lässt sich demgegenüber nicht auf der Grundlage der vorliegenden Literatur begründen. In mehreren Studien waren initial höhere Werte für interpersonelle Probleme mit einer höheren Effektstärke verbunden (Mohr et al. 1990, Liedtke und Geiser 2001). Hierbei scheinen andere Faktoren wie das subjektive Leiden und vermutlich auch die Differenzierung nach Subgruppen eine Rolle zu spielen. Liedtke und Geiser (2001) fanden beispielsweise, dass Patienten mit hohen Werten für Autokratie und Submissivität ein besonders gutes Therapieergebnis erreichten. Für eine differenziertere Betrachtung von Subgruppen im Bereich der IIP, bezogen auf die klinische Relevanz der Veränderung, plädieren auch Salzer et al. (2010), ein Aspekt, der für zukünftige Studien interessant und zu berücksichtigen wäre.

\subsubsection{Lebenszufriedenheit}

Die Befunde zur Lebenszufriedenheit waren am wenigsten eindeutig, was allerdings zu erwarten war. Dieser Teil der Untersuchung wurde nicht mit dem Anspruch hinzugefügt, differenzierte klinisch-psychotherapeutische Zusammenhänge zwischen der Behandlung und der Lebenszufriedenheit aufzuzeigen, sondern sollte eher einen Überblick verschaffen, ob sich auch dieser Aspekt im Laufe der Behandlung verändert. Daher wurde auch nur eine Kurzform des Fragebogens angewendet.

Die Ergebnisse zeigten die geringsten Differenzen zwischen den beiden untersuchten Patientengruppen. Die Hagener waren gegenüber den Göttinger Studienteilnehmern zu Beginn der Behandlung zufriedener. In beiden Gruppen besserte sich die Lebenszufriedenheit der Patienten im Verlauf signifikant, in der Göttinger Patientengruppe war diese Verbesserung etwas deutlicher als bei den Hagener Patienten (vgl. Kapitel 4.4.3, Seite 52) Der Unterschied war allerdings nicht signifikant und der Zwischengruppeneffekt nur gering (vgl. Kapitel 4.4.5, Seite 54).

Der Begriff der Lebenszufriedenheit ist sehr heterogen und hängt von sehr vielen Faktoren ab, wie bereits in der Beschreibung des Testinstruments FLZ (Fahrenberg et al. 2000) erörtert wurde. Die erhobenen Items beziehen sich überwiegend auf Lebensbereiche, zu denen keine vergleichbaren Daten erhoben wurden, wie z. B. Partnerschaft oder sozioökonomischer Status. Hierzu können also keine näheren Aussagen getroffen werden, auch nicht, inwieweit sich diese Bereiche unabhängig von der Behandlung geändert haben. Andererseits kann vermutet werden, dass die deutliche Verbesserung der Lebensqualität in beiden untersuchten Stichproben nicht allein durch von der Behandlung unabhängige Faktoren bedingt war. Wo könnten also Zusammenhänge bestehen?

Nach Fahrenberg et al. (2000) ist „Der Gesundheitszustand (...) vermutlich der wichtigste Faktor der allgemeinen Lebenszufriedenheit" (zitiert nach Fahrenberg et al. 2000, S. 16). In der vorliegenden Studie zeigten Patienten in beiden Gruppen signifikante Verbesserungen in 
der Symptombelastung und der Beeinträchtigungsschwere. Nimmt man diese Instrumente als $\mathrm{Maß}$ für den Gesundheitszustand, wäre hier ein möglicher Zusammenhang zwischen der Verbesserung der Lebensqualität und der Behandlung festzustellen.

Mehrere Studien fanden eine negative Korrelation zwischen Depression und Lebenszufriedenheit (Newsom und Schulz 1996, Silveira und Ebrahim 1998) sowie eine positive Korrelation zwischen Extraversion und Lebenszufriedenheit (DeNeve und Cooper 1998). In der vorliegenden Studie zeigte sich in beiden Gruppen eine signifikante Besserung vor allem der Skala „Depressivität“ in der SCL, so dass bei negativer Korrelation hiermit eine Verbesserung der Lebensqualität einhergehen müsste. Auch hier kann also ein Zusammenhang vermutet werden. In Bezug auf den Faktor Extraversion fällt auf, dass sich vor allem in der Hagener Gruppe eine Verbesserung der interpersonellen Probleme, hier insbesondere bezogen auf die Subskala Introversion, ergeben hat. Möglicherweise hat dieses Ergebnis auch zur Verbesserung der Lebensqualität beigetragen. Für die Göttinger Patienten ließ sich dieser Effekt in Bezug auf die interpersonellen Probleme nicht nachvollziehen.

\subsubsection{Beeinträchtigungsschwere}

Betrachtet man die Absolutwerte der Fremdraterskala, findet sich hier zum Zeitpunkt der Aufnahme der Patienten in Hagen ein durchschnittlicher Summenwert von 7,9, dies entspricht einer ausgeprägten bis außerordentlich schweren Erkrankung (vgl. hierzu Kapitel 3.2.4, Seite 34 und Kapitel 4.4.4, Seite 52). Dieser Schweregrad stimmt mit der von Schepank (1995) aufgeführten Stichprobe stationärer psychotherapeutischer Patienten überein, zeigt aber schon eine Verlagerung in den Bereich einer starken Beeinträchtigung. Auch in anderen Studien, in denen der BSS angewendet wurde, entsprach die Beeinträchtigung bei Aufnahme einer schweren Störung (Franz et al. 2000, Leichsenring et al. 2007).

Demgegenüber ist der Wert von 4,3, wie er bei den Göttinger Patienten vorlag, im Vergleich $\mathrm{zu}$ anderen stationär behandelten Psychotherapiepatienten vergleichsweise niedrig und findet sich laut Schepank (1995) eher im ambulanten Bereich. Warum sich die Patientengruppe in der Selbstbeurteilung hingegen deutlich schlechter einschätzte, kann mit den vorliegenden Daten nicht eindeutig erklärt werden. Denkbar ist, dass die Göttinger Therapeuten ihre Patienten systematisch als zu unbeeinträchtigt beurteilten. Zwar gilt die Reliabilität des BSS als hoch (Schepank 1995); aber auch Schauenburg et al. (2005) fanden in ihrer Untersuchung zur Bedeutung des Einzeltherapeuten im Rahmen einer stationären Therapie eine therapeutenabhängige Einschätzung des BSS. Es fällt auf, dass die Göttinger Patienten sich in der Selbstbeurteilung zum Entlassungszeitpunkt noch deutlich beeinträchtigter fühlten als die Hagener, von den Therapeuten aber, ähnlich wie zum Aufnahmezeitpunkt, als weniger beeinträchtigt eingeschätzt wurden. Dieser Befund könnte als weiterer Beleg für die Vermutung gelten, dass es sich hierbei um einen Therapeutenbias handelt.

In beiden Kliniken besserte sich die Beeinträchtigungsschwere signifikant im Verlauf; im Vergleich war der Effekt in der Hagener Population aber signifikant größer als in der Göttinger. Worin könnten die Gründe liegen? 
Zum einen war der Spielraum für eine Verbesserung der Beeinträchtigungsschwere bei den Göttinger Patienten geringer, da sie bereits mit vergleichsweise niedrigen Werten in die Behandlung einstiegen. Dies erklärt aber nicht, warum sich die Hagener Patienten so stark besserten, dass sie bei Entlassung nahezu den Göttinger Patienten entsprachen. Erklärungsansätze bieten sich bei differenzierter Betrachtung der Subkategorien.

Die Verbesserung der psychischen Befindlichkeit machte in beiden Patientengruppen den größten Anteil aus. Dies ist im Rahmen einer psychotherapeutischen Behandlung, die die Besserung vor allem des psychischen Befindens zum Ziel hat, nicht verwunderlich und entspricht anderen Studienergebnissen (Franz et al. 2000, Konzag et al. 2000, Leichsenring et al. 2007). Es zeigt sich somit, dass in beiden Kliniken dieser gewünschte Effekt erzielt wurde. Dennoch ist die Effektstärke in Hagen deutlich größer als in Göttingen.

Die Differenz der Beeinträchtigungsschwere wird in Hagen nicht nur durch die psychische, sondern im Vergleich zur Göttinger Gruppe sehr stark auch von der somatischen Dimension beeinflusst. Dies kann mit dem nicht unerheblichen Anteil von Suchtmittelgebrauch und einigen somatoformen Störungsbildern in der Hagener Patientengruppe in Zusammenhang stehen, bei denen sich die psychische Beeinträchtigung auch sehr stark körperlich auswirkt. Die Besserung des Zustandes wird dann bereits unter anderem durch eine längere Phase der Abstinenz von Suchtmitteln oder durch körper- und physiotherapeutische Maßnahmen erreicht.

Die auffälligste Differenz zeigt sich im Bereich der sozialkommunikativen Beeinträchtigung. Hier besserte sich die Hagener Patientengruppe mit großer Effektstärke, die Göttinger hingegen kaum. Dieser Befund ergänzt und bestärkt die bereits weiter oben (s. Kapitel 5.1, S.62) erörterte Annahme einer gezielteren Beeinflussung sozialkommunikativer Aspekte durch die psychoanalytisch-interaktionelle Gruppentherapie in der Hagener Gruppe. Diese These wird teilweise unterstützt durch den Vergleich mit der Literatur: Während sich in der Multizenterstudie von Franz et al. (2000), die sich eher unspezifisch auf psychodynamische Behandlungsverfahren bezog, die sozialkommunikative Dimension kaum veränderte, erzielte diese bei Leichsenring et al. (2007) in der psychoanalytisch-interaktionellen Therapie eine Veränderung mit hoher Effektstärke, die nahe an die Verbesserung der psychischen Dimension heranreichte. Bei Rabung et al. (2005) fand sich eine eindeutige Diskrepanz zwischen tiefenpsychologischer und psychoanalytisch-interaktioneller Gruppe hingegen nicht (bei allerdings deutlich unterschiedlicher Behandlungsdauer). In der Studie von Konzag et al. (2000) zu stationärer psychodynamischer Therapie zeigte sich interessanterweise in der Gruppe der „erfolglosen“ Patienten eine Verbesserung ausschließlich im sozialkommunikativen Bereich des BSS, was die Frage aufwirft, welche unspezifischen Faktoren die sozialkommunikativen Fähigkeiten verbessern.

Möglicherweise haben also sowohl spezifische Wirkfaktoren der psychoanalytischinteraktionellen Gruppentherapie als auch weitere unspezifische bzw. durch die Studie nicht erfasste Faktoren zur Differenz der Beeinträchtigungsschwere beigetragen.

In vergleichbaren Untersuchungen zur stationären Dialektisch-Behavioralen Therapie wurde der BSS nicht verwendet und kann somit nicht verglichen werden. 


\subsubsection{Stationserfahrungsbogen}

Die Ergebnisse der Stationserfahrungen konnten aufgrund methodischer Probleme lediglich deskriptiv in die Auswertung einbezogen werden, sie lieferten dennoch einen Eindruck zum Behandlungsverlauf auf den Stationen der unterschiedlichen Abteilungen aus der Sicht der Patienten.

Es zeigten sich keine wesentlichen Unterschiede zwischen den Kliniken, auch dann, wenn man sie mit den Werten der Vergleichsstichprobe von Sammet und Schauenburg (1999) in Beziehung setzt. Die Hagener Gruppe zeigte über alle Skalen etwas höhere Werte als die Göttinger, diese Differenz blieb über den fortschreitenden Behandlungsverlauf erhalten. Beide Kliniken unterschieden sich deutlich von der Vergleichsstichprobe in Bezug auf das Stationsklima; dieses wurde sowohl in Hagen als auch in Göttingen besser bewertet (vgl. Kapitel 4.4.6, Seite 55).

Die Interpretation der Ergebnisse ist allerdings mit aller Vorsicht zu treffen, da sich die Behandlungswochen (und die individuelle Bedeutung der Behandlungswoche) zwischen Hagen und Göttingen doch deutlich unterschieden. Die Göttinger Patienten bearbeiteten die Bögen zum Aufnahmezeitpunkt, die Hagener Patienten erst, nachdem feststand, dass sie an der Psychotherapiegruppe teilnahmen. Somit ist der Zeitpunkt t1 nicht identisch mit dem Zeitpunkt der Aufnahme. Des Weiteren ist die Therapiedauer in Hagen länger und variabler als in Göttingen; somit hat z. B. Behandlungswoche 4 für einen Patienten, der noch weitere drei Wochen behandelt wird, eine ganz andere Bedeutung als für einen Patienten, der in der folgenden Woche entlassen wird. Auf dieses Problem in der Interpretation weisen auch Sammet und Schauenburg (1999) hin und berechneten daher die Ergebnisse ihrer Vergleichsstichprobe aus den Durchschnittswerten ohne Berücksichtigung der Behandlungswoche. Eine detaillierte Betrachtung der Stationserfahrungen in Bezug zur Behandlungszeit wäre somit nur individuell für einzelne Patienten sinnvoll, war aber nicht Inhalt der Studie.

Der wesentliche Grund für den Einsatz des Stationserfahrungsbogens lag vielmehr in der Kontrolle der Einflussfaktoren auf die Behandlungsergebnisse, die bekanntermaßen im stationären Bereich vielfältig sind.

Sammet et al. (2004) versuchten die unterschiedlichen Einflussfaktoren im stationären Therapiesetting auf das Behandlungsergebnis näher einzugrenzen. Sie untersuchten eine Gruppe von stationären Psychotherapiepatienten im Hinblick auf die Symptomreduktion, die interpersonellen Probleme und die Zusammenhänge mit der einzeltherapeutischen Beziehung, der Beziehung zum gesamten Behandlungsteam und der Beziehungen innerhalb der Stationsgruppe (Kohäsion und Klima). Sie fanden dabei einen moderaten Einfluss der einzeltherapeutischen Beziehung und des Stationsklimas auf das Therapieergebnis. Dies sollte Anlass sein, insbesondere diesen beiden Items in der vorliegenden Studie Beachtung zu schenken.

Die Beziehung zum Einzeltherapeuten wurde in der Göttinger Gruppe gegenüber der Hagener über den gesamten Behandlungsverlauf nur gering schlechter eingeschätzt; bis auf den Beginn der Behandlung lagen die Werte aber zwischen der $25 \%$ und $75 \%$-Quartile der 
Vergleichsstichprobe. Der Einfluss dieses Faktors in der Untersuchung von Sammet et al. (2004) war allerdings nur moderat ausgeprägt. Bassler und Hoffmann (1993) sowie Bassler (1995) kamen ebenfalls zu dem Ergebnis, dass der Einfluss des Einzeltherapeuten im stationären Rahmen etwas an Bedeutung verliert. Kordy et al. (1990) demgegenüber weisen auf der Grundlage ihrer Ergebnisse der Einzeltherapie auch in stationärer Behandlung einen großen Stellenwert zu, ähnlich wie Schauenburg et al. (2005).

Ein messbar negativer Einfluss der einzeltherapeutischen Beziehung auf die Behandlungsergebnisse in Göttingen gegenüber Hagen kann hieraus nicht abgeleitet werden. Ein systematischer Einfluss der guten einzeltherapeutischen Beziehung in Hagen auf die Gruppentherapie kann allerdings ausgeschlossen werden, da Einzel- und Gruppentherapie (bis auf vereinzelte Ausnahmefälle) von unterschiedlichen Therapeuten durchgeführt wurden.

Das Stationsklima wurde sowohl in Hagen als auch in Göttingen als überdurchschnittlich gut bewertet und übertraf die $75 \%$ Quartile der Vergleichsgruppe. In Anlehnung an die Ergebnisse von Sammet et al. (2004) wäre dies ein potenzieller Einflussfaktor im Sinne eines positiven Behandlungsergebnisses. Auch Grabhorn et al. (2000) unterstreichen die Bedeutung der Gruppenkohäsion, bezogen auf den ,gesamten gruppentherapeutischen Raum“ und sehen diese ,als einen die therapeutische Wirksamkeit wesentlich beeinflussenden Faktor. Patienten, die sich in Therapie-Gruppen wohl und akzeptiert fühlen, erleben sich gleichzeitig eher als selbstsicher und handlungsbereit" (beide Zitate s. S. 317). Vermutlich hatte das gute Stationsklima also einen positiven Einfluss auf die Behandlungsergebnisse beider Kliniken, Unterschiede im Ergebnis können hierauf nicht zurückgeführt werden.

\subsection{Einschränkungen und Ausblick}

Diese Studie wurde bewusst als prospektive naturalistische, quasi-experimentelle Untersuchung durchgeführt, um die Wirkung von Psychotherapie, insbesondere psychoanalytisch-interaktioneller Gruppentherapie, im stationären klinischen Alltag zu untersuchen - nicht im universitären Forschungsbereich. Dieses Vorgehen wird von vielen Psychotherapieforschern als Alternative oder Ergänzung zu randomisierten kontrollierten Studien unter „Laborbedingungen“ favorisiert, vor allem um die komplexen Mechanismen psychotherapeutischer Behandlungen in der alltäglichen Praxis ausreichend zu berücksichtigen und möglichst hohe externe Validität zu gewährleisten (Leichsenring und Rüger 2004, Tschuschke 2005, Pfennig und Hölter 2011).

Die Absicht, gezielt nicht im universitären Bereich zu forschen, erhöht die Aussagefähigkeit für den allgemeinpsychiatrischen Alltag, schränkt die Ergebnisse aber gleichzeitig ein: Die personelle Kapazität war nicht für Forschungszwecke ausgelegt, und die Bedingungen zur Erhebung mussten in den Arbeitsalltag in einer nicht-universitären Versorgungsklinik integrierbar sein. Hierdurch ist zu erklären, dass nicht alle Daten, die zur Auswertung der Studie interessant gewesen wären, erhoben wurden.

Mit Hilfe der Patientengruppe in der Göttinger Klinik als Kontrollgruppe wurde versucht, den Einfluss der DBT als ebenfalls wirksames Psychotherapieverfahren herauszufiltern. Dennoch 
sind die beiden Kliniken nicht identisch in ihrer Struktur, und synergistische Effekte können damit nicht kontrolliert werden. Ebenso nicht zu kontrollierende Faktoren sind der Einfluss unterschiedlicher Einzeltherapeuten sowie weiterer Therapeuten, die an der Behandlung mitgewirkt haben. Auf den möglichen Therapeutenbias in der Beurteilung der Beeinträchtigungsschwere wurde bereits hingewiesen (s. Kapitel 5.4.4, S.73). Nicht unerwähnt bleiben sollte auch die Tendenz zu positiven Studienergebnissen bei starker Treue des Forschers zu ,seinem“ Therapieverfahren (vgl. die Untersuchung von Luborsky et al. (1999).

Als großer Nachteil muss die fehlende Erhebung weiterer soziodemografischer Daten, der drop-out-Raten und der Nichtteilnehmer gesehen werden. Möglicherweise wurde hierdurch eine Positivselektion begünstigt (Rentrop et al. 2010). Auch der Einfluss medikamentöser Behandlung wurde nicht berücksichtigt; um diesen allerdings genauer zu untersuchen, wäre die Population ohnehin zu klein gewesen.

Die Schwierigkeit, spezifische gruppentherapeutische Wirkfaktoren in einem multidimensionalen Behandlungssetting zu identifizieren, wurde ebenfalls bereits erläutert (vgl. Kapitel 1.6, Seite 20). In nachfolgenden Untersuchungen könnte dieser Umstand durch den Einsatz eines zusätzlichen Gruppenfragebogens, wie von Strauß et al. (1998) vorgeschlagen, eventuell besser berücksichtigt werden.

Die auffällige Differenz in Bezug auf die Besserung interpersoneller Probleme zwischen beiden untersuchten Populationen könnte außerdem für zukünftige Studien Anregung sein, die spezifische Wirkung psychoanalytisch-interaktioneller Gruppentherapie auch unter Berücksichtigung der verschiedenen interpersonellen Subgruppen zu evaluieren. 


\section{Zusammenfassung}

In der vorliegenden naturalistischen, quasi-experimentellen Studie wird die Wirksamkeit psychoanalytisch-interaktioneller Gruppentherapie in einer nicht-universitären allgemeinpsychiatrischen Klinik der Pflicht- und Regelversorgung untersucht.

Hierzu wurden 42 Patienten, die zwischen 2007 und 2009 in der Klinik für Psychiatrie und Psychotherapie der Katholischen Krankenhaus GmbH Hagen psychotherapeutisch mit psychoanalytisch-interaktioneller Gruppentherapie, ergänzt durch Elemente der DialektischBehavioralen Therapie, stationär behandelt wurden, bezüglich ihrer Symptomverbesserung untersucht. Als Kontrollgruppe dienten 40 Patienten, die im gleichen Zeitraum an einer stationären Behandlung auf einer psychotherapeutischen Station des Asklepios Fachklinikums Göttingen mit Dialektisch-Behavioraler Therapie, nicht jedoch an psychoanalytischinteraktioneller Gruppentherapie, teilnahmen. Testinstrumente waren Selbstbeurteilungsfragebögen zu Symptombelastung (SCL 90-R), interpersonellen Problemen (IIP) und der Lebenszufriedenheit (FLZ), ein Fremdbeurteilungsbogen zur Beeinträchtigungsschwere (BSS) im Prä-Post-Vergleich, sowie zur Kontrolle von Einflussfaktoren im Behandlungsverlauf der Stationserfahrungsbogen (SEB).

Es werden die soziodemografischen Daten zu Geschlecht, Alter und Schulbildung sowie klinische Daten zu Diagnosen nach ICD 10 und Behandlungsdauer auf signifikante Unterschiede überprüft. Für den Prä-Post-Vergleich werden Signifikanzen und Effektstärken berechnet. Der Einfluss der Behandlungsbedingung (Klinik) und des Untersuchungszeitpunktes (Prä-Post) sowie deren Interaktion werden varianzanalytisch in ihrem Einfluss auf die Testergebnisse überprüft. Die Ergebnisse des Stationserfahrungsbogens (SEB) werden deskriptiv behandelt.

Beide Populationen sind vergleichbar bezüglich Geschlechterverteilung und Schulbildung, sowie der Anzahl der Komorbiditäten. Die Hagener Gruppe weist gegenüber der Göttinger Gruppe ein signifikant höheres Lebensalter, eine größere Variabilität der Diagnosen mit geringerem Anteil an Persönlichkeitsstörungen und eine signifikant längere Behandlungsdauer auf.

Im Prä-Post-Vergleich lassen sich in beiden Kliniken signifikante Verbesserungen der Symptombelastung, Lebenszufriedenheit und Beeinträchtigungsschwere von mittlerer bis hoher Effektstärke nachweisen. Demgegenüber zeigt nur die Hagener Patientenpopulation signifikante Verbesserungen interpersoneller Probleme mit mittlerer Effektstärke. Im Zwischengruppenvergleich weist die Hagener Gruppe bezüglich Beeinträchtigungsschwere und interpersoneller Probleme signifikant bessere Ergebnisse auf als die Göttinger. Die Symptombelastung nimmt in der Hagener Gruppe zwar deutlicher ab als in der Göttinger, das Ergebnis verfehlt das Signifikanzniveau allerdings knapp. Bezüglich der Lebenszufriedenheit unterscheiden sich die Kliniken nicht signifikant. Keinen relevanten Unterschied zeigen die Stationserfahrungen der Patienten. 
Die Interpretation der Ergebnisse ist aufgrund methodischer Einschränkungen einer naturalistischen Untersuchung im nicht-universitären Bereich (z. B. fehlende Drop-out-Raten, begrenzte Vergleichbarkeit der Stichproben) nur mit Einschränkungen möglich. Die Ergebnisse können dennoch ein Hinweis darauf sein, dass die psychoanalytischinteraktionelle Gruppentherapie als psychotherapeutische Methode in einer allgemeinpsychiatrischen Klinik wirksam ist und dass möglicherweise eine spezifischere Beeinflussung interpersoneller Schwierigkeiten hierdurch bewirkt wird. Die genauere Validierung dieser Ergebnisse bleibt weiteren Forschungsvorhaben vorbehalten. 


\section{Literaturverzeichnis}

Allen J, Fonagy P (Hrsg.): Mentalisierungsgestützte Therapie, Klett-Cotta, Stuttgart 2009

Arfsten AJ, Hoffmann SO (1978): Stationäre psychoanalytische Psychotherapie als eigenständige Behandlungsform, Prax Psychother 23, 233-245

Auchincloss E (2002): The place of psychoanalytic treatments within psychiatry. Arch Gen Psychiatry 59, 501-502

Barley W, Buie S, Peterson E, Hollingsworth A, Griva M, Hickerson S, Lawson J, Bailey B (1993): Development of an inpatient cognitive-behavioral treatment program for borderline personality disorder. J Personal Disord 7, 232-240

Bassler M (1995): Prognosefaktoren für den Erfolg von psychoanalytisch fundierter stationärer Psychotherapie. Z Psychosom Med Psychoanal 41, 77-79

Bassler M, Hoffmann SO (1993): Die therapeutische Beziehung im Rahmen von stationärer Psychotherapie. Psychother Psychosom Med Psychol 43, 325-332

Bauer C, Machold C, Geyer M, Plöttner G (2005): Persönlichkeitsstörungen nach stationärer psychodynamischer Psychotherapie. Z Psychosom Med Psychother 51, 128-144

Berger M, Hohagen F (2012): Schulenorientierte versus störungsorientierte Psychotherapien in der Psychiatrie. Psychiatrie 9, 85-88

Berghaus A (2005): Psychoanalytisch-interaktionelle Methode, Affektspiegelung und Mentalisierung. Gruppenpsychother Gruppendynamik 41, 193-218

Beutel M, Höflich A, Kurth R, Brosig B, Gieler U, Leweke F, Milch W, Reimer C (2005): Stationäre Kurz- und Langzeitpsychotherapie - Indikationen, Ergebnisse, Prädiktoren. Z Psychosom Med Psychother 1ㅡ, 145-162

Bion W: Experiences in Groups. Tavistock, London 1961

Bion W, Rickman J (1943): Intra-group tensions in therapy. Lancet 1943, II, 678-681

Bohus M (2002): Borderline-Störung, (Fortschritte der Psychotherapie, Band 14), Hogrefe, Göttingen 2002

Bohus M, Haaf B (2001): Dialektisch-Behaviorale Therapie der Borderline-Störung im stationären Setting. Verhaltensther Psychosoz Prax $\underline{33}$, 619-642

Bohus M, Kröger C (2011): Psychopathologie und Psychotherapie der BorderlinePersönlichkeitsstörung. Nervenarzt $\underline{82}, 16-24$

Bohus M, Haaf B, Stiglmayr C, Pohl U, Boehme R, Linehan M (2000): Evaluation of inpatient dialectical-behavioral therapy for borderline personality disorder - a prospective study. Behav Res Ther $\underline{38}$, 875-887 
Bohus M, Haaf B, Simms T, Limberger M, Schmahl C, Unckel C, Lieb K, Linehan M (2004): Effectiveness of inpatient dialectical behavioral therapy. Behav Res Ther $\underline{42}$ 487-499

Bohus M, Herpertz S, Falkai P (2012): Modulare Psychotherapie - Rationale und Grundprinzipien. Psychiatrie $\underline{9}, 89-97$

Bolm T (2005): Psychoanalytisch orientierte Gruppenpsychotherapie in der Psychiatrie Praxis und Perspektiven des Göttinger Modells. Gruppenpsychother Gruppendynamik 41, 176-192

Borgart E, Meermann R (1999): Bedingungsfaktoren unterschiedlicher Behandlungsdauer bei Angststörungen im Rahmen stationärer Verhaltenstherapie. Psychother Psychosom Med Psychol 49, 109-113

Borgart E, Meermann R: Stationäre Verhaltenstherapie. Hans Huber, Bern 2004

Braner H, Hildenbrand G, Hanno I, Krüger C, Sturm J, Weidenhammer J (2007): Krankenhausbehandlung und/oder Rehabilitation - eine Kontroverse. Psychother Dialog $\underline{8}, 5$ 14

Burlingame G, Mac Kenzie K, Strauß B (2002): Zum aktuellen Stand der Gruppenpsychotherapieforschung: II. Effekte von Gruppenpsychotherapien als Bestandteil komplexer Behandlungsansätze. Gruppenpsychother Gruppendynamik $\underline{38}$, 5-32

Cohen J: Statistical power analysis for the behavioral sciences. Lawrence Erlbaum, Hillsdale 1988

Crits-Christoph P, Connolly Gibbons MB, Narducci J, Schamberger M (2005): Interpersonal problems and the outcome of interpersonally oriented psychodynamic treatment of GAD. Psychotherapy $\underline{42}, 211-224$

Davies-Osterkamp S, Jung K, Ott J (1992): Therapeutische Faktoren in der psychoanalytischinteraktionellen und tiefenpsychologisch fundierten Gruppentherapie: Eine empirische Untersuchung. Psychother Psychosom Med Psychol 42, 102-109

DeNeve K, Cooper H (1998): The happy personality: A meta-analysis of 137 personality traits and subjective well-being. Psychol Bull 124, 197-229

Derogatis LR: SCL-90-R, administration, scoring \& procedures manual-I for the R(evised) version and other instruments of the psychopathology rating scale series. Johns Hopkins University, School of Medicine, Baltimore, USA 1977

Deutscher Bundestag 7. Wahlperiode: Bericht über die Lage der Psychiatrie in der Bundesrepublik Deutschland. Psychiatrieenquete. Bundesdrucksache 7/4200, Bonn 1975

Dilling $\mathrm{H}$, Mombour $\mathrm{W}$, Schmidt $\mathrm{MH}$, Schulte-Markwort (Hrsg.): Internationale Klassifikation psychischer Störungen ICD-10 Kapitel V(F). 4. Auflage, Hans Huber, Bern 2006 
Dinger U, Schauenburg H (2010): Effects of individual cohesion and patient interpersonal style on outcome in psychodynamically oriented inpatient group psychotherapy. Psychother Res 20, 22-29

Fahrenberg J, Myrtek M, Wilk D, Kreutel K (1986): Multimodale Erfassung der Lebenszufriedenheit: Eine Untersuchung an Herz-Kreislauf-Patienten. Psychother Psychosom Med Psychol 36, 347-354

Fahrenberg J, Myrtek M, Schumacher J, Brähler E: Fragebogen zur Lebenszufriedenheit (FLZ). Hogrefe, Göttingen 2000

Falkai P, Herpertz S (2012): Entwurf für ein neues Weiterbildungscurriculum für Psychotherapie in der Psychiatrie. Psychiatrie $\underline{9}$, 99-104

Fonagy P, Bateman A (2006): Mechanisms of change in mentalization-based treatment of BPD. J Clin Psychol 62, 411-430

Franke G: SCL-90-R. Die Symptom-Checkliste von L. R. Derogatis - Deutsche VersionManual. 2. Auflage, Beltz-Test GmbH, Göttingen 2002

Franke G, Hoffmann T, Frommer J (2005): Entspricht die Symptombesserung vier Wochen nach Behandlungsbeginn dem Erfolg in der Ein-Jahres-Katamnese? Z Psychosom Med Psychother 51, 360-372

Franz M, Janssen P, Lensche H, Schmidtke V, Tetzlaff M, Martin K, Wöller W, Hartkamp N, Schneider G, Heuft G (2000): Effekte stationärer psychoanalytisch orientierter Psychotherapie - eine Multizenterstudie. Z Psychosom Med Psychother 46, 242-258

Freud S (1937): Die endliche und die unendliche Analyse (Gesammelte Werke Band XVI, 2. Auflage), Imago Publishing Co., London 1961, 57-99

Freyberger H, Kächele J: Psychodynamische Psychiatrie- von Kretschmer zu Gabbard (Vorwort zur deutschen Ausgabe), in: Gabbard G: Psychodynamische Psychiatrie, Psychosozial-Verlag Gießen 2010, ix-xiv

Freyberger H, Spitzer C: Gruppenpsychotherapie in der Psychiatrie, in: Gruppenpsychotherapie, hrsg. von Strauß B, Mattke D, Springer, Berlin 2012, 417-426

Fürstenau P (1977): Die beiden Dimensionen des psychoanalytischen Umgangs mit strukturell ich-gestörten Patienten. Psyche $\underline{31}$, 197-207

Gabbard G: Psychodynamische Psychiatrie. Deutsche Erstveröffentlichung; PsychosozialVerlag, Gießen 2010

Gabbard G, Gunderson J, Fonagy P (2002): The place of psychoanalytic treatments within psychiatry. Arch Gen Psychiatry $\underline{59}$, 505-510

Gantner A: Psychotherapeutische Behandlung von Ecstasy- und Partydrogenkonsumenten, in: Ecstasy - Wirkungen, Risiken, Interventionen. Ein Leitfaden für die Praxis, hrsg. von Thomasius R, Enke Verlag, Stuttgart 1999 
Geiser F, Imbierowicz K, Conrad R, Wermter F, Liedtke R (2004): Verbessert die zusätzliche Durchführung einer verhaltenstherapeutischen Intervention die Ergebnisse stationärer psychodynamischer Therapie bei Panikstörung/Agoraphobie? Z Psychosom Med Psychother 50, 53-69

Grabhorn R, Kaufhold J, Burkhardt M, Kernhof K, Overbeck G (2000): Die Bedeutung differentiellen Gruppenerlebens während einer stationären Psychotherapie. Gruppenpsychother Gruppendynamik 36, 317-332

Grabhorn R, Kaufhold J, Burkhardt M, Kernhof K, Overbeck G, Gitzinger I (2002): Gruppenkohäsion und Abwehrverhalten im Verlauf stationärer Gruppentherapien. Psychother Psychosom Med Psychol 52, 275-281

Günther U, Lindner J (1999): Die psychoanalytisch begründete Kurzgruppenpsychotherapie unter rehabilitationsbezogenem Fokus. Gruppenpsychother Gruppendynamik 35, 203-221

Gunzelmann T, Schumacher J, Strauß B, Brähler E (2000): Interpersonale Probleme älterer Menschen. Psychotherapeut $\underline{45}, 153-164$

Harley R, Sprich S, Safren S, Jacobo M, Fava M (2008): Adaptation of dialectical behavior therapy skills training group for treatment-resistant depression. J Nerv Ment Dis $\underline{196}$, 136-143

Harned M, Chapman A, Dexter-Mazza E, Murray A, Comtois K, Linehan M (2008): Treating co-occurring axis I disorders in recurrently suicidal women with borderline personality disorder: A 2-year randomized trial of dialectical behavior therapy versus community treatment by experts. J Consult Clin Psychol 트, 1068-1075

Haubl R: Der institutionelle und organisatorische Kontext von Gruppen am Beispiel stationärer Gruppenpsychotherapie, in: Gruppenpsychotherapie, hrsg. von Strauß B, Mattke D, Springer, Berlin 2012, 99-107

Heigl-Evers A, Heigl F (1983): Das interaktionelle Prinzip in der Einzel- und Gruppenpsychotherapie. Z Psychosom Med Psychoanal 29, 1-14

Heigl-Evers A, Henneberg-Mönch U (1985): Psychoanalytisch-interaktionelle Therapie bei

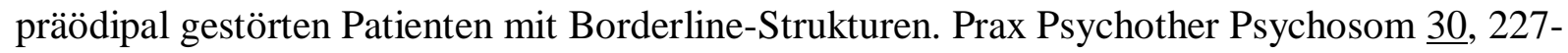
235

Heigl-Evers A, Streeck U (1985): Psychoanalytisch-interaktionelle Therapie. Psychother Psychosom Med Psychol 35, 176-182

Heigl-Evers A, Ott J (Hrsg.): Die psychoanalytisch-interaktionelle Methode, 4. Auflage, Vandenhoeck \& Ruprecht, Göttingen 2002

Herbold W, Sachsse U (2008): Schritt für Schritt - Differenzialindikation und spezialisierte stationäre Psychotherapie in einer psychiatrisch-psychotherapeutischen Fachklinik der Regionalversorgung. Fortschr Neurol Psychiatr 두, 155-159 
Hoffmann SO, Brodthage $\mathrm{H}$, Trimborn W, Stemmer T: Stationäre psychoanalytische Psychotherapie als eigenständige Behandlungsform, in: Psychotherapie im Krankenhaus, hrsg. von Heigl F, Neun H, Vandenhoek\&Ruprecht, Göttingen 1981, 35-40

Hoffmann SO, Egle UT, Bassler M, Nickel R, Petrak F, Porsch U (1998): Psychotherapeutische Kombinationsbehandlung. Psychotherapeut $\underline{43}$, 282-287

Hoglend P (1993): Personality disorders and long-term outcome after brief psychodynamic therapy. J Personal Disord $\underline{7}, 168-181$

Horowitz LM, Rosenberg SE, Baer BA, Ureno G, Villasenor V (1988): Inventory of Interpersonal Problems: Psychometric properties and clinical applications. J Consult Clin Psychol 56, 885-892

Horowitz LM, Strauß B, Kordy H : Inventar zur Erfassung interpersonaler Probleme Deutsche Version - 2. Auflage, Beltz-Test GmbH, Göttingen 2000

Huber D, Henrich G, Klug G (2007): The inventory of interpersonal problems (IIP): Sensitivity to change. Psychother Res $\underline{17}, 474-481$

Janssen P: Psychoanalytische Therapie in der Klinik. Klett-Cotta, Stuttgart 1987

Janssen P (2004): Berufsgruppen- und methodenintegrierende Teamarbeit in der stationären psychodynamischen Psychotherapie. Psychotherapeut 49, 217-226

Janssen P, Martin K, Tress W, Zaudig M (1998): Struktur und Methodik der stationären Psychotherapie aus psychoanalytischer und verhaltenstherapeutischer Sicht. Psychotherapeut $\underline{43}, 265-276$

Junge A, Ahrens S (1996): Stationäre psychosomatische Behandlung - Patientenmerkmale und Behandlungserfolg. Psychother Psychosom Med Psychol 46, 430-437

Kernberg O (2002): Psychoanalytic contributions to psychiatry. Arch Gen Psychiatry 59, 497498

Kirchmann H, Mestel R, Schreiber-Willnow K, Mattke D, Seidler KP, Daudert E, Nickel R, Papenhausen R, Eckert J, Strauss B (2009): Associations among attachment characteristics, patients' assessment of therapeutic factors, and treatment outcome following inpatient psychodynamic group psychotherapy. Psychother Res $\underline{19}$, 234-248

Kirchmann H, Schreiber-Willnow K, Seidler KP, Strauss B (2011): SCL-90-R symptom profile clusters among inpatients undergoing psychodynamic group psychotherapy: cluster stability, associations with clinical characteristics and treatment outcome. Psychopathology $\underline{44}, 71-82$

Köllner V, Vauth R (2007): Stationäre Verhaltenstherapie. Psychother Dialog, $\underline{8}, 21-27$

König K, Lindner WV: Psychoanalytische Gruppentherapie, 2. Auflage, Vandenhoeck \& Ruprecht, Göttingen 1992 
König O: Gruppendynamische Grundlagen, in: Gruppenpsychotherapie, hrsg. von Strauß B, Mattke D, Springer, Berlin 2012, 21-36

Kösters M, Strauß B (2007): Wirksamkeit stationärer Gruppenpsychotherapie - Eine kritische Betrachtung deutschsprachiger Studien. Gruppenpsychother Gruppendynamik $\underline{43}$, 181-200

Konzag TA, Fikentscher E (1998): Stationäre Psychotherapie. Psychotherapeut $\underline{43}$, 369-376

Konzag TA, Fikentscher E, Bandemer-Greulich U (2000): Vernetzte Evaluierung von Prozess- und Ergebnisqualität in der stationären Psychotherapie. Psychother Psychosom Med Psychol 50, 376-383

Koons C, Robins C, Tweed J, Lynch T, Gonzalez A, Morse J, Bishop G, Butterfield M, Bastian L (2001): Efficacy of dialectical behavior therapy in women veterans with borderline personality disorder. Behav Ther $\underline{32}, 371-390$

Kordy H, von Rad M, Senf W (1990): Therapeutische Faktoren bei stationärer Psychotherapie - Die Sicht des Patienten. Psychother Psychosom Med Psychol 40, 380-387

Kosarz P, Schwarz D: Konzepte, Entwicklungen und Rahmenbedingungen verhaltenstherapeutischer Kliniken, in: Die stationäre Psychotherapie und ihr Rahmen, hrsg. von Schepank H, Tress W, Springer-Verlag, Berlin 1988, 151-156

Kraft S, Percevic R, Puschner B, Kordy H (2003): Änderungsmuster in der Psychotherapie: Abhängigkeiten im Verlauf psychischer, sozialer und körperlicher Gesundung. Psychotherapie $\underline{8}, 218-224$

Kröger C, Schweiger U, Sipos V, Arnold R, Kahl K, Schunert T, Rudolf S, Reinecker H (2006): Effectiveness of dialectical behaviour therapy for borderline personality disorder in an inpatient setting. Behav Res Ther 44, 1211-1217

Leary T : Interpersonal diagnosis of personality. Ronald Press, New York 1957

Leichsenring F, Leibing E (2003): Wie wirksam sind psychoanalytisch orientierte Therapie und Verhaltenstherapie bei Persönlichkeitsstörungen? Forum Psychoanal 19, 378-385

Leichsenring F, Rüger U (2004): Psychotherapeutische Behandlungsverfahren auf dem Prüfstand der Evidence Based Medicine (EBM). Z Psychosom Med Psychother 50, 203-217

Leichsenring F, Dümpelmann M, Berger J, Jaeger U, Rabung S (2005): Ergebnisse stationärer psychiatrischer und psychotherapeutischer Behandlung von schizophrenen, schizoaffektiven und anderen psychotischen Störungen. Z Psychosom Med Psychother 51, 23-37

Leichsenring F, Masuhr O, Jaeger U, Dally A, Streeck U (2007): Zur Wirksamkeit psychoanalytisch-interaktioneller Therapie am Beispiel der Borderline-Pesönlichkeitsstörung: Daten aus der klinischen Praxis. Z Psychosom Med Psychother 53, 129-143

Liedtke R, Geiser F (2001): Veränderungen interpersonaler Probleme während und zwei Jahre nach stationärer Psychotherapie. Gruppenpsychother Gruppendynamik $\underline{37}$, 214-228 
Linden M, Baudisch F, Popien C, Golombek J (2006): Das ökologisch-therapeutische Milieu in der stationären Behandlung. Psychother Psychosom Med Psychol 56, 390-396

Linehan M.: Dialektisch-Behaviorale Therapie der Borderline-Persönlichkeitsstörung CIPMedien, München 1996a

Linehan M: Trainingsmanual zur Dialektisch-Behavioralen Therapie der BorderlinePersönlichkeitsstörung. CIP-Medien, München 1996b

Linehan M, Armstrong H, Suarez A, Allmon D, Heard H (1991): Cognitive-behavioral treatment of chronically parasuicidal borderline patients. Arch Gen Psychiatry $\underline{48}, 1060-1064$

Linehan M, Tutek D, Heard H, Armstrong H (1994): Interpersonal outcome of cognitive behavioral treatment for chronically suicidal borderline patients. Am J Psychiatry 151, 17711776

Linehan M, Schmidt H, Dimeff L, Craft J, Kanter J, Comtois K (1999): Dialectical behavior therapy for patients with borderline personality disorder and drug-dependence. Am J Addict $\underline{8}, 279-292$

Linehan M, Comtois K, Murray A, Brown M, Gallop R, Heard H, Korslund K, Tutek D, Reynolds S, Lindenboim N (2006): Two-year randomized controlled trial and follow-up of dialectical behavior therapy vs. therapy by experts for suicidal behaviors and borderline personality disorder. Arch Gen Psychiatry 63, 757-766

Luborsky L, Diguer L, Seligman D, Rosenthal R, Krause E, Johnson S, Halperin G, Bishop M, Berman J, Schweizer E (1999): The researcher's own therapy allegiances: A "wild card" in comparisons of treatment efficacy. Clin Psychol $\underline{6}, 95-106$

Lynch R, Trost W, Salsman N, Linehan M (2007): Dialectical behavior therapy for borderline personality disorder. Annu Rev Clin Psychol $\underline{3}$, 181-205

Main T (1946): The hospital as a therapeutic institution. Bull Menninger Clin $\underline{10}$, 66-70

Marziali E, Munroe-Blum H, McCleary L (1997): The contribution of group cohesion and group alliance to the outcome of group psychotherapy. Int J Group Psychother 47, 475-497:

Mentzos S: Lehrbuch der Psychodynamik. 3. Auflage; Vandenhoek \& Ruprecht, Göttingen 2009

Möhlen K, Heising G (1980): Integrative stationäre Psychotherapie. Gruppenpsychother Gruppendynamik $\underline{15}, 16-31$

Mohr D, Beutler L, Engle D, Shoham-Salomon V, Bergan J, Kaszniak A, Yost E (1990): Identification of patients at risk for nonresponse and negative outcome in psychotherapy. $\mathbf{J}$ Consult Clin Psychol $\underline{58}$, 622-628

Newsom J, Schulz R (1996): Social support as a mediator in the relation between functional status and quality of life in older adults. Psychol Aging 11, 34-44 
Nickel R, Egle U: Therapie somatoformer Schmerzstörungen. Schattauer, Stuttgart 1999

Nosper M (1999): Der Erfolg psychosomatischer Rehabilitation in Abhängigkeit von der Behandlungsdauer. Psychother Psychosom Med Psychol 49, 354-360

Orlinsky D, Grawe K, Parks B: Process and outcome in psychotherapy; in: Handbook of psychotherapy and behavior change, hrsg. von Bergin A, Garfield S, 4. Auflage, John Wiley and Sons, New York 1994, 270-378

Ott J (2001): Die psychoanalytisch-interaktionelle Gruppentherapie - ein Behandlungsangebot für Patienten mit strukturellen Störungen. Psychother Dialog 2, 51-58

Pfammatter M, Junghan U (2012): Integrierte psychotherapeutische Behandlung von schwer psychisch Kranken. Nervenarzt $\underline{83}, 861-868$

Pfennig A, Hölter G (2011): Evidence-based Medicine ist der Goldstandard der Leitlinienentwicklung. Psychiatr Prax $\underline{38}, 218-220$

Rabung S, Leichsenring F, Jaeger U, Streeck U (2005): Behandlungsergebnisse psychoanalytisch-interaktioneller und analytisch orientierter stationärer Gruppentherapie. Gruppenpsychother Gruppendynamik 41, 130-152

Rentrop M, Martius P, Bäuml J, Buchheim P, Döring S, Hörz S (2010): Patients with borderline personality disorder not participating in an RCT: Are they different? Psychopathology $\underline{43}, 369-372$

Riedel WP (1991): Einige Patientenmerkmale als Determinanten des Therapieerfolgs in der stationären Psychotherapie. Z Psychosom Med Psychoanal 37, 14-30

Riedel-Heller S, Becker T (2012): S3-Leitlinie Psychosoziale Therapien bei schweren

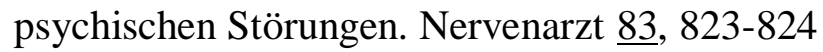

Rudolf G: Strukturbezogene Psychotherapie. Schattauer, Stuttgart 2004

Rudolf G, Jakobsen T, Micka R, Schumann E (2004): Störungsbezogene Ergebnisse psychodynamisch-stationärer Psychotherapie. Z Psychosom Med Psychother 50, 37-52

Sack M, Lempa W, Lamprecht F, Schmid-Ott G (2003): Therapieziele und Behandlungserfolg: Ergebnisse einer Katamnese ein Jahr nach stationärer psychosomatischer Therapie. Z Psychosom Med Psychother 49, 63-73

Salzer S, Streeck U, Jaeger U, Masuhr O, Warwas J, Leichsenring F, Leibing E (2010): Der Zusammenhang von interpersonalen Subtypen und der Veränderung interpersonaler Probleme am Beispiel stationärer Psychotherapiepatienten. Z Psychosom Med Psychother 56, 191-206

Sammet I, Schauenburg H: Stations-Erfahrungsbogen. Fragebogen zur Erfassung des Verlaufs stationärer Psychotherapie. Beltz-Test Test GmbH, Göttingen 1999

Sammet I, Staats H, Schauenburg H (2004): Beziehungserleben und Therapieergebnis in stationärer Psychotherapie. Z Psychosom Med Psychother 50, 376-393 
Schauenburg H, Dinger U, Strack M (2005): Zur Bedeutung der Einzeltherapeuten für das Therapieergebnis in der stationären Psychotherapie - eine Pilotstudie. Psychother Psychosom Med Psychol 55, 339-346

Schellenberg K, Niebecker M, Mattke D, Hofmann A, Schreiber-Willnow K, Hussy W (2004): Therapie-Evaluation bei traumatisierten Patienten mit dissoziativen Symptomen in einer stationären psychosomatischen Behandlung. Gruppenpsychother Gruppendynamik $\underline{40}$, 248-276

Schepank H: Die stationäre Psychotherapie in der Bundesrepublik Deutschland: Soziokulturelle Determinanten, Entwicklungsstufen, Ist-Zustand, internationaler Vergleich, Rahmenbedingungen, in: Die stationäre Psychotherapie und ihr Rahmen, hrsg. von Schepank H, Tress W, Springer-Verlag, Berlin 1988, 13-38

Schepank H: Der Beeinträchtigungs-Schwere-Score. Ein Instrument zur Bestimmung der Schwere einer psychogenen Erkrankung. Beltz-Test Test GmbH, Göttingen 1995

Schulz H, Barghaan D, Harfst T, Dirmaier J, Watzke B, Koch U (2006): Versorgungsforschung in der psychosozialen Medizin. Bundesgesundheitsblatt Gesundheitsforschung Gesundheitsschutz, 쏘, 175-187

Senf W: Theorie der stationären Psychotherapie; in: Praxis der stationären Psychotherapie, hrsg. von Becker H, Senf W, Thieme Verlag Stuttgart 1988, 2-34

Senf W (2007): „Ich habe etwas gefunden, was mir entsprach“. Psychother Dialog $\underline{8}$, 3-4

Silveira E, Ebrahim S (1998): Social determinants of psychiatric morbidity and well-being in immigrant elders and whites in east London. Int J Geriatr Psychiatry 13, 801-812

Simmel E (1928): Die psychoanalytische Behandlung in der Klinik. Int J Psychoanal 14, 352370

Soler J, Pascual J, Tiana T, Cebrià A, Barrachina J, Campins M, Gich I, Alvarez E, Pérez V (2009): Dialectical behavior therapy skills training compared to standard group therapy in borderline personality disorder: A 3-month randomized controlled clinical trial. Behav Res Ther $\underline{47}, 353-358$

Springer T, Silk K (1996): A review of inpatient group therapy for borderline personality disorder. Harv Rev Psychiatry $\underline{3}, 268-278$

Stiglmayr C, Schehr K, Bohus M (2002): Fertigkeiten-Training im Rahmen der DialektischBehavioralen Therapie für Borderline-Persönlichkeitsstörungen. Persönlichkeitsstörungen $\underline{2}$, 126-134

Strauß B, Burgmeier-Lohse M (1994): Evaluation einer stationären Langzeitgruppenpsychotherapie - Ein Beitrag zur differentiellen Psychotherapieforschung im stationären Feld. Psychother Psychosom Med Psychol 44, 184-192 
Strauß B, Kriebel R, Mattke D (1998): Probleme der Qualitätssicherung in der stationären Gruppenpsychotherapie. Psychotherapeut $\underline{43}$, 18-25

Streeck U (1983): Abweichungen vom ,fiktiven Normal-Ich“: Zum Dilemma der Diagnostik struktureller Ich-Störungen. Z Psychosom Med Psychoanal 29: 334-349

Streeck U (1991): Klinische Psychotherapie als Fokalbehandlung. Z Psychosom Med Psychoanal $\underline{37}, 3-13$

Streeck U (1998): Persönlichkeitsstörungen und Interaktion. Psychotherapeut $\underline{43}, 157-163$

Streeck U: Über eine Art, in therapeutischer Interaktion zu reden. Zum antwortenden Interventionsmodus in der psychoanalytisch-interaktionellen Therapie, in: Die psychoanalytisch-interaktionelle Methode, hrsg. von Heigl-Evers A, Ott J, 4. Auflage, Vandenhoeck\&Ruprecht, Göttingen 2002a, 112-132

Streeck U (2002b): Handeln im Angesicht des Anderen. Über nicht-sprachliche Kommunikation in therapeutischen Dialogen. Psyche $\underline{56}$, 247-274

Streeck U (2002c): Gestörte Verhältnisse - zur psychoanalytisch-interaktionellen Gruppentherapie von Patienten mit schweren Persönlichkeitsstörungen. Persönlichkeitsstörungen $\underline{6}, 109-125$

Streeck U: Psychotherapie komplexer Persönlichkeitsstörungen. Klett-Cotta, Stuttgart 2007

Strupp H (2001): Implications of the Empirically Supported Treatment movement for psychoanalysis. Psychoanal Dialogues $\underline{11}$, 605-619

Sullivan HS: The interpersonal theory of psychiatry. Norton, New York 1953

Tschuschke V (2004): Gruppenpsychotherapie. Psychotherapeut 49, 101-109

Tschuschke V (2005): Die Psychotherapie in Zeiten evidenzbasierter Medizin. Psychotherapeutenjournal 2, 106-115

Tschuschke V, Catina A, Beckh T, Salvini D (1992): Wirkfaktoren in stationärer analytischer Gruppenpsychotherapie. Psychother Psychosom Med Psychol 42, 91-101

Turner R (2000): Naturalistic evaluation of dialectical behavior therapy-oriented treatment for borderline personality disorder. Cogn Behav Pract $\underline{7}$, 413-419

Wagner C: Zur Verweildauer in stationärer Psychotherapie. Eine empirisch-analytische Untersuchung von Einflußfaktoren. Med. Diss. Göttingen 1996

Wallerstein R (2002): Psychoanalytic treatments within psychiatry: An expanded view. Arch Gen Psychiatry 59, 499-500

Weizsäcker v.V (1925): Randbemerkungen über Aufgabe und Begriff der Nervenheilkunde. Dtsch Z Nervenheilkd $\underline{87}, 1-27$

Winnicott D: Vom Spiel zur Kreativität. Klett-Cotta, Stuttgart 1987 
Wolf A, Hertel G, Schreiber-Willnow K, Heuft G, Vandieken R (2011): Outcome und Katamnese stationärer psychosomatischer Krankenhaus-Behandlungen über 60-Jähriger. Z Psychosom Med Psychother 57, 251-260

Wuchner M, Eckert J, Biermann-Ratjen EM (1993): Vergleich von Diagnosegruppen und Klientelen verschiedener Kliniken. Gruppenpsychother Gruppendynamik 29, 198-214

Yalom I: Theorie und Praxis der Gruppenpsychotherapie. Pfeiffer-Verlag, München 1996

Zielke M, Dehmlow A, Wülbeck B, Limbacher K (1997): Einflußfaktoren auf die Behandlungsdauer bei psychosomatischen Erkrankungen in der stationären Verhaltenstherapie. Prax Klin Verhaltensmed Rehabil 10, 22-56 


\section{Danksagung}

Mein herzlicher Dank gilt Herrn Prof. Dr. med. U. Streeck, M.A., insbesondere für seine Ermutigung zu dieser Arbeit und seine konstruktive, engagierte und freundliche Unterstützung.

Herrn Prof. Dr. rer. nat. F. Leichsenring danke ich für die Anregungen zum Studiendesign, meinem Chefarzt Herrn Dr. med. N. Grünherz und Herrn Dr. med. W. Herbold für ihr Interesse und die Möglichkeit, die Untersuchung im klinischen Alltag durchzuführen.

Besonderer Dank gilt außerdem Herrn Dipl. Psych. U. Jaeger und Herrn Dipl. Psych. O. Masuhr für ihre kompetente und geduldige Unterstützung in der statistischen Auswertung.

Nicht zuletzt danke ich den Behandlungsteams der betroffenen Stationen und den Patienten, ohne deren Mitarbeit diese Arbeit nicht möglich gewesen wäre. 